\title{
$\underset{1}{+1}$

Framework for Integrated Complex Urban Systems (FICUS)

\section{Quantifying Uncertainty in Population Weighting of Twitter Analyses for Urban Risk Assessment}

Elizabeth G. Bastian, Natalie R. Myers, Charles R. Ehlschlaeger, and Jeffrey A. Burkhalter

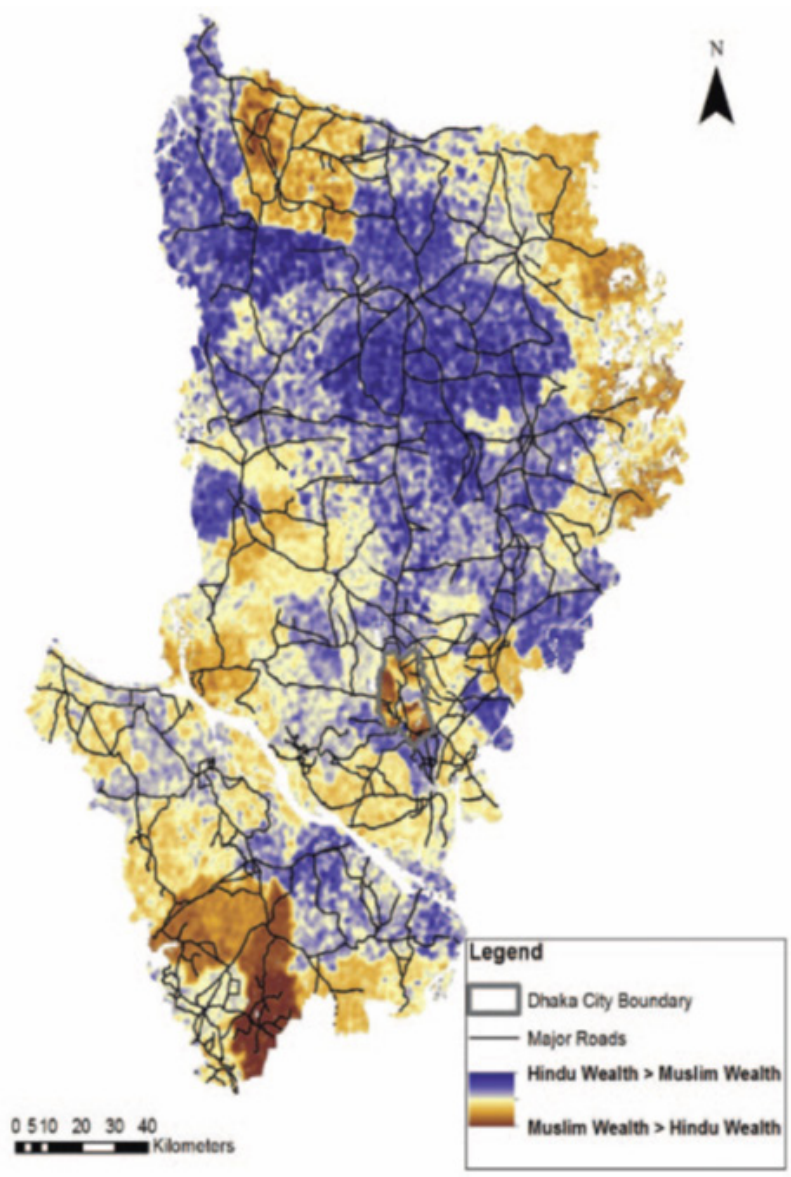


The U.S. Army Engineer Research and Development Center (ERDC) solves the nation's toughest engineering and environmental challenges. ERDC develops innovative solutions in civil and military engineering, geospatial sciences, water resources, and environmental sciences for the Army, the Department of Defense, civilian agencies, and our nation's public good. Find out more at www.erdc.usace.army.mil.

To search for other technical reports published by ERDC, visit the ERDC online library at http://acwc.sdp.sirsi.net/client/default. 


\section{Quantifying Uncertainty in Population Weighting of Twitter Analyses for Urban Risk Assessment}

Natalie R. Myers, Charles R. Ehlschlaeger, and Jeffrey A. Burkhalter

Construction Engineering Research Laboratory

U.S. Army Engineer Research and Development Center

2902 Newmark Drive

Champaign, IL 61822

Elizabeth G. Bastian

Center for Global Studies

Department of Urban and Regional Planning

University of Illinois, Urbana, IL 61801

Final Technical Report (TR)

Approved for public release; distribution is unlimited.

Prepared for Assistant Secretary of the Army for Acquisition, Logistics, and Technology 103 Army Pentagon

Washington, DC 20314-1000

Under Program Element 622784T41, Project P2 458304, "Framework for Integrating the Complexity of Urban Systems (FICUS)" 


\section{Abstract}

Twitter has increasingly been used to study various research topics such as election predictions, disease spread, etc. However, social media platforms do not saturate the entire population in a study area, especially in emerging nations, only representing more affluent subpopulations. The U.S. Army Engineer Research and Development Center, Construction Engineering Research Laboratory (ERDC-CERL), as part of a project entitled Framework for the Integration of Complex Urban Systems (FICUS), is quantifying the utility of demographic information to inform neighborhood-scale social media models. Using the example topic of infrastructure, an open-source model was constructed to collect Twitter data from the metropolitan Philippines area of Manila, geotag tweets to neighborhood grid cells based on language analysis, and produce a sentiment topic map. ERDC's social media analysis tools incorporate quantifiable uncertainties with specific on-the-ground reporting techniques. By using the Humanitarian Crisis (HC) framework developed by PACOM (another FICUS product) as a model, a framework quantifying the likelihood of being a regular social media user was created to implement a data-driven, bottom-up framework construction nested within a knowledge-based established framework. This framework, and any other produced by the FICUS team serve as case studies for augmenting the military operational environment with quantifiable reduced uncertainties.

DISCLAIMER: The contents of this report are not to be used for advertising, publication, or promotional purposes. Citation of trade names does not constitute an official endorsement or approval of the use of such commercial products. All product names and trademarks cited are the property of their respective owners. The findings of this report are not to be construed as an official Department of the Army position unless so designated by other authorized documents.

DESTROY THIS REPORT WHEN NO LONGER NEEDED. DO NOT RETURN IT TO THE ORIGINATOR. 


\section{Contents}

Abstract...................................................................................................................................... if

Figures and Tables...................................................................................................................................iv

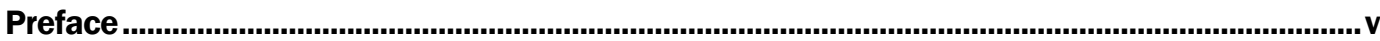

1 Introduction........................................................................................................................ 1

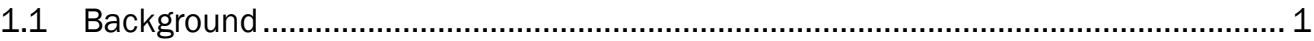

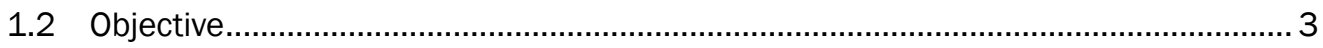

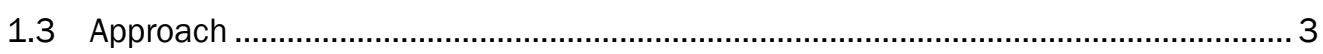

2 FICUS Demographic Models ................................................................................................... 4

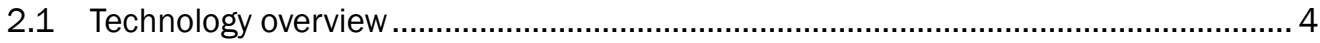

2.2 Initial humanitarian crisis framework .................................................................. 7

2.3 Social media use in the Philippines........................................................................ 9

3 Developing the FICUS Twitter Tool ...........................................................................................11

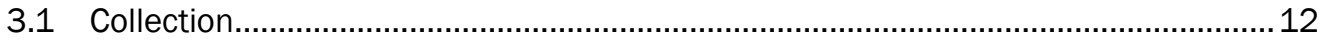

3.2 Geotagging based on language modeling ........................................................ 13

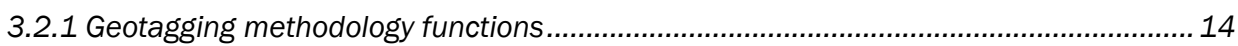

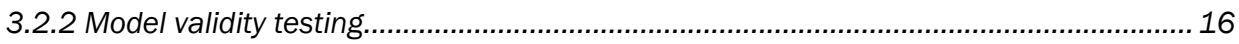

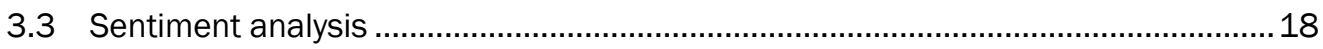

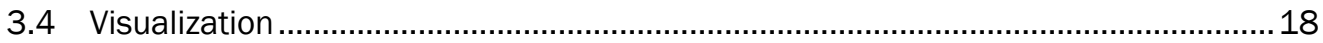

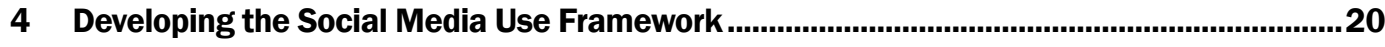

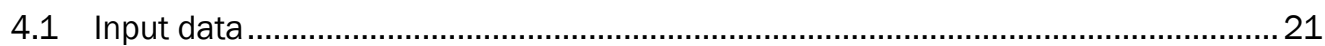

4.2 Risk value ............................................................................................................ 21

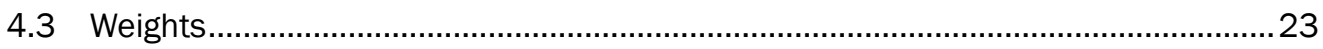

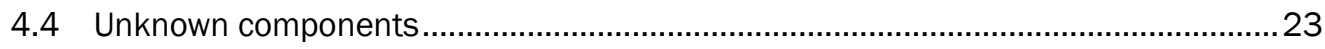

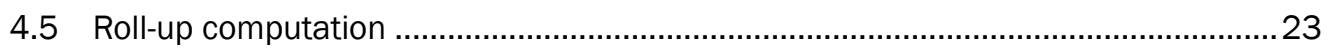

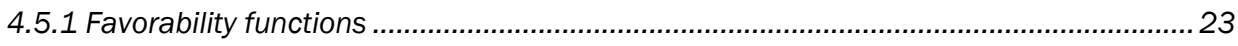

4.5.2 Quantifying errors and uncertainty ................................................................... 25

5 Augmenting Twitter Sentiment Maps with Social Media Framework Maps.........................27

6 Conclusion..........................................................................................................................29

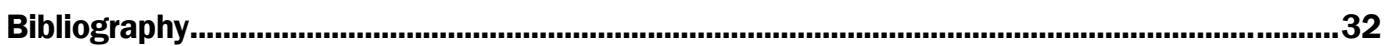

Appendix A: Social Media Use (Twitter) Framework Technical Documentation ........................36

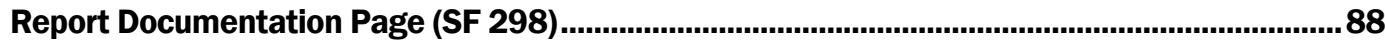




\section{Figures and Tables}

\section{Figures}

$1 \quad$ Estimated average wealth inequity between Muslims and Hindus near

Dhaka, Bangladesh ..................................................................................................... 5

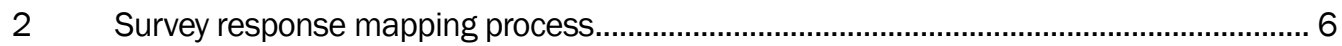

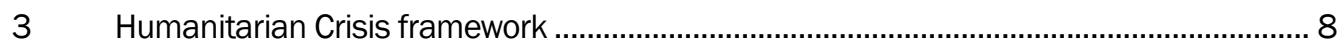

$4 \quad$ Heat map of twitter activity in the Philippines, 21 November 2017 ............................... 10

$5 \quad$ Example of the Interactive Map produced by the FICUS Twitter Tool .............................. 11

$6 \quad$ Positive tweet example, Manila, Oct 2017 ....................................................................... 19

$7 \quad$ Negative tweet example, Manila, Oct 2017 ................................................................. 19

8 The social media use framework ................................................................................ 21

9 Alignment of survey data to the social media use framework .........................................22

10 Normalizing survey responses. Each question response is normalized based on a range of values between 0 and 1 representing the possible extend of risk contribution ..............................................................................................2

11 Twitter sentiment map augmented by social media framework. The top left map shows sentiment-scored tweets; the bottom left shows the results of the social media use likelihood framework; and the right shows the results of processing both maps through map algebra .............................................................. 28

12 Manila infrastructure tweets-all, October 2017, ........................................................... 31

13 Manila infrastructure tweets limited to five per user, October 2017 …........................... 31

\section{Tables}

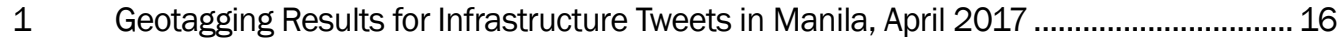

2 Geotagging results for infrastructure tweets in Manila, October 2017 .......................... 17

3 Geotagging results for infrastructure tweets in Metro Manila, October 2017

$4 \quad$ Raster calculations for augmenting tweet sentiment scores..........................................28 


\section{Preface}

This study was conducted for the Office of the Assistant Secretary of the Army for Acquisition, Logistics, and Technology under Research, Development, Test, and Evaluation (RDT\&E) Program Element 622784T41, "Military Facilities Engineering Technology"; Project P2 458304, "Framework for Integrating the Complexity of Urban Systems (FICUS)." The technical monitor was Ritchie L. Rodebaugh, Technical Director for Geospatial Research and Engineering.

The work was performed by the Land and Heritage Conservation Branch of the Installations Division, U.S. Army Engineer Research and Development Center, Construction Engineering Research Laboratory (ERDC-CERL). At the time of publication, Dr. Michael L. Hargrave was Chief, Land and Heritage Conservation Branch; Michelle J. Hanson was Chief, Installations Division; and Ritchie L. Rodebaugh was the Technical Director for Geospatial Research and Engineering. The Deputy Director of ERDC-CERL was Dr. Kirankumar Topudurti and the Director was Dr. Lance D. Hansen.

COL Teresa A. Schlosser was Commander of ERDC, and Dr. David W. Pittman was the Director. 


\section{Introduction}

\subsection{Background}

The Framework for Integrating the Complexity of Urban Systems (FICUS) project was sponsored by the Office of the Assistant Secretary of the Army for Acquisition, Logistics, and Technology (ASA(ALT)) for the purpose of empowering military planners with new tools for conflating and synthesizing data and knowledge that are critical for understanding megacities and other dense urban environments (DUEs). The purpose of the FICUS research effort was to design and develop a computational framework to support federated models of complex urban systems and facilitate improved levels of information support to Joint Intelligence Preparation of the Operational Environment (JIPOE) undertakings (Ehlschlaeger et al. 2018). Understanding DUEs requires integrated, accurate regional- and neighborhood-scale conceptual and computational models detailing operationally relevant information about the environmental, infrastructural, and social systems.

In developing the overall FICUS capabilities, a subset of ERDC researchers demonstrated how broad theory-based frameworks can inform more specific risk-evaluation frameworks and vice versa. The ability to link and fluctuate between theory and data-driven frameworks enhances the JIPOE process by representing the full range of complexity in urban systems.

One task in the overall development and validation of FICUS was to demonstrate how broad, theory-based frameworks can inform and be informed by more specific risk-evaluation frameworks. The ability to link and alternate between theory- and data-driven frameworks can enhance the JIPOE methodology by representing greater ranges of complexity in urban systems. In previous work, ERDC researchers completed a pilot application of FICUS to a theoretical framework for assessment of Humanitarian Crisis (HC) risk factors, which was provided by the United States Pacific Command (USPACOM) Joint Intelligence Operations Center (JICPAC) (Bastian et al. 2019). Next, the research team developed a cholera risk analytical framework that incorporated portions of that theoretical $\mathrm{HC}$ risk framework that are relevant to cholera outbreaks and epidemics. When the researchers applied FICUS methods to large, diverse social science datasets they were able to identify previously unavailable quantitative knowledge of significance in 
mitigating cholera risk and managing responses to outbreaks (Bastian et al. 2018).This data-driven demonstration also provided results that were used to improve the $\mathrm{HC}$ framework.

The present project again applied the FICUS data-conflation model and portions of the $\mathrm{HC}$ risk framework, which portrays risk in terms of five high-level conditions:

- Natural hazards

- Human behavior impact

- Services failure

- Readiness and response inadequacy

- Resilience deficiencies.

The topic of interest for the framework used in this FICUS case study is social media-Twitter in particular. This social media application had more than 300 million users worldwide as of mid-2017 (Wagner 2017). Twitter is similar to a texting application, but users can instantaneously share short messages globally to any of a virtually unlimited number of topical categories. These categories, known as hashtags, may represent a community of users interested in topics ranging from politics, social issues, or celebrity culture to pets and personal hobbies. A hashtag is delimited with an initial pound sign (\#) followed without a space by a subject term-for example, \#spambots or \#caturday. Within any hashtag messaging thread, participants may reply to any user message posted to Twitter, whether to validate someone's message, rebut it, or begin a longer-form conversation. Worldwide, Twitter is enthusiastically used by people from almost all socioeconomic strata, from young people in the developing world to the most powerful political leaders on the planet.

Twitter has been the topic of several studies related to humanitarian assistance and disaster relief (HADR) and epidemiology (Szomszor et al. 2010, Salathe et al. 2011, MacLean 2015, Meier 2012, Collins 2013, Kumar et al. 2011, Kumar et al. 2014, Cooper et al. 2015, Cassa et al. 2013, Dredze et al. 2013, Andrei et al. 2016). Some of this research has addressed topics reflected in other FICUS frameworks. More examples of Twitter research topics and analyses can be found in Steiger et al. (2015). The platform's open-source public API makes Twitter a readily available source of data for qualitative research that can augment the value of more quantitative research in near-real time. In the present FICUS case study, an open- 
source Twitter data collection and sentiment analysis model was developed and combined with a data-driven social media use computational framework in order to (1) better quantify the uncertainties inherent in social media data and (2) reduce uncertainties in the model.

\subsection{Objective}

The objective of this case study was to demonstrate how broad military operational frameworks can inform be informed by social media analysis. At this phase of study, the goal was to demonstrate and validate the quantitative use of FICUS with large-scale existing datasets to improve the quality of information provided to military decision makers.

\subsection{Approach}

Chapter 2 presents a review of the FICUS data-conflation methodology and a discussion of previous applications of FICUS to social science frameworks. It also explains the significance of social media usage data in the Philippines, which is the subject nation for this case study. Chapter 3 describes the development of an open-source FICUS Twitter data-conflation tool, and Chapter 4 describes development of the social media use data framework. Chapter 5 concludes the report with a discussion of the anticipated techniques and applications of the open-source FICUS Twitter tool and its complementary social media use framework. The Appendix shows the content of the Social Media User framework presented within the FICUS data architecture. 


\section{FICUS Demographic Models}

\subsection{Technology overview}

ERDC-CERL researchers developed a methodology and model for representing multifarious sociocultural data layers in a way that end users will understand. This technology, called FICUS (Ehlschlaeger et al. 2018), can provide end users more informative, data-based insights into complex situational issues that affect military planning and operational activities. The central capability of FICUS is a data-conflation model that can combine massive demographic databases into GIS map layers of social, infrastructural, and environmental metrics that can be aligned to mission-specific operational use. Unique to the FICUS model is its ability to account for all input data error and model uncertainties using statistically rigorous methods. The FICUS model requires that source data be constructed using a spatial-temporal uncertainty model presenting alternative representations of the data layers based on the known errors and uncertainties.

The model uses data from a subject nation's census, the U.S. Agency for International Development (USAID 2011), and DoD-sponsored surveys. It also requires subject matter experts (SMEs) to represent the range of framework-weighting factors based on their knowledge of the completeness of the available data sources for operational needs. Monte Carlo simulation is then applied to survey responses as they are applied to the framework, creating a range of likely results for each of level of framework components (i.e., conditions, factors, indicators).

The FICUS presentation of a range of prospective results allows decision makers to understand the utility of the available data. The results are generated in the form of geospatial thematic maps at a resolution of 200 meters per grid cell. Each grid cell contains the range and distribution of possible metric values for the population within 800 meters of that cell's location. Figure 1 shows a representative example of metric outputs from the FICUS model produced for previous studies. 
Figure 1. Estimated average wealth inequity between Muslims and Hindus near Dhaka, Bangladesh.

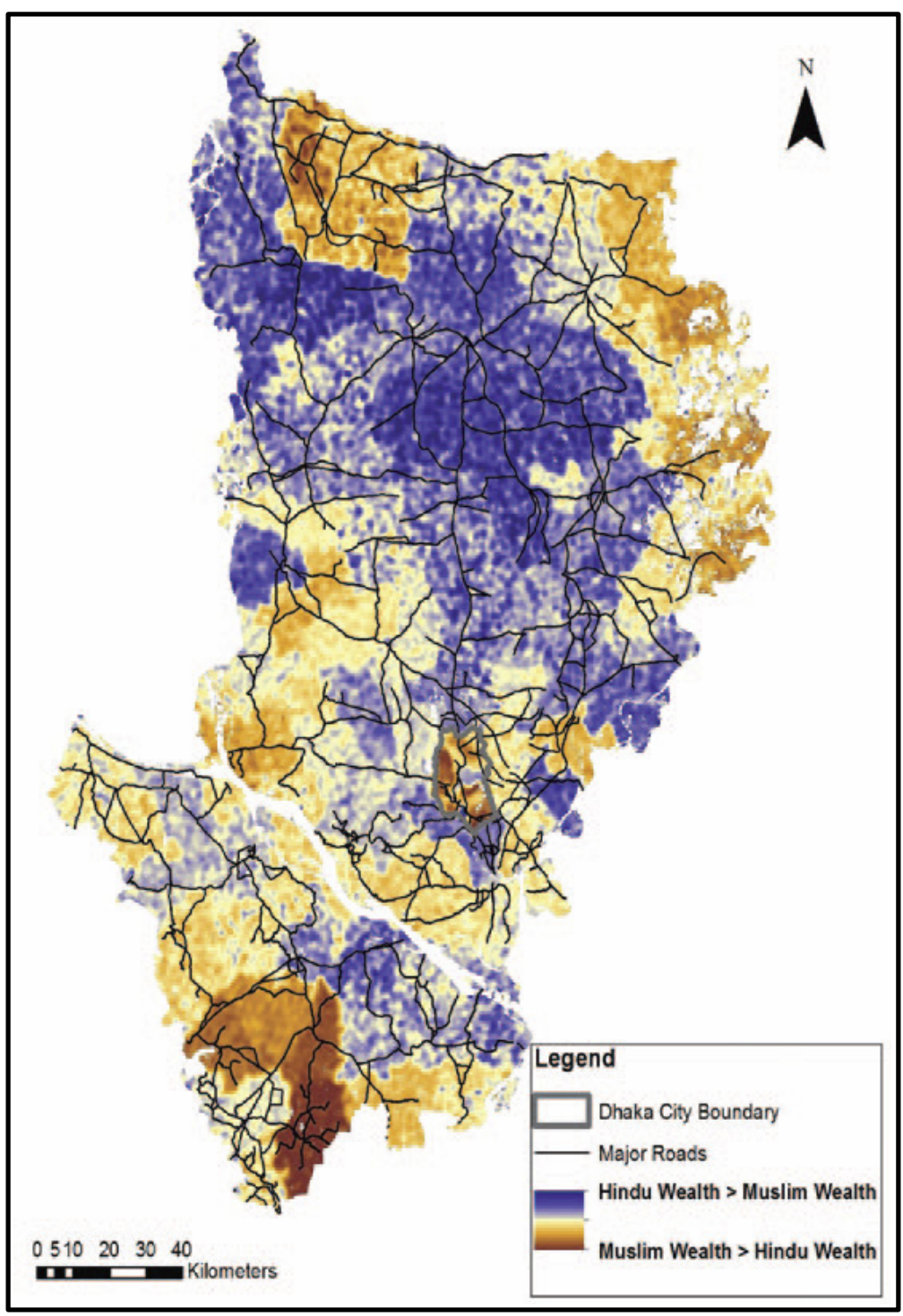

Details of the FICUS model are published in (Ehlschlaeger et al. 2016). Overall, the technique is composed of six steps, illustrated in Figure 2. The first three steps focus on simulating the population within the landscape. This phase includes accurately representing population densities and fitting population demographics within that representation. Key to this is understanding the environmental factors that influence the attractiveness of a site for a household to locate. The last three steps generate indicator maps of the simulated households. These steps focus on retaining the errors and uncertainties of the input data in a way that enables end users to understand the impacts on their application and ultimately improve the utility of the information for decision makers. 
Figure 2. Survey response mapping process.

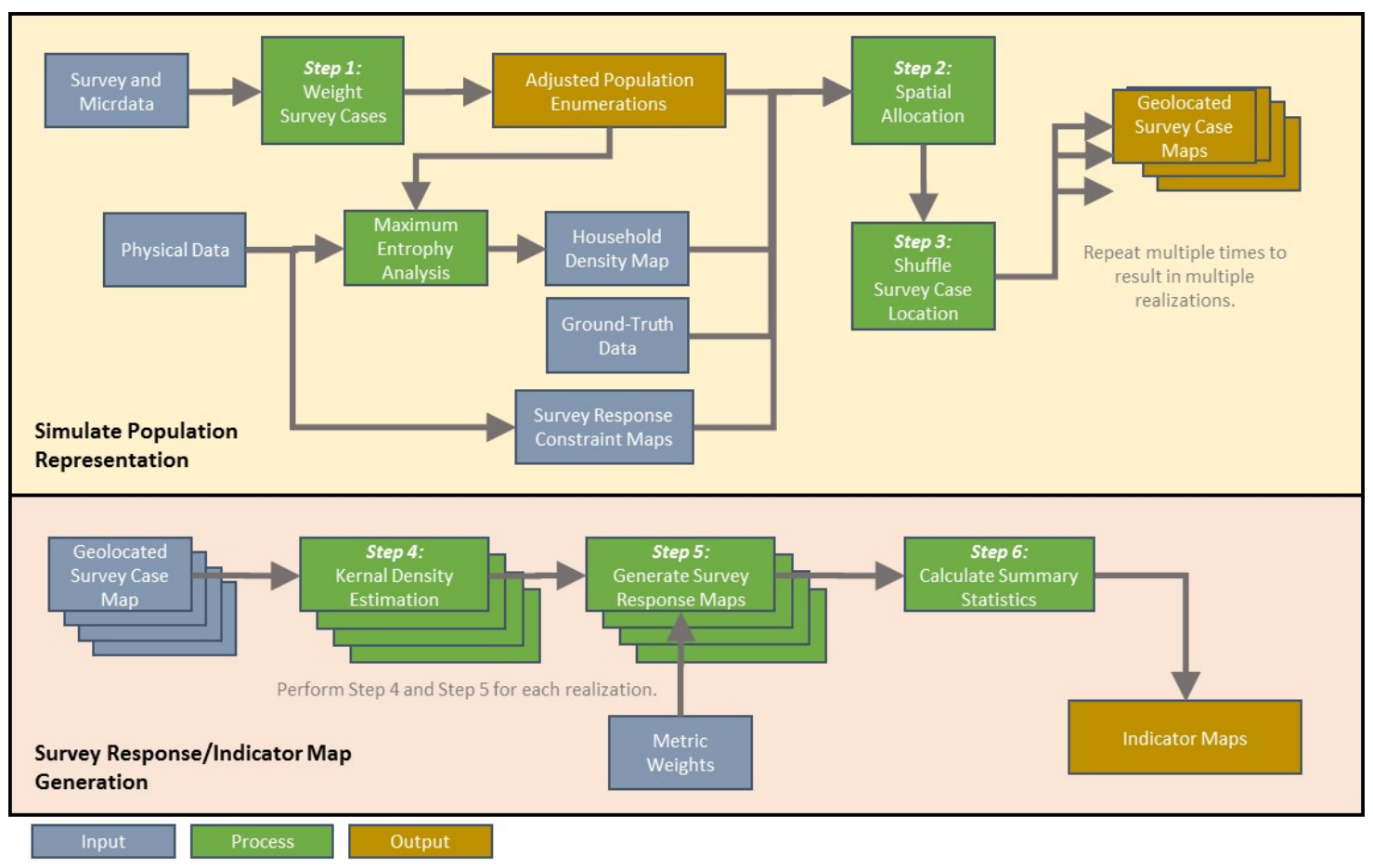

Each step of the FICUS methodology is briefly explained below:

1. Weight Survey Cases. Survey cases are replicated a number of times to match demographic characteristics in the overall estimated population enumerations. The replication process fits the results and are weighted using a sum of least squares minimizing specific desirable survey responses.

2. Spatial Allocation. Household survey cases are realized into plausible geographic locations. Ultimate household location maps are based on household density maps, ground truth data, and survey shuffling for optimization. The process of maximum entropy analysis generates household density maps.

3. Shuffle Survey Case Location. Survey case locations are shuffled to improve spatial statistics. This optimizes a set of proportional and spatial statistics for each population realization to create realistic clustering of survey responses.

4. Kernel Density Estimation. For each desired combination of survey responses, proportion maps are generated on each population realization throughout the study area, representing the percentages of simulated survey cases with such responses. This kernel density estimation process is done cell by cell across a regularized grid.

5. Generate Survey Response Maps. Map algebra analysis generates survey response maps $1-n$ realizations. 
6. Calculate Summary Statistics. Throughout the study area, box plot summary statistic maps are compiled on the minimum, maximum, median, medium, first quartile, and third quartile of realizations at all study area locations, as well as the standard deviation and interquartile range for these locations. Both the summary statistics and the kernel analysis for each realization provide error and uncertainty estimates.

7. Steps 1-4 are repeated for dozens, hundreds, or even thousands of alternative realizations in order to create enough realizations to provide representative distributions for important survey answers at critical geographic locations. For example, a survey response that is seldom answered by respondents would require a larger number of realizations for its Poisson distribution to reflect the variation of reality while a survey response answered by about 50\% of the households or people would take fewer realizations to define the resulting normal distribution.

\subsection{Initial humanitarian crisis framework}

Figure 3 depicts a theoretical HC framework of conditions, factors, and indicators developed for a previous FICUS study (Bastian et al. 2109). The present case study is informed by this prototype HC framework. Each framework category, from the higher to lower levels indicated by the framework column headings as read from right to left, measures a different dimension that represents a potential risk or stressor. JICPAC provided this prototype theoretical $\mathrm{HC}$ framework and chose Bangladesh as the subject of a case study to demonstrate the usefulness and functionality of a computational framework for better-informed decision making.

The purpose of the prototype $\mathrm{HC}$ framework is to show estimated risk levels in a way that allows for comparison within and across nations. The HC framework, and others developed using the same methods (including FICUS data conflation), encompass three categorical levels-conditions, factors and indicators. At the highest framework level shown in Figure 3, each condition encompasses a broad category of events and circumstances that cause or lead to humanitarian crises. The first HC framework condition, Natural Hazards, encompasses crisis events that often occur within a short temporal range but seriously affect large populations and produce ramifications for years to come. 
Figure 3. Humanitarian Crisis framework.

\begin{tabular}{|c|c|c|}
\hline Conditions & Factors & Indicators \\
\hline \multirow{11}{*}{$\begin{array}{l}\text { Natural } \\
\text { Hazards }\end{array}$} & \multirow{3}{*}{$\begin{array}{c}\text { Geophysical } \\
\text { Effects/Consequences }\end{array}$} & Earthquake/Earth Movement \\
\hline & & Landslide \\
\hline & & Volcanic Activity \\
\hline & \multirow{4}{*}{$\begin{array}{c}\text { Climatological } \\
\text { Effects/Consequences }\end{array}$} & Flood \\
\hline & & Severe Storm (thunderstorm, hurricane, tornado, cyclone, winter storms, etc.) \\
\hline & & Tsunami/Tidal Surge \\
\hline & & Drought \\
\hline & \multirow{4}{*}{ Ecosystem Disruptions } & Epidemics/Pandemic \\
\hline & & Severe Insect Infestation \\
\hline & & Commodity Destruction/Infection \\
\hline & & Wildfire \\
\hline \multirow{12}{*}{$\begin{array}{c}\text { Human- } \\
\text { Behavioral } \\
\text { Impact }\end{array}$} & \multirow{4}{*}{ Contamination and Degradation } & Food Processing Contamination \\
\hline & & Chemical or Other Industrial \\
\hline & & Nuclear/Radiological \\
\hline & & Biological (Animal Husbandry) \\
\hline & \multirow{4}{*}{ Vulnerable Groups } & IDPs/Refugees \\
\hline & & Undernourished Population (Not Gov't Priority, Resiliency) \\
\hline & & Casualties, Injured, Sick \\
\hline & & Targeted Groups (Genocide/Violence) \\
\hline & \multirow{4}{*}{ Substandard Conditions } & Civil Infrastructure Failure (Dams, Levies, Bridges, Buildings, Roads) \\
\hline & & Hazardous Working Conditions (Mine, Ag, Industrial) \\
\hline & & Overcrowding and Dangerous Housing \\
\hline & & Polluted and Disease-Bearing Environment (Swamps, Etc.) \\
\hline \multirow{12}{*}{$\begin{array}{c}\text { Services } \\
\text { Failure }\end{array}$} & \multirow{4}{*}{$\begin{array}{c}\text { Law Enforcement/Policing } \\
\text { Deficiencies }\end{array}$} & Policing/Patrol Deficits \\
\hline & & Inadequate Investigations and Prosecution \\
\hline & & Prisons and Jails (lack of capacity) \\
\hline & & Inadequate Facilities/ Property Protection \\
\hline & \multirow{4}{*}{$\begin{array}{l}\text { Health \& Medical Service } \\
\text { Insufficiencies }\end{array}$} & Doctor (Health Care Professionals) and Access to Primary Care \\
\hline & & Hospitals/Clinics and Secondary Care (Medical Specialists) \\
\hline & & Availability of Pharmaceuticals (Antibiotics) \\
\hline & & Delayed or Deficient Mortuary Affairs \\
\hline & \multirow{4}{*}{ Utilities Disruption } & Inadequate Sanitation \\
\hline & & Water Shortfalls \\
\hline & & Lack of Communications Availability \\
\hline & & Energy Deficits \\
\hline \multirow{12}{*}{$\begin{array}{l}\text { Readiness } \\
\text { and } \\
\text { Response } \\
\text { Inadequacy }\end{array}$} & \multirow{4}{*}{$\begin{array}{c}\text { Lack of Preparation and } \\
\text { Warning }\end{array}$} & Pre-Positioning Supplies \\
\hline & & Hazard Plans, Personnel Training, and Exercises \\
\hline & & Established Detection Sensors (Buoy's Etc.) \\
\hline & & Dissemination/ Alert Broadcast Deficits \\
\hline & \multirow{4}{*}{$\begin{array}{l}\text { Emergency Response } \\
\text { Shortfalls }\end{array}$} & Inadequate Fire and EMS Services \\
\hline & & Extraction and Debris Removal (Equipment) \\
\hline & & Inadequate Search and Rescue \\
\hline & & Insufficient Lift/Evacuation, Transportation Capacity \\
\hline & \multirow{4}{*}{ Civil/Military Authority Failures } & [Lack of] Established / Delegated Authority \\
\hline & & Insufficient Interoperable Communications \\
\hline & & Lack of Technical/ Functional Response Competency \\
\hline & & Breakdown of Situational Awareness (COP) \\
\hline \multirow{12}{*}{$\begin{array}{c}\text { Resilience } \\
\text { Deficiencies }\end{array}$} & \multirow{4}{*}{$\begin{array}{c}\text { Insufficient } \\
\text { Investment/Assistance }\end{array}$} & Insurance/ Reinsurance \\
\hline & & Insufficient Loans/ Debt Forbearance and Cancellation \\
\hline & & Foreign/Federal Relief/Grants/Social Funds (Aid) \\
\hline & & Decreased Foreign Investment Based on Assessed Risk \\
\hline & & Absenteeism and Work Stoppages \\
\hline & Malfunctioning Commerce / & Production Decreases \\
\hline & Services & Failure of Normal Trade to Return \\
\hline & & Failure to Re-Establish Markets (Retail, Groceries, Etc.) \\
\hline & & Insufficient Rebuilding of Shelter/Housing \\
\hline & Inadequate & Sustained Lack of Operational Utilities (the Grid) \\
\hline & Construction/Reconstruction & Delays to Re-establishment of Transportation Systems \\
\hline & & Diminished Agricultural Production \\
\hline
\end{tabular}


The second HC condition, Human Behavioral Impact, addresses negative human impacts on the natural environment, people, and infrastructure/built environment that can potentially increase risk of a crisis. Service Failure, the third condition, focuses on the failure of organized entities (mostly public, but private ones may be included) to provide basic services to citizens. These services include factors such as the rule of law, policing/justice, health and medical care, and public utilities. The last two conditions-Readiness and Response Inadequacies, and Resilience Deficienciesprimarily account for systemic factors related to the nation's ability to accommodate, respond, and recover from humanitarian crises and disasters.

\subsection{Social media use in the Philippines}

Filipinos are some of the world's most avid social media users, making the Philippines an excellent location for Twitter analysis. Currently, $58 \%$ of the population are monthly active social media users (Third Team Media 2017). Filipinos spend more time (4.17 hours per day) on social media than any other country in the world (Third Team Media 2017). While Facebook is the most popular social media platform in the Philippines with over 1.87 billion active users, Twitter is still in the top 10 with over 317 million active users (Third Team Media 2017). Filipino Twitter users have been shown to more actively engage with the platform versus other global users, with $69 \%$ of users engaging daily versus the global average of 33\% (Branding in Asia 2016). A heat map showing the percentage of the most recent million tweets in the Philippines shows that while tweet activity is clustered in the megacity of Manila, it is also widespread throughout the islands (Figure 4).

Given that social media has broadly penetrated the Philippines market, and that the use of social media has been proven to be effective in HADR crises, the research team sought to combine Twitter data with a computation framework specific to social media use. In this way, the uncertainty of tweet locations can be better calibrated against the framework model, and the result can potentially augment a broader military operational framework such as the HC framework. 
Figure 4. Heat map of twitter activity in the Philippines, 21 November 2017.

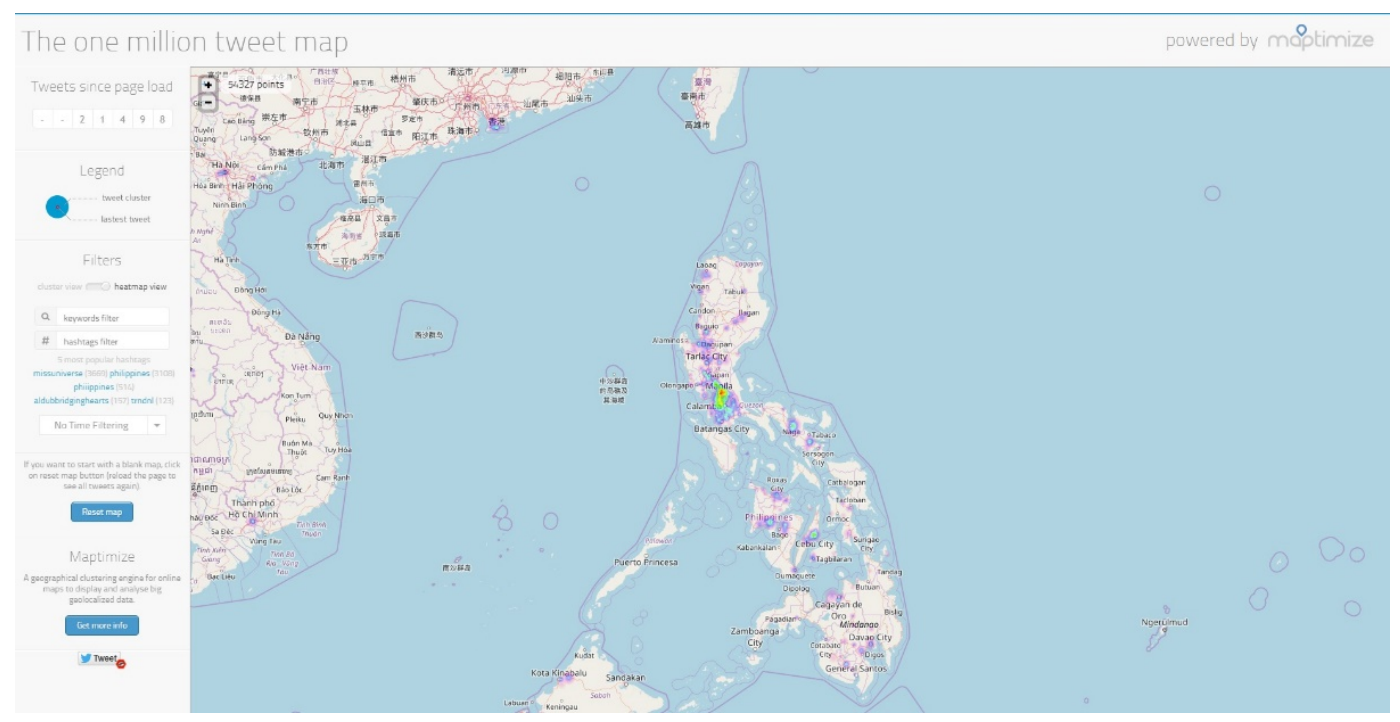

Source: https://www.onemilliontweetmap.com. 


\section{Developing the FICUS Twitter Tool}

The FICUS open-source Twitter tool consists of a series of R scripts integrated into the Open Modeling System (OMS) backend. These scripts can be accessed and run from any location with an internet connection and access to the cloud repository. The goal of the tool is for users to identify a region of interest, a "bag of words," and a time period (optional) with which to query Twitter, with the result being an interactive map printed in the browser. The map displays tweets color-coded by sentiment and averaged over a neighborhood grid cell (Figure 5).

For the first iteration of the FICUS Twitter tool, the region of interest was the City of Manila and the surrounding metro area. The "bag of words" was a list of 204 words compiled by the authors related to transportation and infrastructure in Manila, in both English and Filipino.

The tool can be broken down into four main components, depending on what kind of research and analysis a user is looking to complete. There four components are: Collection, Geotagging, Sentiment Analysis, and Visualization. Each component was built using the R language. They can be used as standalone tools or combined to run as a whole (the default for the FICUS Twitter tool).

Figure 5. Example of the Interactive Map produced by the FICUS Twitter Tool.

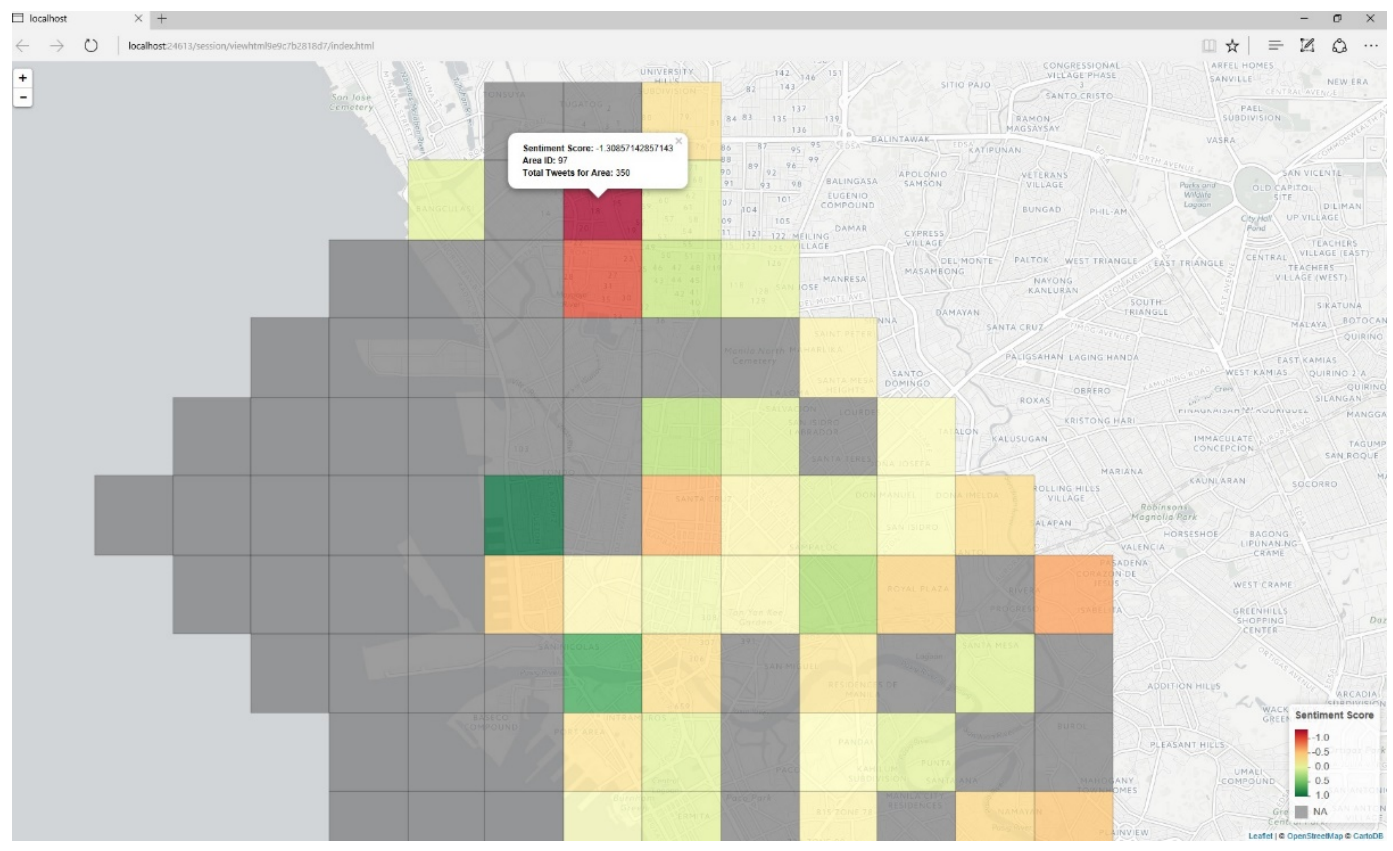


The Collection component allows users to collect and store tweets based on a defined geography, bag of words, and even a time period within the last 2 weeks. As the Collection component uses the Twitter Search API and not the Streaming API, it goes back in time from when the query is submitted to return results.

The Geotagging component attempts to match a percentage of tweets without geographic latitude and longitude coordinates to neighborhood areas (typically $1 \mathrm{~km} \times 1 \mathrm{~km}$ grid squares) based on the language of the tweets. The methodology and logic for this method is based on Paraskevopoulos et al. (2016).

The Sentiment Analysis component assigns a numeric sentiment score to each tweet, where a positive number denotes a positive sentiment and a negative number denotes a negative sentiment. Numbers are calculated based on the number of matches between dictionaries of positive and negative words (compiled by Liu et al. 2005 and translated into both English and Filipino) and the tweet text.

The Visualization component combines the visualization created by the grid cells color-coded to sentiment with other visualizations produced within the FICUS open-source OMS environment. The tweets can also be visualized on a desktop using both Leaflet and R/R Studio.

The following sections detail each component methodology and its logic.

\subsection{Collection}

The Collection component uses the public Twitter Standard Search API, which supports up to 7 days of tweet history from the point in time of the query (Twitter 2017). The public Search API allows free, rate-limited access to an average of $1-2 \%$ of the total tweets available; although limiting the query with a specific search term(s), geographic bounding box, etc., would theoretically increase the percentage of total tweets returned. Users can also access as sample of tweets in real time using the publicly available Twitter Streaming API. Other higher fidelity and reliable access to other Twitter streams are available for purchase from Twitter.

With an authorized connection to the Twitter public Search API, the Collection component will return the first 100 tweets per term in the "bag of 
words" for the pre-defined geographic boundary. The "bag of words" consists of a.csv file in which each word has its own row. The boundary is a circle identified by a center coordinate (latitude, longitude) and a radius distance. Given a shapefile (or other spatial dataset) of a city, state, or other geographic boundary, a GIS software (such as R or ESRI's ArcGIS) can be used to create a circular bounding geometry to determine the appropriate center coordinate and radius length. In the case of the FICUS Twitter tool prototype, the bag of words was a list of 203 words related to infrastructure in both English and Filipino (translated using Google Translate), ranging from the generic (i.e., "water," "transportation") to the specific names of major roads, transportation modes, and waterways in Manila (i.e., "Ninoy Aquino," "jeepney"). The bounding geometry was based on the City of Manila.

Tweet metadata that are downloaded for each tweet can include: tweet text, screenname, time and date stamp, tweet ID, favorite count, if the tweet is a retweet, if the tweet was a reply (and the screenname of the original tweet which is being replied to), tweet source, and latitude and longitude coordinates. The FICUS Twitter tool also adds a column with a time and date stamp for when the tweet was collected (i.e., the time of the query) and removes duplicate tweets based on the ID.

The Collection component stores all tweets in a table format within an SQLite database, and also exports them as a.csv file.

\subsection{Geotagging based on language modeling}

Geotagging tweets that are lacking geographic coordinates has been the subject of several scholarly studies (Chandra et al. 2011, Hironaka et al. 2016, Mahmud et al. 2012, Hecht et al. 2011, Wing et al. 2011, Schulz et al. 2013, Roller et al. 2012, Compton et al. 2015, Poulston et al. 2017). These methods range from using screennames of followers to in-text interactions between users ("mentions"), language analysis, profile information analysis, and external text corpi to identify the location from which the tweet was generated from and/or the home location of the user. Steiger et al. (2015), Table 2 provides a detailed review and study overview of papers conducting spatiotemporal Twitter analyzes (p. 14).

Despite the deluge of research that has been conducted in the past decade on geotagging tweets, no one methodology has proven to be better than the others. Obtaining the highest possible percentage of successfully geotagged 
tweets depends on the number of tweets in the dataset, the bounding geography, and desired granularity of geocoding (i.e., country, state, city, etc.). For HADR and other event-related efforts to use social media for crisis management, finer-grained geolocation techniques (i.e., below the city level) are not only more desirable statistically but can be vital to accomplishing operational mission goals. Ozdikis et al. (2017) published a review studies focused on of targeted event types and granularity of estimated locations, the majority of which are below the city level (Table 1, p. 299).

In general, research has shown that language (text) analysis of tweets, particularly when combined with other analysis geotagging methods, produces the highest percentage of accurately geotagged tweets at below a city-level scale (Flatow et al. 2015, Kinsella et al. 2011, Sadilek et al. 2012, Paraskevopoulos et al. 2016). As the methodology presented by Paraskevopoulos et al. (2016) was one of the more recently published methods for geolocalizing non-geotagged tweets, and was shown to be an effective geotagging approach for smaller spatial geographies and smaller datasets of tweets, it was chosen by the authors to be replicated and modified using open-source R code. This methodology analyzes the text of geotagged tweets clustered into grid cells based on the concordance and significance of each word, then compares these numbers to those of nongeotagged tweets to find a match. As such, SMEs knowledge of non-English language was not required.

\subsubsection{Geotagging methodology functions}

There are three main functions completed by this portion of the FICUS Twitter tool throughout the geotagging process. They include, in order of operation: assigning geotagged tweets from the raw tweet dataset to a specified neighborhood grid cell, calculating concordance and significance, and calculating similarity between neighborhood grid cells and non-geotagged tweets. All algorithms which perform these functions and were replicated in the FICUS Twitter tool R code can be found in Paraskevopoulos et al. (2016).

\subsubsection{Neighborhood grid cell assignment}

As in Paraskevopoulos et al. (2016), the FICUS team used $1 \mathrm{~km} \mathrm{x} 1 \mathrm{~km}$ grid cells as pseudo "neighborhoods." Larger grid cells or other neighborhood spatial boundaries (i.e., census sub-geographies, zip codes, etc.) could easily be substituted in place of the $1 \mathrm{~km}^{2}$ grid. 
To geolocalize non-geotagged tweets, geotagged tweets must be assigned to a neighborhood grid cell based on if their coordinates fall between the grid cell extents. The number of the grid cell becomes the geotagged tweets "Area ID." On average, approximately 1-2\% of all tweets in a raw dataset were geotagged and assigned an Area ID.

While it has proven incredibly difficult to find any resources published by Twitter which quantify the percentage of geocoded tweets received as a query result compared to the actual percentage of total geocoded tweets produced on the platform, work by Morstatter et al. (2013) found that the public API covered over $90 \%$ of the geocoded tweets available from the Twitter Firehose (a paid version of the API which allows access to nearly all tweets produced on the platform). Ergo, it is safe to assume that all geocoded tweets returned for a query on the public Twitter API are a robust representation of the geocoded tweets as a whole for that area.

\subsubsection{Concordance and significance}

In-text/language analysis, concordance is the number of appearances of each keyword (i.e., frequency), and significance is a weight based on the importance of each word in relation to other words in the text corpus. Concordance was calculated for both (1) the text corpus for all geotagged tweets in each grid cell, and (2) the text corpus of each non-geotagged tweet. Significance was calculated across all tweets, both geotagged and non-geotagged. By giving each keyword a weight (equal to concordance divided by total words multiplied by significance) (1) per grid cell and (2) per non-geotagged tweet, similarity can then be computed to geolocalize the non-geotagged tweets. Low weights (i.e., below 0.1) can also be filtered out to further refine results.

\subsubsection{Similarity computation}

To calculate the similarity, the magnitude (Euclidean norm) is extracted (1) for all grid cells and (2) for all non-geotagged tweets. The similarity computation function iterates through all non-geotagged tweets and performs the following steps:

1. For each non-geotagged tweet, find grid cell areas which contain the same terms within the tweet text.

2. If there is a match, multiply the weights of the keywords together. 
3. Sum all weight products for all keywords that appear in both the non-geotagged tweet and in the grid cell area tweets.

4. Divide this sum by the product of the grid cell magnitude and the non-geotagged tweets magnitude.

The result is a similarity number for each non-geotagged tweet for each potential grid cell location. A probability distribution is then created for each non-geotagged tweet by dividing the similarity result by the total sum of all similarities, which normalizes the results and allows for further uncertainty quantification and distribution. Currently, the FICUS Twitter tool selects the grid cell location candidate with the highest probability for each non-geotagged tweet as the tweet location.

\subsubsection{Model validity testing}

In order to test the validity and accuracy of the geotagging methodology used in the FICUS Twitter tool, a test code was written to iterate over all geotagged tweets in a raw Twitter dataset. Using Monte Carlo simulation and permutation, a percentage of tweets was randomly selected to act as "gold standard" geotagged tweets, while the rest acted as a sample dataset. The model was tested on two different tweet datasets related to infrastructure in the City of Manila: one from April 2017, and one from October 2017. The results can be seen in Tables 1 and 2, respectively. Table 3 contains the results from the same model run for the metropolitan Manila geography in October 2017

As the model iterated over the dataset 25 times at each set percentage for "gold standard" geotagged tweets, the tables display an average of the 25 runs per percentage. Distance error is calculated as a straight-line distance between grid cell centroids.

As the April 2017 dataset has the highest number of geotagged tweets per dataset (Table 2), this could be the cause behind the higher percentages of any results and correct results returned.

Table 1. Geotagging Results for Infrastructure Tweets in Manila, April 2017.

\begin{tabular}{|l|l|l|l|l|l|l|l|}
\hline \multirow{2}{*}{$\begin{array}{c}\text { Percent Geotagged } \\
\text { Tweets* }\end{array}$} & \multicolumn{3}{|c|}{ Any Result } & \multicolumn{2}{c|}{ Correct Result } & \multicolumn{3}{c|}{ Distance Error (km) } \\
\cline { 2 - 8 } & \multicolumn{1}{|c|}{ Count } & Percent & \multicolumn{1}{c|}{ Count } & Percent & \multicolumn{1}{c|}{ Min } & Mean & Max \\
\hline $1 \%$ & 4374 & $95.40 \%$ & 1844 & $40.23 \%$ & 1.002 & 4.841 & 12.381 \\
\hline $2 \%$ & 4330 & $95.45 \%$ & 1299 & $28.63 \%$ & 1.001 & 4.839 & 12.452 \\
\hline $5 \%$ & 4201 & $95.52 \%$ & 830 & $18.88 \%$ & 1.000 & 4.832 & 12.482 \\
\hline *Total tweets in dataset (n) $=4629$
\end{tabular}


Table 2. Geotagging results for infrastructure tweets in Manila, October 2017.

\begin{tabular}{|l|c|c|c|c|c|c|c|}
\hline \multirow{2}{*}{$\begin{array}{c}\text { Percent Geotagged } \\
\text { Tweets* }\end{array}$} & \multicolumn{2}{|c|}{ Any Result } & \multicolumn{2}{c|}{ Correct Result } & \multicolumn{3}{c|}{ Distance Error (km) } \\
\cline { 2 - 8 } & Count & Percent & Count & Percent & Min & Mean & Max \\
\hline $1 \%$ & 458 & $65.49 \%$ & 163 & $23.23 \%$ & 1.007 & 3.640 & 9.490 \\
\hline $2 \%$ & 454 & $65.60 \%$ & 151 & $21.85 \%$ & 1.004 & 3.711 & 9.870 \\
\hline $5 \%$ & 440 & $65.56 \%$ & 113 & $16.79 \%$ & 1.002 & 3.555 & 9.920 \\
\hline $10 \%$ & 418 & $65.65 \%$ & 89 & $13.97 \%$ & 1.002 & 3.704 & 9.965 \\
\hline$*$ Total tweets in dataset $(\mathrm{n})=707$ & & & & & & & \\
\hline
\end{tabular}

Table 3. Geotagging results for infrastructure tweets in Metro Manila, October 2017.

\begin{tabular}{|l|l|l|l|l|l|l|c|}
\hline \multirow{2}{*}{$\begin{array}{c}\text { Percent Geotagged } \\
\text { Tweets* }\end{array}$} & \multicolumn{2}{|c|}{ Any Result } & \multicolumn{2}{c|}{ Correct Result } & \multicolumn{3}{c|}{ Distance Error (km) } \\
\cline { 2 - 8 } & Count & Percent & Count & Percent & Min & Mean & Max \\
\hline $1 \%$ & 1573 & $70.29 \%$ & 413 & $18.46 \%$ & 1.077 & 6.176 & 25.086 \\
\hline $2 \%$ & 2558 & $70.33 \%$ & 378 & $17.05 \%$ & 1.076 & 6.116 & 25.598 \\
\hline $5 \%$ & 1510 & $70.31 \%$ & 290 & $13.49 \%$ & 1.076 & 6.254 & 27.385 \\
\hline $10 \%$ & 1430 & $70.28 \%$ & 250 & $12.28 \%$ & 1.076 & 6.235 & 28.468 \\
\hline$*$ Total tweets in dataset $(n)=2261$ \\
\hline
\end{tabular}

Despite the small number of tweets in the Manila October 2017 dataset (Table 3), the model returned the correct result (i.e., successfully matched the tweet to the correct grid cell) an average of $22 \%$ of the time, given the typical number of 1-2\% of geotagged tweets as a "gold standard" dataset.

Due to the larger geographic area for the tweets tested (listed in Table 3), the maximum distance error is much higher than those listed in Table 1 or 2. However, it should be noted that the mean distance error is only about 2-3 kilometers more than the tweets within the City of Manila proper. In the same vein, the percent of correctly geolocated tweets listed in Table 3 is only about $4 \%$ less than those listed in Table 2 . Thus, while accuracy reduces slightly when the spatial boundaries of analysis are expanded, it is not reduced to the point where the model is invalid.

Based on the results of validity testing of the FICUS Twitter tool geotagging model, the research team can confidently state that

- the geotagging model can assign approximately $65 \%-70 \%$ of non-geotagged tweets to a $1 \mathrm{~km}^{2}$ area grid cell within approximately $4.92 \mathrm{~km}$ of the actual location

- of the tweets assigned to a grid cell, approximately $20.15 \%$ of those tweets will reflect the actual tweet location 
- as $\mathrm{n}$ (the number of tweets in the dataset) increases, the percentages of both (1) tweets assigned to any area grid cell and (2) tweets assigned to the correct area grid cell, will increase.

\subsection{Sentiment analysis}

The Sentiment Analysis component calculates a numeric sentiment score for each tweet. A positive number correlates with a positive sentiment, and a negative number with a negative sentiment. Zero is neutral.

Using a positive and negative dictionary of words compiled by Liu et al. (2005) and translated into both English and Filipino (using Google Translate), this component iterates through each geotagged tweet searching for matches to both dictionaries in the tweet text. Each match to a positive word is given 1 point, while each match to a negative word is given -1 point. The final sentiment score is a sum of all points for the total tweet text. Figures 6 and 7, respectively, show examples of positively and negatively scored tweets from the Manila October 2017 dataset.

\subsection{Visualization}

The Visualization component takes the results of the previous three components and displays them in an interactive browser (Figure 2, p 6). The tweets can be displayed within the open-source OMS environment or visualized on a desktop using both Leaflet and R/R Studio.

Grid cells are color-coded according to the mean sentiment score of all geotagged tweets within the cell. These grid cells can be downloaded as a shapefile for further manipulation on a desktop. 
Figure 6. Positive tweet example, Manila, Oct 2017

\section{Bacon}

@yeheybacon

The new traffic scheme for jeepneys sa Sta. Lucia area HELPED A LOOOOOT with the traffic in Marcos Hway. LIKE A LOT

8:18 AM - 24 Oct 2017

5 Retweets $\mathbf{1 1}$ Likes

(2) 시요

Q $\backslash 7 \begin{array}{lllll}5 & 0 & 11 & \square\end{array}$

Figure 7. Negative tweet example, Manila, Oct 2017

Rose Anne DC

@gnnroseannedc

: CHER Bus transport (TYW 504) falls from Alabang skyway ramp due to loss of break.@MMDA says 30 patients suffered minor injuries

8:27 AM - 1 Oct 2017 from Manila City, National Capital Region

Q 2 七 $\quad$ O 


\section{Developing the Social Media Use Framework}

The HC framework was a theory or knowledge-driven framework; essentially, the framework components (conditions, factors, and indicators) were identified and developed based on established literature. Researchers then had to attempt to match the available data sources to the indicator requirements. For example, the indicator "Lack of Communications Availability" called for metrics reflecting the percentage of communication towers, DSL and fiber optic lines disabled by crisis; percentage of population with access to communication (radio, television, cell phones, internet, newspapers) (compared with historical data); and satisfaction with access to communication. Some of these data were accounted for within the national census and survey data (as detailed in section 2.1), but other data gaps remained. These data gaps are reflected in the higher uncertainty values for the indicator, which account for unknown components (section 3.4).

The social media use framework (Figure 8) was created in order to augment the $\mathrm{HC}$ framework with a more specific risk assessment and qualitative input data, and to demonstrate methods for reducing uncertainties and unknowns in computational frameworks. Researchers used a combined approach of knowledge-driven and data-driven methodologies to build the social media use framework. First, a literature review was conducted to identify indicators of social media use. Survey and census data sources were examined to see if they could inform new indicators (a data-driven approach). Individual data metrics were then grouped into indicators based on their similarity in addressing likelihood of social media use. In some cases, these new indicators were incorporated into $\mathrm{HC}$ framework factors. As researchers defined these indicators themselves, the uncertainty was automatically reduced.

Although it was informed by the HC framework in its inception, the social media use framework has the potential to inform the $\mathrm{HC}$ framework in turn. For example, the social media framework could be added as an additional metric within the "Delays to Re-establishment of Transportation Systems" indicator in the HC framework to better understand how opinions expressed on Twitter can complement infrastructure data to paint a broader picture of transportation efficiency following a crisis. Depending on the level of influence the user of the $\mathrm{HC}$ framework wants social media use/Twitter to have, the social media use framework could even be inserted at the factor or condition level. 
Figure 8. The social media use framework.

\begin{tabular}{|c|c|c|c|}
\hline \multirow{14}{*}{ 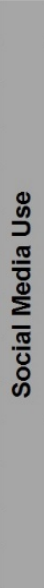 } & Conditions & Factors & Indicators \\
\hline & \multirow{6}{*}{ Population } & \multirow{3}{*}{ Individual Characteristics } & Sex \& Gender \\
\hline & & & Age \\
\hline & & & Disability \\
\hline & & \multirow{3}{*}{ Education } & Language Abilities \\
\hline & & & Literacy \\
\hline & & & Educational Attainment \\
\hline & \multirow{3}{*}{ Technology } & \multirow{2}{*}{ Device Access } & Phone Access \\
\hline & & & Computer Access \\
\hline & & Network Access & Internet Access \\
\hline & \multirow{4}{*}{ Place } & \multirow{2}{*}{ Household Characteristics } & Electricity \\
\hline & & & Wealth Index \\
\hline & & \multirow{2}{*}{ Urban Characteristics } & Road Network \\
\hline & & & Urban Proximity \\
\hline
\end{tabular}

\subsection{Input data}

The data currently informing the social media use assessment framework consist of national census microdata on the Philippines and the USAID Demographic and Health Survey (USAID DHS), as also mentioned in section 2.1. Figure 9 displays the conditions (teal), factors (purple), and indicators of the social media use framework, as well as the data informing each indicator. Indicators informed by only the USAID DHS results are in yellow. Indicators informed by both the USAID DHS and the census microdata are in bright green. Any indicators informed by external sources (blue) resulted in randomly generated maps that can be updated through the incorporation of non-survey data into FICUS.

Unlike the HC framework, nearly all of the data inputs for the social media use framework reflected quantitative, not qualitative, measures and metrics. For example, metrics for the indicator "Electricity" would measure if a household has access to electricity or not, rather than if they would consider access to electricity to be a big issue for their household.

\subsection{Risk value}

Each metric variable must be normalized for apples-to-apples comparisons. User assigns a range of values between 0 and 1 to all possible survey question responses representing the possible extent of risk contribution. Threshold values are shown in the Figure 10. A value of "o" equates to total chaos, while a value of " 1 " equates to no perceived risk. In the case of the social media use framework, a value of "o" equates to not at all likely to social media, while a value of "1" equates to near certain use of social media. 
Figure 9. Alignment of survey data to the social media use framework

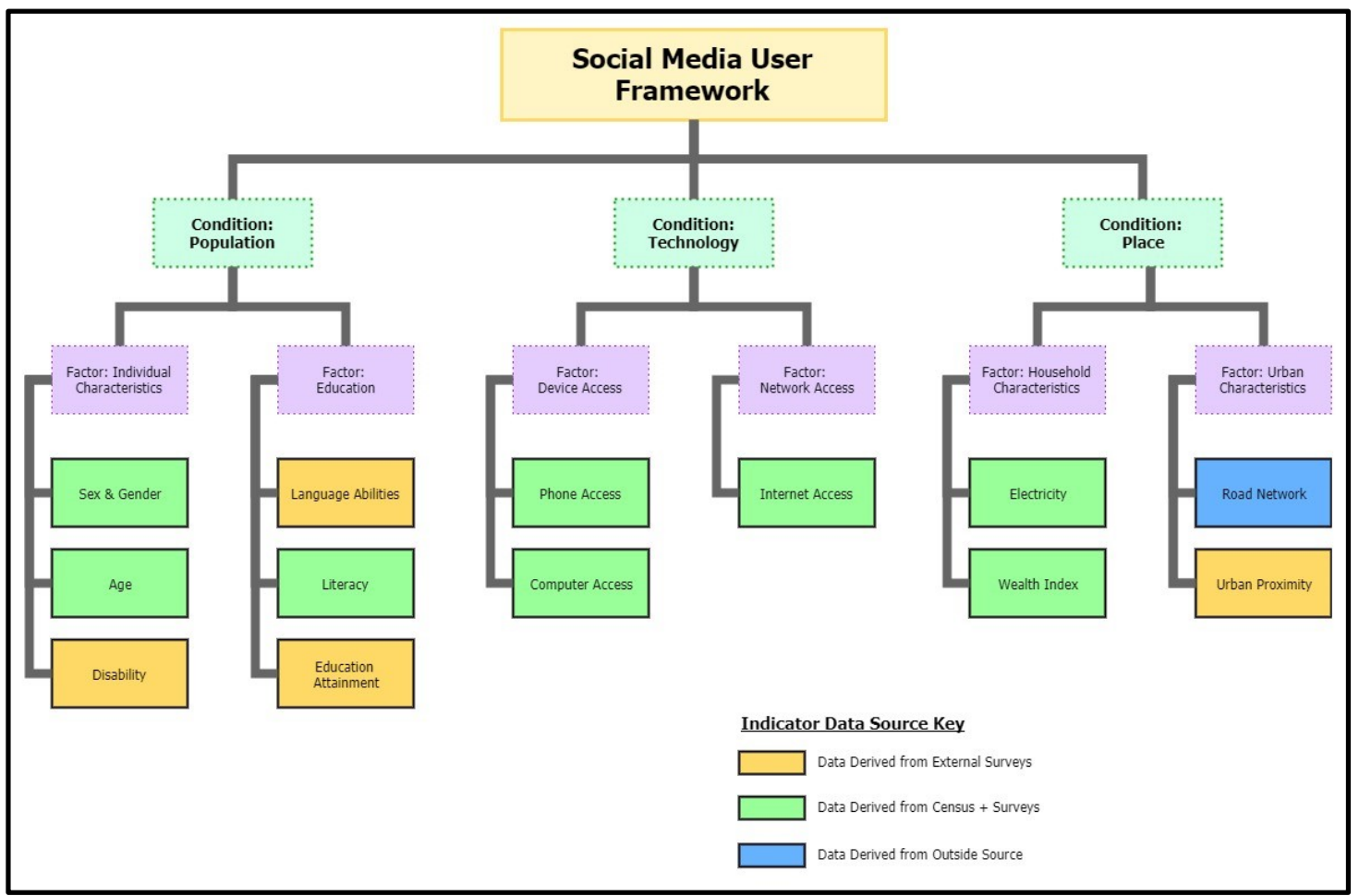

Figure 10. Normalizing survey responses. Each question response is normalized based on a range of values between 0 and 1 representing the possible extend of risk contribution.

\begin{tabular}{cll} 
Color & Metric Value & Description \\
\hline 1 & Minimal risk. Has no impact on risk. \\
\hline 0.75 & $\begin{array}{l}\text { Minimal risk. If the weights of all metrics with a .75 value sum to 2.0 or greater, } \\
\text { indicator will be slight risk or worse even if all other metrics have a 1.0 value. }\end{array}$ \\
\hline $500 \ldots 1$ & $\begin{array}{l}\text { Minimal risk. If the sum of all weights with a metric value of .500...1 is 1.0, any } \\
\text { other metric has a value < 1.0, indicator will be slight risk or worse. }\end{array}$ \\
\hline 0.5 & $\begin{array}{l}\text { Slight risk. If the weights of all metrics with a .5 value sum to 2.0 or greater, } \\
\text { indicator will be slight risk or worse even if all other metrics have a 1.0 value. }\end{array}$ \\
\hline $0.2500 \ldots 1$ & $\begin{array}{l}\text { Slight risk. If the sum of all weights with a metric value of .500...1 is 1.0, any other } \\
\text { metric has a value < 1.0, indicator will be medium risk or worse. }\end{array}$ \\
\hline 0.25 & Medium risk \\
\hline $.12500 . .1$ & Medium risk. \\
\hline 0.125 & High risk \\
\hline 0.0625 & Extreme risk \\
\hline
\end{tabular}

If the level of risk (or social media use) is uncertain, a range (min and max) of values may be selected. The wider the range, the less certain of the risk contribution. The tighter the range, the more certain of the risk contribution. 


\subsection{Weights}

Screening requires the evaluation of a combination of indicators. Multiple indicators are often aggregated into a factor or condition, usually for comparison across locations or to indicate change over time. Weights are assigned to metrics, indicators, factors, and conditions-allowing each component level to be rolled-up to the next. Components are weighted against the other components within that level (i.e., all the metrics in one indicator, all the indicators in one factor, etc.). Users define weights as a numerical value between $o$ and 1 based on contribution to risk, o being the least important/constraining on the next level up, and 1 being the most important/constraining. Again, weights can be defined in terms of a range given an uncertain risk contribution level.

If all weights within a grouping add up to 1 , then each unit contributes to the accumulation of risk. If anyone (or more) weights within a grouping equal 1, then it (or they) drive the overall risk.

\subsection{Unknown components}

We know that this is not a complete list of metrics, indicator, factors, or conditions. There are potentially other variables that were not considered. In this situation, users can opt to add an unknown component. This inserts a random value that accounts for additional variables. It is given a weight and treated the same as any other component.

\subsection{Roll-up computation}

\subsubsection{Favorability functions}

Favorability functions calculate the overall component values using the risk value and the weight. Favorability functions were originally known as sieve mapping (McHarg 1969) and also called map overlays. Before map overlay existed as a computer algorithm, clear acetate maps were inked at locations least favorable to an activity or land use. Stacking the acetate maps on top of each would provide a visual method to assessment each location's suitability or risk. Bonham-Carter (1995) described digital map overlay as favorability functions, which includes easier weighting of individual maps and exact measures of suitability or risk. Traditionally, there has been two general types of favorability functions used, additive based favorability (equation 1) known as weighted linear combination (WLC) (Malcwewski 2000), or constraint-based favorability function (CBFF) (equation 2). 


$$
\begin{aligned}
& \mathbf{M}_{\boldsymbol{y}}=\sum_{i=1}^{n} w_{i} \mathbf{M}_{i} / w_{i} \\
& \mathbf{M}_{\boldsymbol{y}}=\prod_{i=1}^{n} w_{i} \mathbf{M}_{i} / w_{i}
\end{aligned}
$$

where

$\mathbf{M}$ is a map with values between 0.0 and 1.0,

$\mathbf{M}_{y}$ is the resulting indicator or risk assessment map,

$\mathbf{M}_{i}$ is the $i^{\text {th }}$ of $n$ criteria maps, and

$w_{i}$ is the $i^{\text {th }}$ weight to its criteria map.

WLC based risk assessments have the problem when a large risk becomes masked by other no/low risks at the same location. For example, a location where gang violence creates a high risk should have high risk even though other criteria, such as well funded after school programs, indicates a low risk. On the other hand, CBFF risk assessment does not allow for groups of criteria to positively reinforce each other-every criterion constrains the indicator to the value of criteria and no higher.

An ideal favorability function will allow both additive and constraint-based characteristics to be declared at both within criteria map locations and the criteria map weighting. Equation 3, the Power-Based Favorability Function (PBFF), achieves this goal:

$$
\mathbf{M}_{r}=\prod_{i=1}^{n} \mathbf{M}_{i, r}^{w_{i}}, 1.0 \leq \sum_{i=1}^{n} w_{i}
$$

where

$\mathbf{M}$ is a map with values between 0.0 and 1.0, $\mathbf{M}_{r}$ is the $r^{\text {th }}$ realization map of indicator values, $\mathbf{M}_{i}$ is the $i^{\text {th }}$ of $n$ criteria maps, and $w_{i}$ is the $i^{\text {th }}$ weight to its criteria map.

While PBFF's equation (3) is less intuitive than WLC and CBFF, having criteria variables be the constant of a power function allows it to be equivalent to WLC when the criteria weights sum to 1.o. Also, PBFF allows criteria weights to act influence the indicator like both WLC and CBFF when criteria weights sum to be greater than 1.o. For example, if a criterion has a weight of 1.0, its indicator will have the same or lower risk value, just as 
in CBFF. Criteria with weight less than 1.0 in PBFF will allow the indicator to have a higher value when other criteria are positive. Thanks to the product function in PBFF, criteria map locations can also be constraints when those locations are given a value of 0.0 or other extremely low values.

Another benefit to using PBFF, similar to CBFF's, is in the calibration process. Risk model developers can adjust individual criteria map weights or the values within the criteria map locations to calibrate to the desired indicator values without having to adjust other criteria map weights. Traditional risk assessment techniques require carefully choosing the indicator's criteria weights, adjusting all of them to whenever calibration is done. PBFF provides for an opportunity for non-linear optimization algorithms, such as neural nets or genetic algorithms, to create criteria map weights as well as the function variables that minimize the errors to known indicator values.

\subsubsection{Quantifying errors and uncertainty}

A stated goal of the FICUS effort was to explicitly represent errors and uncertainties within all products. For the PBFF to specifically quantify uncertainty, equation 4 becomes the Uncertainty Quantified (UQ) PBFF.

$$
\mathbf{M}_{r}=\mathbf{M}_{u, r}^{w_{u}} \times \prod_{i=1}^{n} \mathbf{M}_{i, r}^{w_{i}}, 1.0 \leq w_{u}+\sum_{i=1}^{n} w_{i}
$$

where

$$
\begin{aligned}
& \mathbf{M}_{u, r} \text { is the } r^{\text {th }} \text { realization map of simulated uncertainty for an } \\
& \text { indicator, and } \\
& w_{u} \text { is the weight of that uncertainty. }
\end{aligned}
$$

The simulated uncertainty map should be a random field of values between 0.0 and 1.0 with a histogram like the distribution of values within the criteria maps. The random field should have spatial autocorrelation to the largest spatial dependence of the criteria maps. For example, if the criteria maps used kernel analysis on demographic factors, the random field should have positive spatial autocorrelation equal to the kernel analysis diameter. While this research used the random field described in Ehlschlaeger (2002), there are many theoretical random field models to choose from, for example GSLIB (Deutsch \& Journel 1992). And while equation 4 explicitly represents the known uncertainties in the modeling process, the modelers were expected to represent the unknown uncertainties as well. With regard to UQ PBFF, modelers were expected to estimate the range of 
values for all weights, $w_{u}$ and $w_{i}$, that might exist accounting for the lack of perfect understanding between the criteria and the indicator. We asked the modelers to imagine which criteria they wish existed that would better explain the indicator. Then, modelers were to estimate which of those unavailable criteria had the least correlation with available criteria. Uncorrelated unavailable criteria would be indicated by higher values and greater ranges of the uncertainty weight $w u$. This uncertainty weight has the same behavior on the risk assessment model as the criteria weights. 


\section{Augmenting Twitter Sentiment Maps with Social Media Framework Maps}

This section describes how the results of the FICUS Twitter Tool (Section 2) and the Social Media Use Framework (Section 3) can be used in tandem. Uncertainty is inherent in both the geotagged, sentiment-coded tweet results and in the social media use framework results. However, through the combination of these results using raster calculation and map algebra, overall uncertainty can be reduced, and more informed datadriven decisions can be made within the operational environment.

The results of the social media use framework quantify, on a $200 \mathrm{~m} \times 200$ $\mathrm{m}$ neighborhood level, the likelihood of people living in that grid cell using Twitter and other forms of social media. By overlapping the tweet sentiment grid with the framework results, areas with strong sentiments (either positive or negative) can potentially be amplified or tempered based on whether or not there is a high likelihood of social media users within that grid cell. In this way, a methodology is provided that can help prevent certain tweet/Twitter users displaying strong sentiment from skewing the grid map (Figure 11).

The augmentation process was completed in three steps. First, the sentiment grid map was projected and rasterized with $200 \mathrm{~m} \times 200 \mathrm{~m}$ grid cell size (the same as the framework map results). Second, a new raster was created by reclassifying the framework raster based on the value of the framework map (Table 4). Using power (square) numbers (as is done with the framework components and metric metadata weighting scale), cells with values closer to 1 are given a higher number in order to better amplify sentiment scores in areas with a greater likelihood of frequent social media usage. Third, a raster calculator is used to multiply the reclassified framework raster and the tweet sentiment raster to produce an augmented sentiment raster map (Figure 8).

As seen in Figure 8, the augmented map filters out several of the stronger sentiment grid cells, with two adjacent cells showing the strongest negative and positive sentiments. Logically, the area correlating with these grid cells would be the first area of interest (AOI) for warfighters in the operational environment. The augmented maps allow for more informed course of action planning, and for making better data-driven decisions with less uncertainty. 
Figure 11. Twitter sentiment map augmented by social media framework. The top left map shows sentiment-scored tweets; the bottom left shows the results of the social media use likelihood framework; and the right shows the results of processing both maps through map algebra.

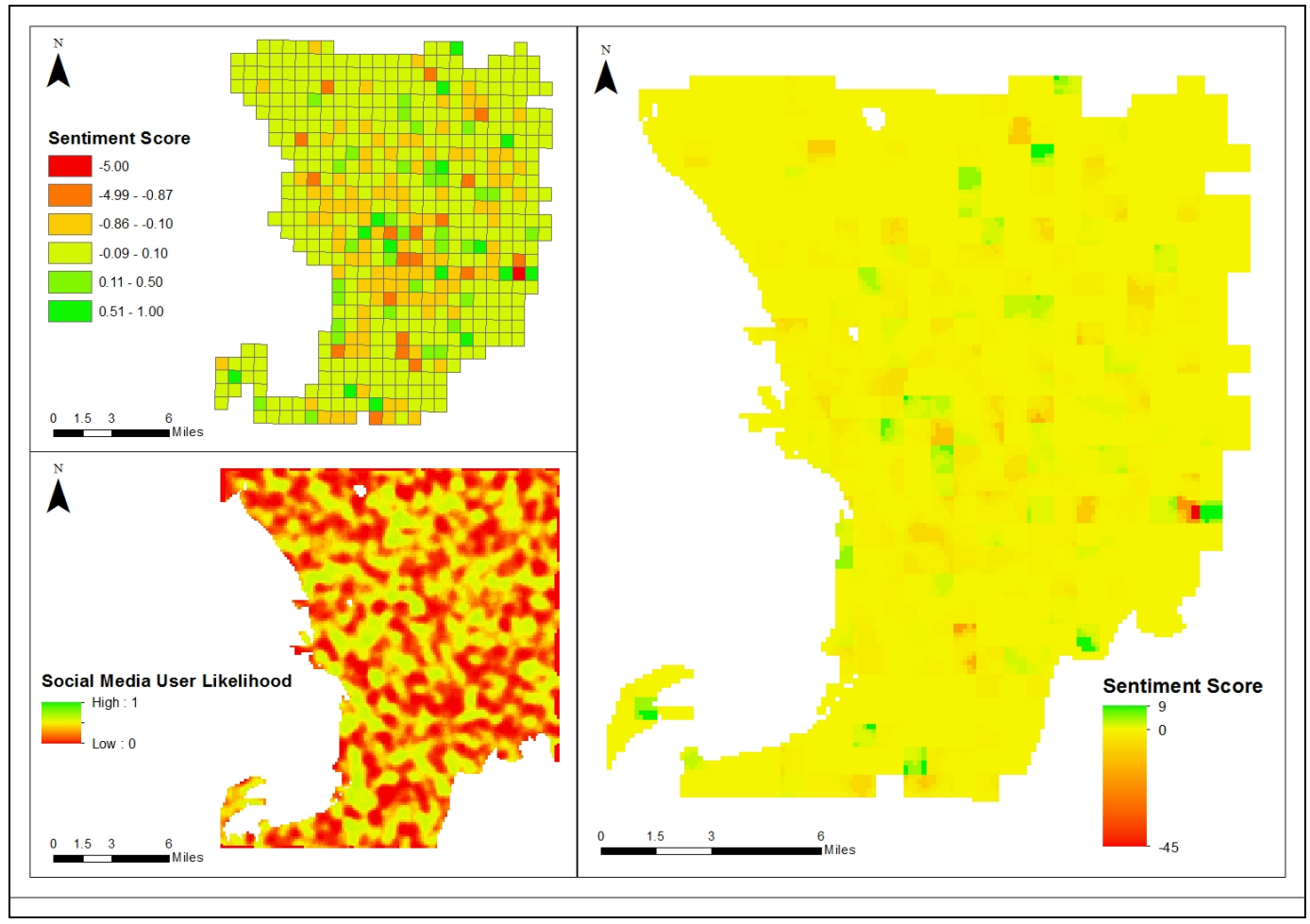

Table 4. Raster calculations for augmenting tweet sentiment scores.

\begin{tabular}{|c|c|}
\hline Framework Raster Values Range & New Augmentation Raster Values \\
\hline $0-0.0625$ & 1 \\
\hline $0.0625-0.125$ & 4 \\
\hline $0.125-0.25$ & 9 \\
\hline $0.25-0.5$ & 16 \\
\hline $0.5-0.75$ & 25 \\
\hline $0.75-1.0$ & 36 \\
\hline
\end{tabular}




\section{Conclusion}

The social media use framework, augmented with the FICUS Twitter tool, was created with the goal of demonstrating the ease of using a more general, knowledge-driven risk assessment framework (in this case, one which focuses on humanitarian crises) to inform a more specific, data-driven framework. Both the HC and social media use frameworks augment each other; and when used together, they can reduce overall uncertainties and increase overall awareness within the operational environment. Uncertainties are inherent, but rarely acknowledged, in both social media modeling and computational frameworks. This paper seeks to address both.

Implementing quantitative measures for the frameworks enables the ability to more easily compare changes as new data are made available. Further, it offers the ability to trace backward from the factors down to the metrics, so one can explore the impact of changing the weights of different components at the higher level, allowing accurate calibration of the analytic framework. Finally, the explicit accounting for uncertainty at each level allows analysts to more faithfully represent their understanding of the framework values, particularly when SMEs are not available. This reduces the uncertainty of those judgments.

There are likely to be gaps in the framework that are either not obvious or obscured by other framework components. Finding these gaps is vital to ensure the highest accuracy possible. This is a point where the hybrid approach, the incorporation of existing data and theoretical methods, can identify and address critical gaps in the framework. Both data availability and theoretical methods inform framework development in distinct ways. The geospatial risk maps provide intuitive methods for calibration and validation via qualitative techniques. When framework map errors are identified, there is an explicit connection to all modeling decisions and data streams to determine whether there is a logical flaw in the framework model or calibration is necessary to improve the analytic framework.

There are inherent issues with social media (and other big data) modeling that often go undiscussed (Ruths and Pfeffer 2014, Shelton 2017). According to Ruths and Pfeffer (2014), these include: the failure of APIs to accurately represent an entire platform's data (and the methodology behind choosing what results are returned being hidden); platform-specific behavioral norms; platform user bias; no canonical datasets available to compare 
(in a statistically sound manner) methodologies; proxy population mismatch, etc. Attempting to geolocate tweets without any spatial data also falls under these failures. It is worth noting again that there is no research-based best practice for geotagging and analyzing Twitter data. Larger datasets, larger geographies, and other factors could prove less successful with the methodology outlined here, and more successful with other methodologies from other research studies. However, by combining a geotagging via language modeling analysis with a computational framework, some of these failures and uncertainties are addressed and explicitly quantified.

In addition, there are other methods for reducing uncertainty overall. For example, instead of using grid cells of $1 \mathrm{~km} \mathrm{x} 1 \mathrm{~km}$, users of the FICUS Twitter tool could create neighborhood clusters similar to Flatow et al. (2015). One could also filter out time series/dates to allow for more eventrelated geolocation and Twitter analysis. Employing a SME who is familiar with the language and dialects of the AOI, as well as the geography, could greatly increase accuracy, both in creating the "bag of words" and in providing sentiment analysis. With their help, a training component could be added to the model. Accuracy can even be increased within the visualization component, as seen in Figures 12 and 13. Shelton et al. (2015)'s recommendation to limit tweets in a dataset to five per user slightly alters the mean sentiment scores of the grid cells, filtering out the possibility of overactive Twitter users to manipulate the dataset one way or the other.

The HC and Social Media Use framework exercise, along with the FICUS Twitter tool, serves as a good starting point for linking indicators to higher level planning objectives. With the addition of spatially representing quantitative metrics, incorporating uncertainty, and weighting the importance of individual components, analysts have the ability to more accurately and precisely communicate knowledge of the operating environment. This, in turn, provides a genuine pathway for the data-to-decisions paradigm. 
Figure 12. Manila infrastructure tweets-all, October 2017,

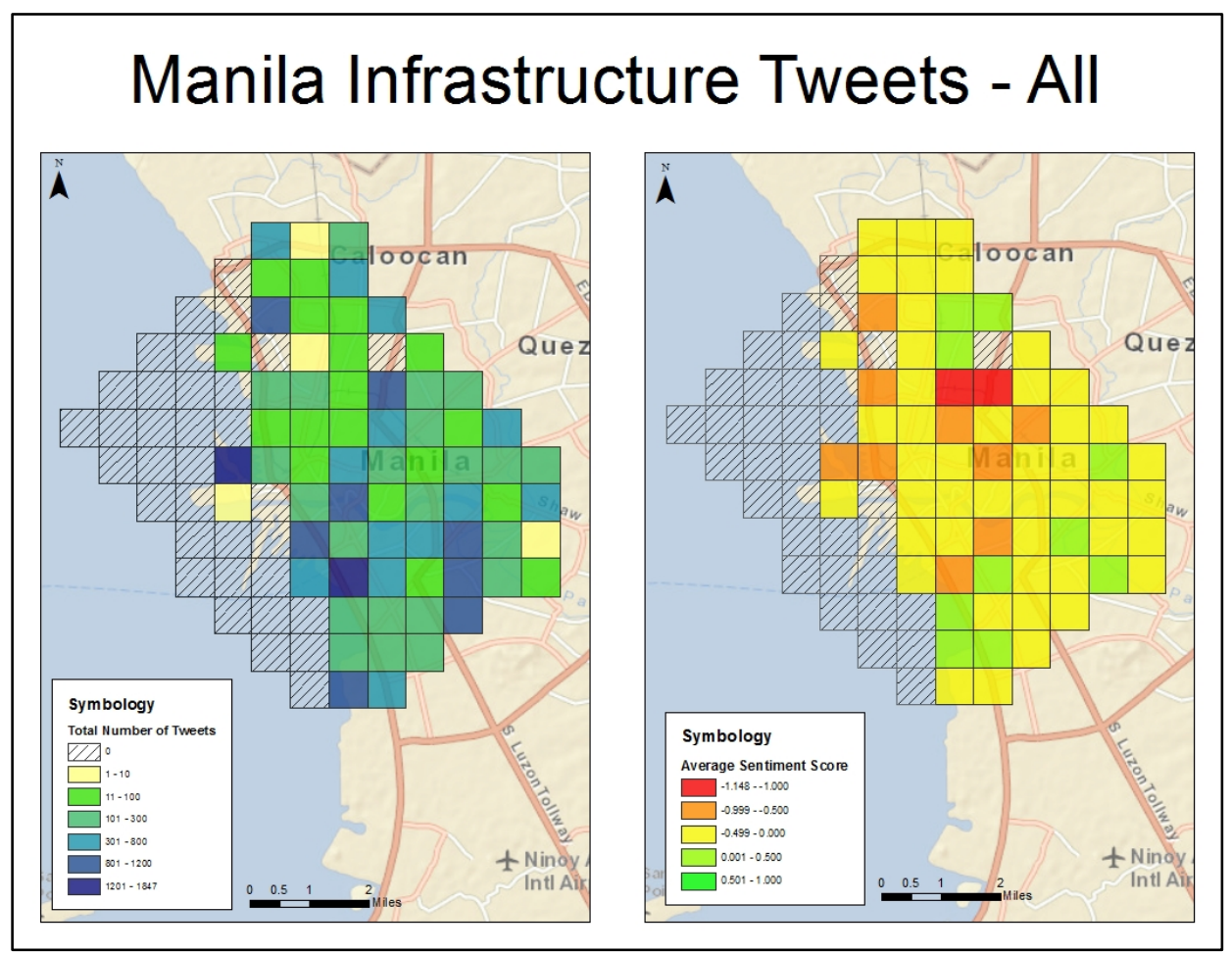

Figure 13. Manila infrastructure tweets limited to five per user, October 2017.

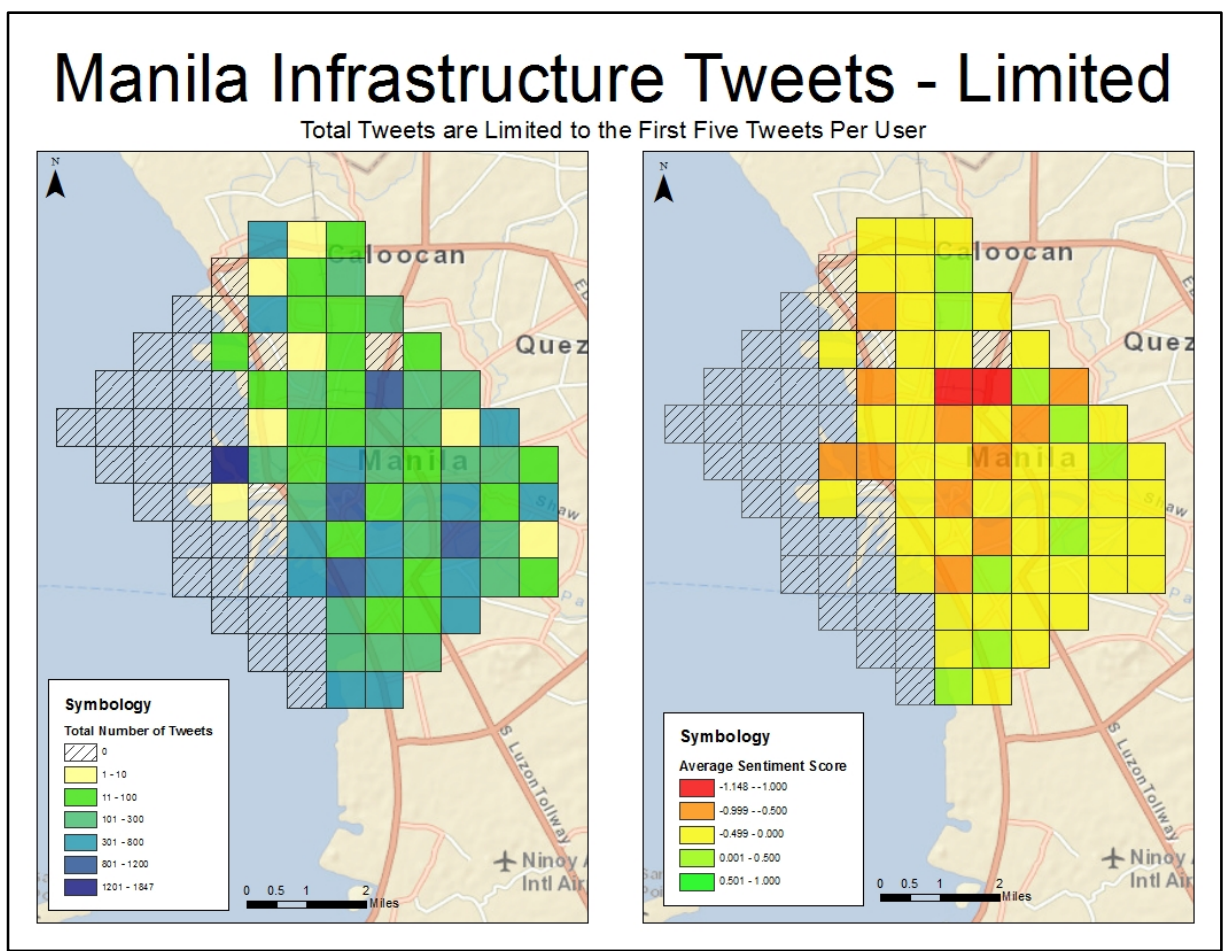




\section{Bibliography}

Albert, Jose Ramon G., Ramonette B. Serafica, and Beverly T. Lumbera. 2016. Examining Trends in ICT Statistics: How Does the Philippines Fare in ICT? Philippines Institute for Development Studies. https://dirp4.pids.gov.ph/websitecms/CDN/PUBLICATIONS/pidsdps1616.pdf.

Andrei, Amanda, Sara Beth Elson, and Guido Zarrella. 2016. Language and Emotion in Philippine Twitter Use during Typhoon Haiyan. ResearchGate. DOI 10.13140/RG.2.1.4671.5923.

Bastian, Elizabeth, Claire Munaretto, Natalie R. Myers, Carey L. Baxter, Jamie Fishman, James D. Westervelt, Charles R. Ehlschlaeger, and Jeffrey A. Burkhalter. 2019. Application of the FICUS Data-Conflation Model to a Theoretical Humanitarian Crisis Analytical Framework. ERDC/CERL TR-19-1. Champaign, IL: Engineer Research and Development Center, Construction Engineering Research Laboratory (ERDC-CERL). https://erdclibrary.erdc.dren.mil/xmlui/bitstream/handle/11681/32006/ERDC-CERL\%20TR-191.pdf? sequence $=1$ \&isAllowed $=y$.

Elizabeth Bastian, Claire Munaretto, Natalie R. Myers, Carey L. Baxter, Jamie Fishman, James D. Westervelt, Charles R. Ehlschlaeger, and Jeffrey A. Burkhalter. 2019. Development of a Cholera Epidemiological Risk Assessment Framework. ERDC/CERL TR-19-13. Champaign, IL: ERDC-CERL.

Branding in Asia. 2016. Twitter's Love Affair with the Philippines. 22 Feb 2016. https://brandinginasia.com/twitter-philippines/ on 21 Nov 2017.

Cassa, Christopher A, Rumi Chunara, Kenneth Mandl, and John S Brownstein. 2013. "Twitter as a Sentinel in Emergency Situations: Lessons from the Boston Marathon Explosions." PLoS: Current Disasters. http://currents.plos.org/disasters/article/twitter-as-a-sentinel-in-emergency-situations-lessonsfrom-the-boston-marathon-explosions/.

Chandra, Swarup, Latifur Khan, Fahad Bin Muhaya. 2011. "Estimating Twitter User Location Using Social Interactions - A Content Based Approach.” 2011 IEEE International Conference on Privacy, Security, Risk, and Trust, and IEEE International Conference on Social Computing. https://www.utdallas.edu/ lkhan/papers/Estimating\%20Twitter\%20User\%20Location\%20Usin g\%20Social\%20Interactions.pdf.

Collins, Katie, 2013. "How AI, Twitter and Digital Volunteers Are Transforming Humanitarian Disaster Response." Wired. https://www.wired.co.uk/article/digitalhumanitarianism.

Compton, Ryan, David Jurgens, and David Allen. 2015. "Geotagging One Hundred Million Twitter Accounts with Total Variation Minimization." Proceedings of the 2014 IEEE International Conference. 
Cooper, Guy Paul Jr., Violet Yeager, Frederick M. Burkle Jr., and Italo Subbarao. 2015. "Twitter as a Potential Disaster Risk Reduction Tool. Part I: Introduction, Terminology, Research and Operational Applications." PLoS: Current Disasters. http://currents.plos.org/disasters/article/twitter-as-a-potential-disaster-risk-reduction-toolpart-i-introduction-terminology-research-and-operational-applications/.

DHS (Demographic \& Health Survey). 2016. Bangladesh - Stat Compiler. http://dhsprogram.com/Where-We-Work/Country-Main.cfm?ctry_id=1\&c=Bangladesh.

Dredze, Mark, Michael J. Paul, Shane Bergsma, and Hieu Tran. 2013. "Carmen: A Twitter Geolocation System with Applications to Public Health.” Expanding the Boundaries of Health Informatics Using Artificial Intelligence: Papers from the AAAI 2013 Workshop. https://www.aaai.org/ocs/index.php/WS/AAAIW13/paper/download/7085/6497 on 21 Nov 2017.

Flatow, David, Mor Naaman, Ke Eddie Xie, Yana Volkovich, and Yaron Kanza. 2015. “On the Accuracy of Hyper-local Geotagging of Social Media Content." WSDM ' 15. https://arxiv.org/abs/1409.1461.

Greenwood, Shannon, Andrew Perrin, and Maeve Duggan. Social Media Update 2016. Pew Research Center. http://www.pewinternet.org/2016/11/11/social-media-update-2016/.

Hecht, Brent, Lichan Hong, Bongwon Suh, and Ed H. Chi. 2011. "Tweets from Justin Bieber's Heart: The Dynamics of the 'Location' Field in User Profiles.” CHI 2011. http://misrc.umn.edu/Papers/Research\%20Papers/bhecht_chi2011_location.pdf.

Hironaka, Shiori, Mitsuo Yoshida, and Kyoji Umemura. 2016. "Analysis of Home Location Estimation with Iteration on Twitter Following Relationship." 2016 IEEE. https://arxiv.org/pdf/1608.08331.pdf.

Kinsella, Sheila, Vanessa Murdock, and Neil O'Hare. 2011. “I'm Eating a Sandwich in Glasgow': Modeling Locations with Tweets." Proceedings of the 3rd International Workshop on Search and Mining User-Generated Content.

Kroulek, Alison. 2017. "Which Counties Have the Most English Speakers?" K International. http://www.k-international.com/blog/countries-with-the-most-english-speakers/.

Kumar, Shamanth, Geoffrey Barbier, Mohammad Ali Abbasi, and Huan Liu. 2011. "TweetTracker: An Analysis Tool for Humanitarian and Disaster Relief." Proceedings of the Fifth International AAAI Conference on Weblogs and Social Media.

Kumar, Shamanth, Fred Morstatter, and Huan Liu. 2014. "Monitoring Social Media for Humanitarian Assistance and Disaster Relief." In Hermann Hellwagner, Daniela Pohl, and Rene Kaiser (eds.), "Social Media Analysis for Crisis Management." IEEE Computer Society Special Technical Community on Social Networking E-Letter 2(1).

Legara, E. 2015. "Urbanism in the Philippines: A Different Look at the Philippine Map." $A$ Byte of my 22-lb Brain. https://erikafille.ph/2015/09/10/urbanism-in-the-philippines/.

Liu, Bing, Minqing Hu, and Junsheng Cheng. 2005. "Opinion Observer: Analyzing and Comparing Opinions on the Web.” Proceedings of the 14th International World Wide Web Conference ( $W W W$-2005). May 10-14, 2005, Chiba, Japan. 
MacLean, Jessica. 2015. "Can Standardised Hashtags Be Effective in Emergency Responses?” EISF. https://www.eisf.eu/news/can-standardised-hashtags-be-effective-inemergency-responses/.

Mahmud, Jalal, Jeffrey Nichols, and Clemens Drews. 2012. Home Location Identification of Twitter Users. IBM Research - Almaden. https://arxiv.org/abs/1403.2345v1.

Meier, Patrick. 2012. "How Crisis Mapping Saved Lives in Haiti." National Geographic. 2 Jul 2012. http://voices.nationalgeographic.org/2012/07/02/crisis-mapping-haiti/.

Morstatter, Fred, Jurgen Pfeffer, Huan Liu, and Kathleen M. Carley. 2013. "Is the Sample Good Enough? Comparing Data from Twitter's Streaming API with Twitter's Firehose." Proceedings of the Seventh International AAAI Conference on Weblogs and Social Media. https://www.aaai.org/ocs/index.php/ICWSM/ICWSM13/paper/viewFile/6071/6379.

Ozdikis, Ozer, Halit Oguztuzun, and Pinar Karagoz. 2017. "A Survey of Location Estimation Techniques for Events Detected in Twitter.” Knowledge Information Systems 52:291-339.

Paraskevopoulos, Pavlos, and Temis Palpanas. 2016. "Where Has this Tweet Come from? Fast and Fine-Grained Geolocalization of Non-Geotagged Tweets." Social Network Analysis and Modeling. 6:89. https://doi.org/10.1007/s13278-016-0400-7.

Poulston, Adam, Mark Stevenson, and Kalina Bontcheva. 2017. "Hyperlocal Home Location Identification of Twitter Profiles." HT '17 Proceedings of the 28th ACM Conference on Hypertext and Social Media. pp 45-54. https://doi.org/10.1145/3078714.3078719.

Roller, Stephen, Michael Speriosu, Sarat Rallapalli, Benjamin Wing, and Jason Baldridge. 2012. "Supervised Text-based Geolocation Using Language Models on an Adaptive Grid." Proceedings of the 2012 Joint Conference on Empirical Methods in Natural Language Processing and Computational Natural Language Learning.

Ruth, Derek, and Jurgen Pfeffer. 2014. "Social Media for Large Studies of Behavior." Science Magazine 346:6213, pp 1063-1064.

Sadilek, Adam, Henry Kautz, and Jeffrey P. Bingham. 2012. "Finding Your Friends and Following Them to Where You Are." WSDM '12. https://pdfs.semanticscholar.org/57d7/6d5716a0007e0fbf451866bf665887628613.pdf.

Salathe, Marcel, and Shashank Khandelwal. 2011. "Assessing Vaccination Sentiments with Online Social Media: Implications for Infectious Disease Dynamics and Control.” PLoS Computational Biology. https://doi.org/10.1371/journal.pcbi.1002199.

Schulz, Axel, Aristotelis Hadjakos, Heiko Paulheim, Johannes Nachtway, Max Muhlhauser. 2013. "A Multi-Indicator Approach for Geolocalization of Tweets." "Proceedings of the Seventh International AAAI Conference on Weblogs and Social Media."

Shelton, Taylor, Ate Poorthuis, and Matthew Zook. 2015. "Social Media and the City: Rethinking Urban Socio-Spatial Inequality Using User-Generated Geographic Information." Landscape and Urban Planning, Forthcoming . https://ssrn.com/abstract=2571757 or http://dx.doi.org/10.2139/ssrn.2571757. 
Shelton, Taylor. 2017. "Spatialities of Data: Mapping Social Media 'Beyond the Geotag'." GeoJournal 82:4, pp 721-734.

Steiger, Enrico, Joao Porto de Albuquerque, and Alexander Zipf. 2015. "An Advanced Systematic Literature Review on Spatiotemporal Analyses of Twitter Data." Transactions in GIS. 19:809-834. doi:10.1111/tgis.12132.

Szomszor, Martin, Patty Kostkova, and Ed de Quincey. 2010. “\#Swineflu: Twitter Predicts Swine Flu Outbreak in 2009.” In M. Szomszor and P. Kostkova (eds) Electronic Healthcare. eHealth 2010. Lecture Notes of the Institute for Computer Sciences, Social Informatics and Telecommunications Engineering, vol 69. Springer, Berlin, Heidelberg.

Third Team Media. 2017. The State of Social Media and Digital in the Philippines for 2017. https://www.slideshare.net/likke13/the-state-of-social-media-and-digital-in-thephilippines-for-2017.

Twitter. 2017. "Search Tweets." Twitter Developer. https://developer.twitter.com/en/docs/tweets/search/overview.

USAID (U.S. Agency for International Development). 2011. "Demographic and Health Surveys Methodology.” Questionnaires: Household, Woman’s, and Man’s. 2016. StatCompiler - The DHS Program. http://statcompiler.com/en/.

U.S. Department of State. 2015. "Bureau of Democracy, Human Rights, and Labor." Country Reports on Human Rights Practices for 2015. Bangladesh. https://www.state.gov/i/drl/rls/hrrpt/humanrightsreport/index.htm?dlid=252821\&year=2015.

Wagner, Kurt. 2017. "How Many People Use Twitter Every Day?” Recode. https://www.recode.net/2017/7/27/16049334/twitter-daily-active-users-dau-growth-q2earnings-2017.

Wing, Benjamin P., and Jason Baldridge. 2011. "Simple Supervised Document Geolocation with Geodesic Grids." Proceedings of the 49th Annual Meeting of the Association for Computational Linguistics, pp 955-964. http://www.aclweb.org/anthology/P11-1096. 


\section{Appendix A: Social Media Use (Twitter) Framework Technical Documentation}

\section{A.1 Social media user framework overview}

The Social Media User framework is a computational framework that seeks to identify likely regular users of social media in the Philippines (with particular emphasis on the metropolitan Manila area). While this framework is structured and evaluated in a way that pinpoints social media use in general (i.e., it is not platform specific), the goal of this framework is to augment an open-source Twitter model to reduce uncertainty of both social mediums posting content, location, and sentiment.

This framework, in its current state, consists mostly of metrics from publicly available international surveys. The conditions, factors, and indicator descriptions are inclusive enough where other data sources can augment the framework component in a way that can further reduce the uncertainty across all levels.

Conditions within this framework include Population, Technology, and Place.

\section{A.1.1 Condition weighting logic}

All three conditions within the Social Media Framework are given both a minimum and a maximum value of 0.333 . This is because none of the conditions will, by themselves, determine the framework's risk value.

\begin{tabular}{|l|c|c|}
\hline Condition (Heading Number) & Weight Minimum Value & Weight Maximum Value \\
\hline Population (A2) & 0.333 & 0.333 \\
\hline Technology (A3) & 0.333 & 0.333 \\
\hline Place (A4) & 0.333 & 0.333 \\
\hline
\end{tabular}




\section{A.1.2 Example outcome}

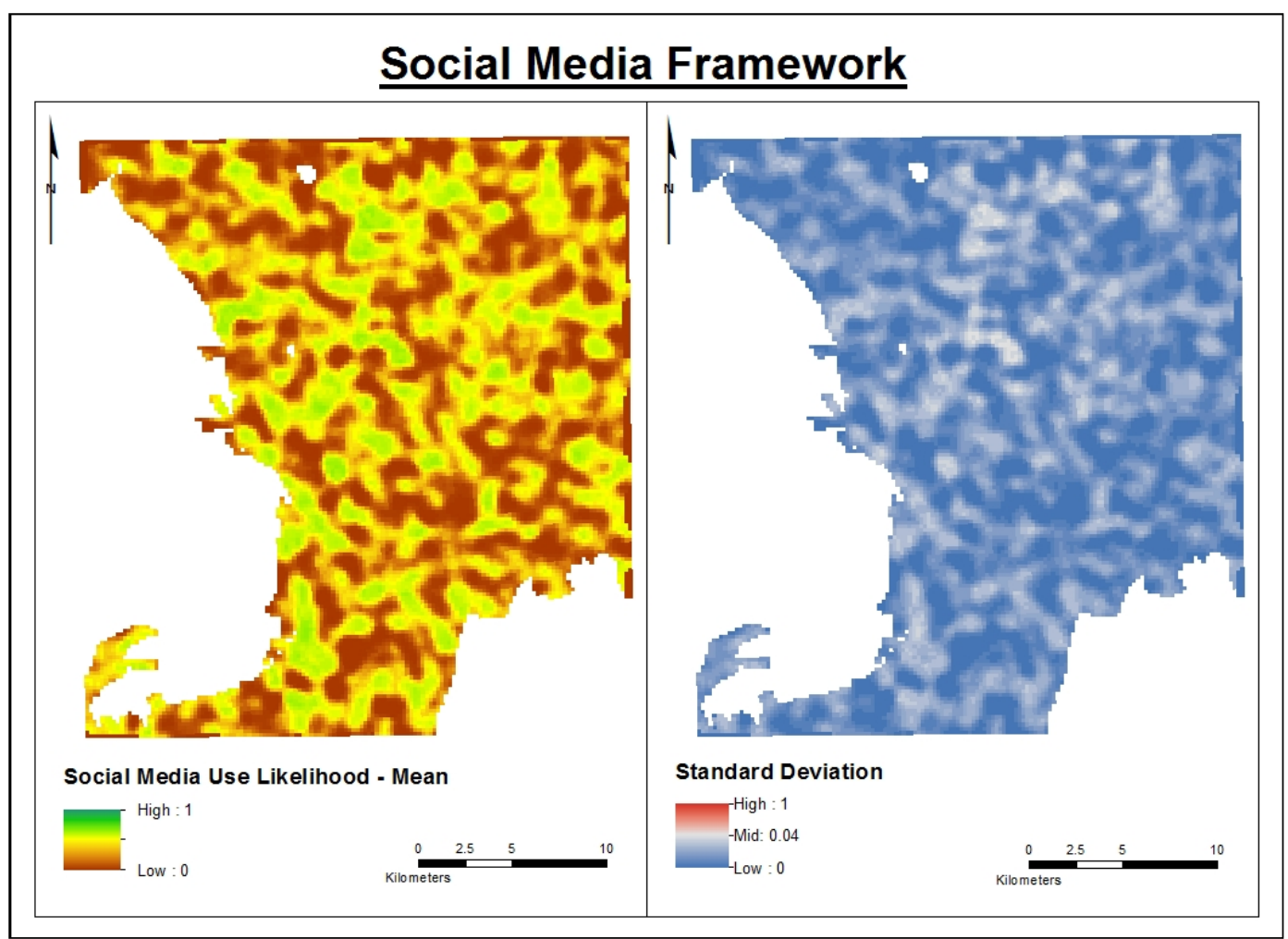

\section{A.2 Population condition overview}

This condition encompasses the population demographic aspects of likely social media users. In essence, this condition accounts for the characteristics of persons (Age, Gender, Educational Level) that increases or decreases the likelihood of them using social media.

All factors currently have available metric and indicator data. Factors within this condition include Individual Characteristics and Education.

\section{A.2.1 Factor weighting logic}

Both the Individual Characteristics and Education factors are weighted a minimum and maximum of 0.5 , as they are equally important in predicting the likelihood social media use.

\begin{tabular}{|l|c|c|}
\hline Factor & Weight Minimum Value & Weight Maximum Value \\
\hline Individual Characteristics & 0.5 & 0.5 \\
\hline Education & 0.5 & 0.5 \\
\hline
\end{tabular}




\section{A.2.2 Uncertainty values}

This condition is given a range of uncertainty values from 0.1 to 0.3 because of the perceived ability of the existing factors to fully address the themes of the condition. As more research on social media users, particularly social media/Twitter users in the Philippines, additional demographic factors could further reduce the uncertainty.

\begin{tabular}{|l|l|}
\hline Condition Uncertainty Min: & 0.1 \\
\hline Condition Uncertainty Max: & 0.3 \\
\hline
\end{tabular}

\section{A.2.3 Example outcome}

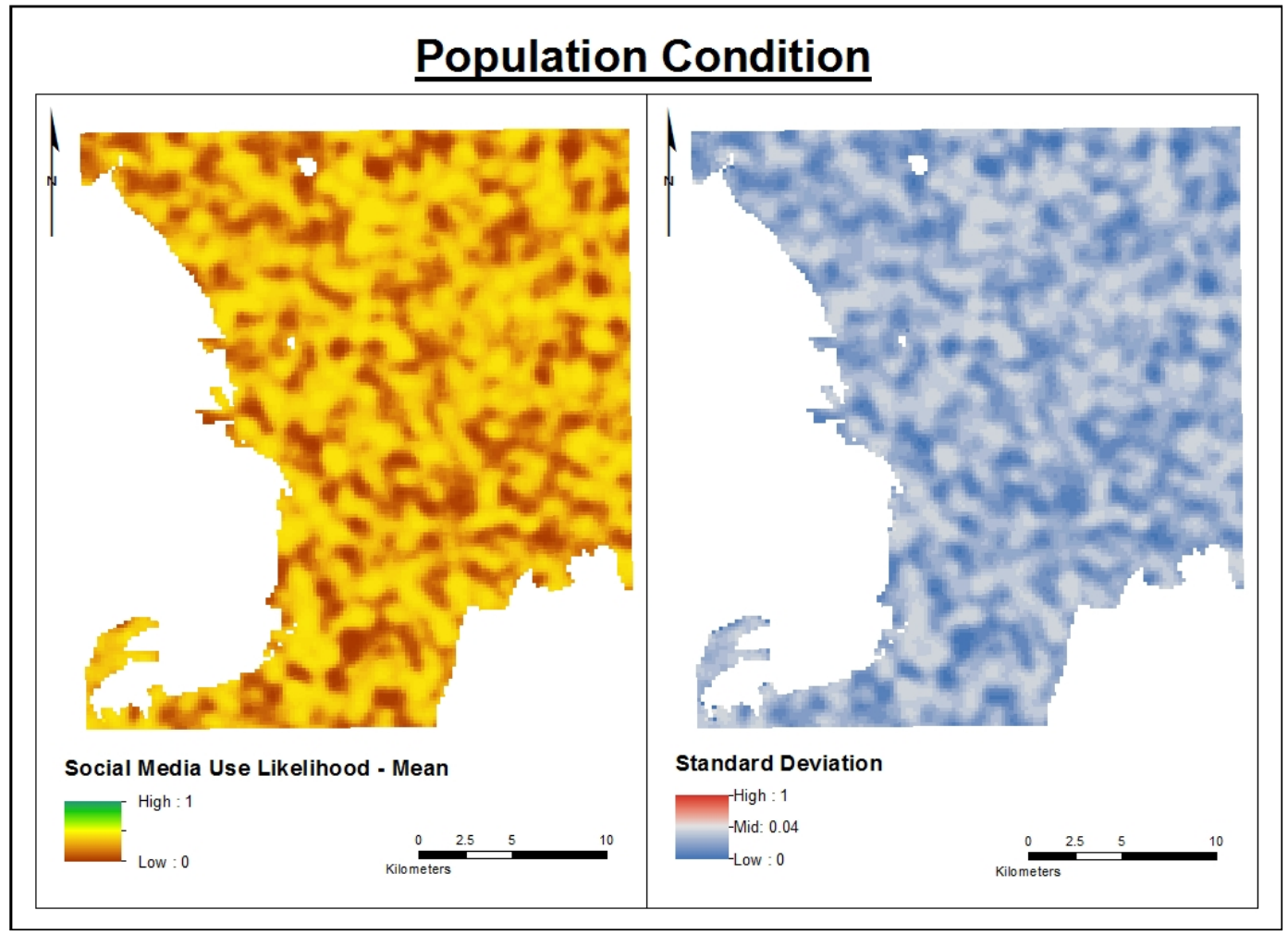

\section{A.3 Individual characteristics factor overview}

The Individual Characteristics Factor focuses on immutable personal traits of individuals represented in the sample population. All indicators currently have available metric data.

Indicators within this factor include Sex \& Gender, Age, and Disability. 


\section{A.3.1 Indicator weighting logic}

As an individual with a disability - particularly a vision impairment would most likely preclude the likelihood of someone being a regular social media user, the Disability indicator is given the highest maximum weight value. Age is more of a determinant than Sex/Gender, giving the Age indicator a higher minimum and maximum weight.

\begin{tabular}{|l|c|c|}
\hline Indicator & Weight Minimum Value & Weight Maximum Value \\
\hline Sex \& Gender & 0.4 & 0.6 \\
\hline Age & 0.6 & 0.8 \\
\hline Disability & 0.6 & 0.9 \\
\hline
\end{tabular}

\section{A.1.1 Uncertainty values}

This factor is given a range of uncertainty values from 0.1 to 0.3 because of the perceived ability of the existing indicators to fully address the themes of the factor. As more research on social media users, particularly social media/Twitter users in the Philippines, additional demographic indicators could further reduce the uncertainty.

\begin{tabular}{|l|l|}
\hline Factor Uncertainty Min: & 0.1 \\
\hline Factor Uncertainty Max: & 0.3 \\
\hline
\end{tabular}




\section{A.1.2 Example outcome}

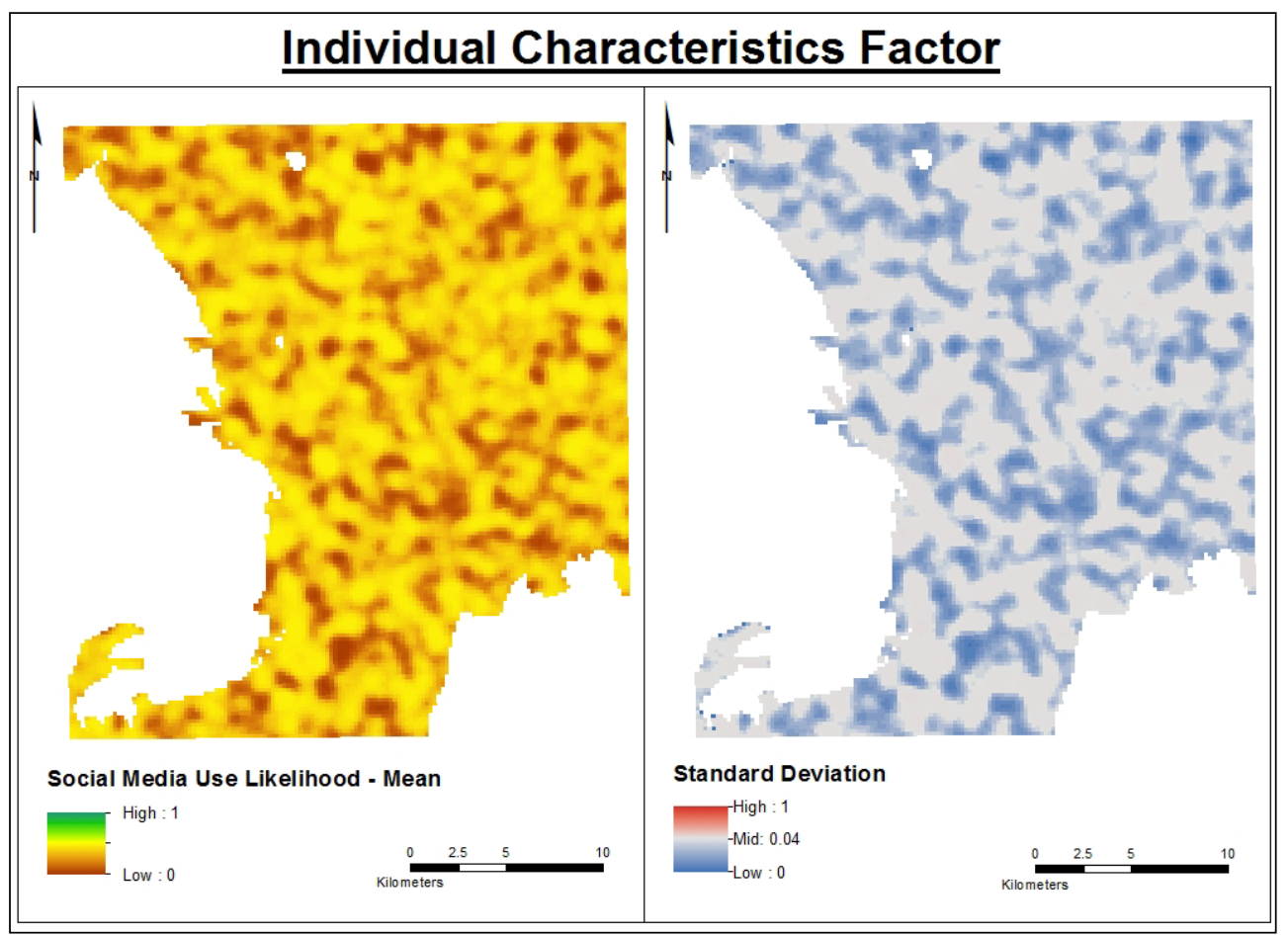

\section{A.1.3 Indicator: Sex \& gender}

The Sex and Gender indicator accounts for the gender characteristics individuals represented in the sample population.

\section{A.1.4 Metric weighting logic}

As both metrics within this indicator represent the same kind of data, they are weighted the same and given both a minimum and maximum value of 0.5 .

\begin{tabular}{|l|c|c|}
\hline Metric & Weight Minimum Value & Weight Maximum Value \\
\hline DHSSex & 0.5 & 0.5 \\
\hline IPUMSSex & 0.5 & 0.5 \\
\hline
\end{tabular}

\section{A.1.5 Uncertainty values}

This indicator is given an uncertainty minimum value of 0.1 and an uncertainty maximum value of 0.2 because of the perceived ability of the existing metrics to fully address the themes of the indicator.

\begin{tabular}{|l|l|}
\hline Indicator Uncertainty Min: & 0.1 \\
\hline Indicator Uncertainty Max: & 0.2 \\
\hline
\end{tabular}




\section{A.1.6 Example outcome}

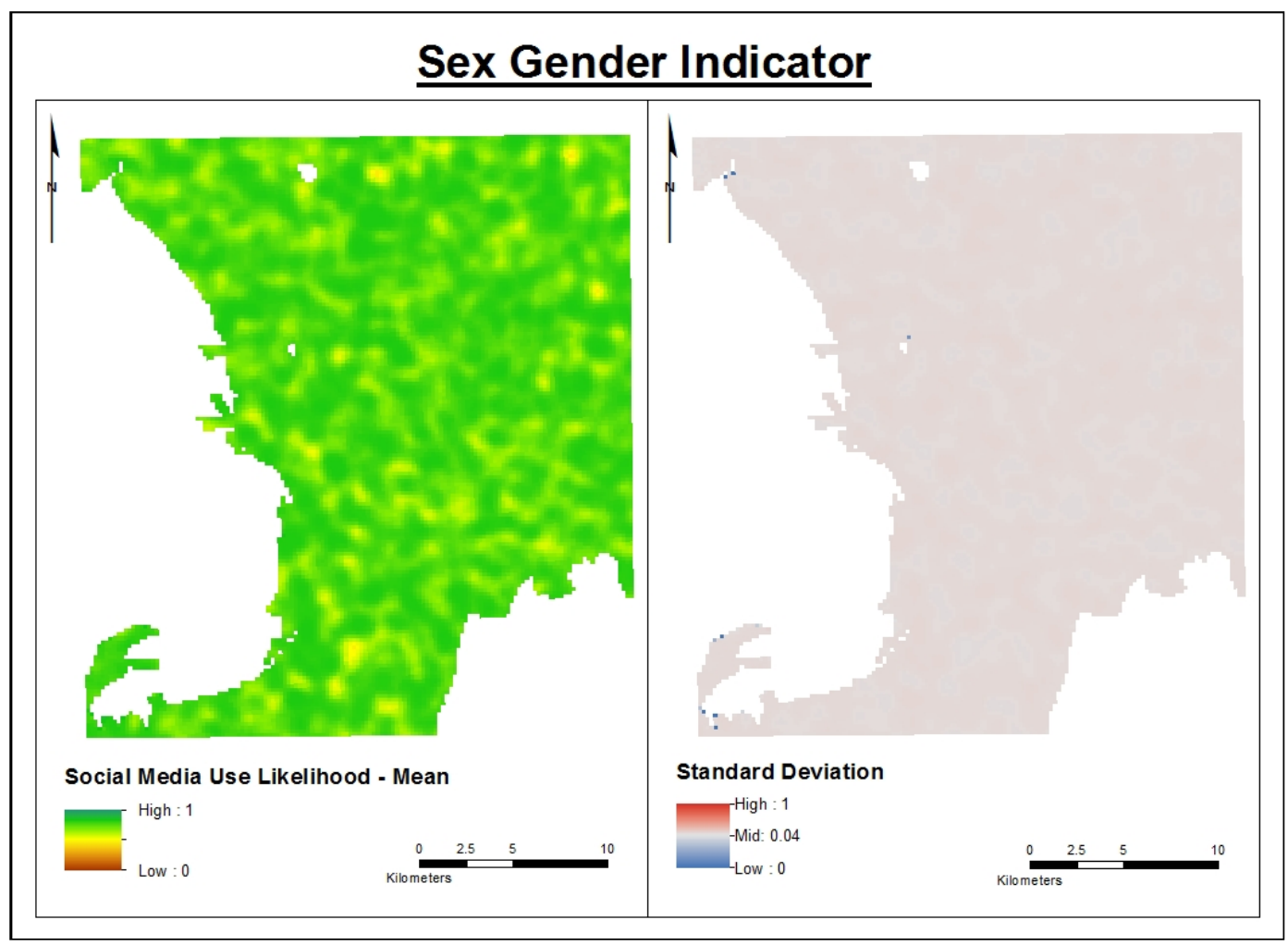

\section{A.1.7 Metric: DHSSex}

\begin{tabular}{|l|l|}
\hline Indicator: & Sex \& Gender \\
\hline Factor: & Individual Characteristics \\
\hline Condition: & Population \\
\hline Framework: & Social Media User \\
\hline $\begin{array}{l}\text { Metric Assigned } \\
\text { Values: }\end{array}$ & $\begin{array}{l}\text { Male (min: 0.5, max: 1.0) } \\
\text { Female (min: 0.5, max: 0.9) }\end{array}$ \\
\hline Survey: & DHS \\
\hline Survey Date: & 2013 \\
\hline $\begin{array}{l}\text { Other Data } \\
\text { Sources: }\end{array}$ & Third Team Media, 2017; Greenwood et al, 2016 \\
\hline Logic: & $\begin{array}{l}\text { This metric consists of survey respondent gender identification from the } \\
\text { DHS survey. Answers of “Male" are given a minimum value of 0.5 (slight } \\
\text { risk, } 1 / 2^{1} \text { ) and a maximum value of 1.0 (minimal risk with no impact on } \\
\left.\text { risk, } 1 / 2^{0}\right) . \text { Answers of “Female" are given a minimum value of 0.5 and a } \\
\text { maximum value of 0.9 (minimal risk). } \\
\text { According to Third Team Media, men are slightly more likely to be social } \\
\text { media users than women (2017). In an American context, men and } \\
\text { women used Twitter at equal percents (Greenwood et al, 2016). } \\
\text { Maps will range from slight risk to no risk. }\end{array}$ \\
\hline Example Realization Metric Map \\
\hline
\end{tabular}




\section{A.1.8 References}

Third Team Media. The State of Social Media and Digital in the Philippines for 2017. Jan 24, 2017. https://www.slideshare.net/likke13/the-state-of-social-media-and-digital-inthe-philippines-for-2017 on 8 Nov 2017.

Greenwood, Shannon, Andrew Perrin and Maeve Duggan. Social Media Update 2016. Pew Research Center. Nov 11, 2016. http://www.pewinternet.org/2016/11/11/socialmedia-update-2016/.

\section{A.1.9 Metric: IPUMSSex}

\begin{tabular}{|c|c|}
\hline Indicator: & Sex \& Gender \\
\hline Factor: & Individual Characteristics \\
\hline Condition: & Population \\
\hline Framework: & Social Media User \\
\hline $\begin{array}{l}\text { Metric Assigned } \\
\text { Values: }\end{array}$ & $\begin{array}{l}\text { Male (min: 0.5, max: } 1.0) \\
\text { Female (min: 0.5, max: 0.9) }\end{array}$ \\
\hline Survey: & IPUMS \\
\hline Survey Date: & 2000 \\
\hline $\begin{array}{l}\text { Other Data } \\
\text { Sources: }\end{array}$ & Third Team Media, 2017; Greenwood et al, 2016 \\
\hline Logic: & $\begin{array}{l}\text { This metric consists of survey respondent gender identification from } \\
\text { the IPUMS survey. Answers of "Male" are given a minimum value of } \\
0.5 \text { (slight risk, } 1 / 2^{1} \text { ) and a maximum value of } 1.0 \text { (minimal risk with no } \\
\text { impact on risk, } 1 / 2^{0} \text { ). Answers of "Female" are given a minimum value } \\
\text { of } 0.5 \text { and a maximum value of } 0.9 \text { (minimal risk). }\end{array}$ \\
\hline & $\begin{array}{l}\text { According to Third Team Media, men are slightly more likely to be } \\
\text { social media users than women (2017). In an American context, men } \\
\text { and women used Twitter at equal percents (Greenwood et al, 2016). } \\
\text { Maps will range from slight risk to no risk. }\end{array}$ \\
\hline
\end{tabular}

Example Realization Metric Map 


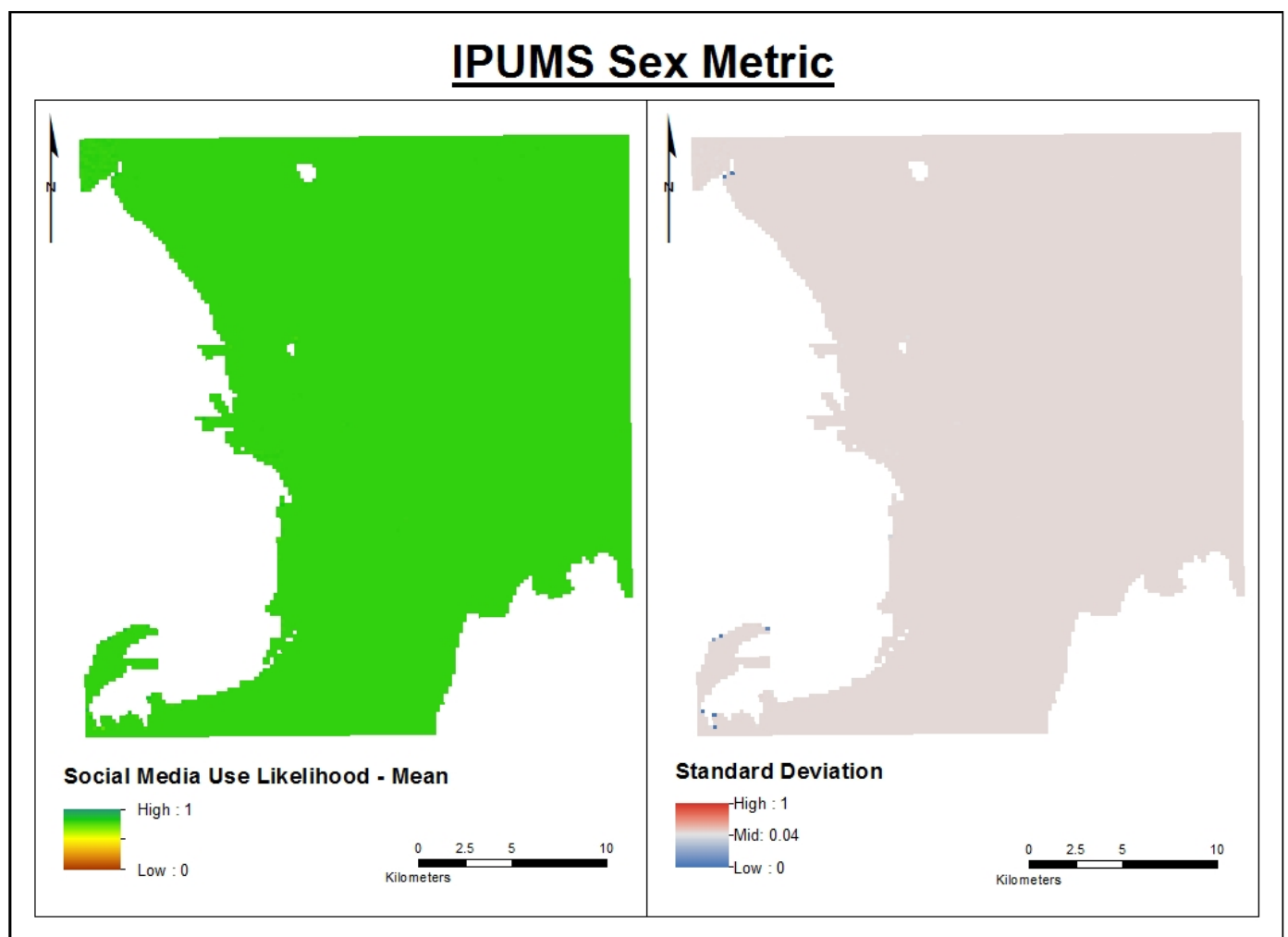

\section{A.1.10 References}

Third Team Media. The State of Social Media and Digital in the Philippines for 2017. Jan 24, 2017. https://www.slideshare.net/likke13/the-state-of-social-media-and-digital-inthe-philippines-for-2017 on 8 Nov 2017.

Greenwood, Shannon, Andrew Perrin and Maeve Duggan. Social Media Update 2016. Pew Research Center. Nov 11, 2016. http://www.pewinternet.org/2016/11/11/socialmedia-update-2016/.

\section{A.1.11 Indicator: Age}

The Age indicator accounts for the age characteristics individuals represented in the sample population.

\section{A.1.12 Metric weighting logic}

As there is only one metric for this indicator, it is given both a minimum and maximum value of 1.0.

\begin{tabular}{|l|c|c|}
\hline Metric & Weight Minimum Value & Weight Maximum Value \\
\hline IPUMSAge & 1.0 & 1.0 \\
\hline
\end{tabular}




\section{A.1.13 Uncertainty values}

This indicator is given an uncertainty minimum value of 0.1 and an uncertainty maximum value of 0.2 because of the perceived ability of the existing metrics to fully address the themes of the indicator. The addition of metrics measuring age groups of Twitter users specifically could further reduce the uncertainty values.

\begin{tabular}{|l|l|}
\hline Indicator Uncertainty Min: & 0.1 \\
\hline Indicator Uncertainty Max: & 0.2 \\
\hline
\end{tabular}

\section{A.1.14 Example outcome}

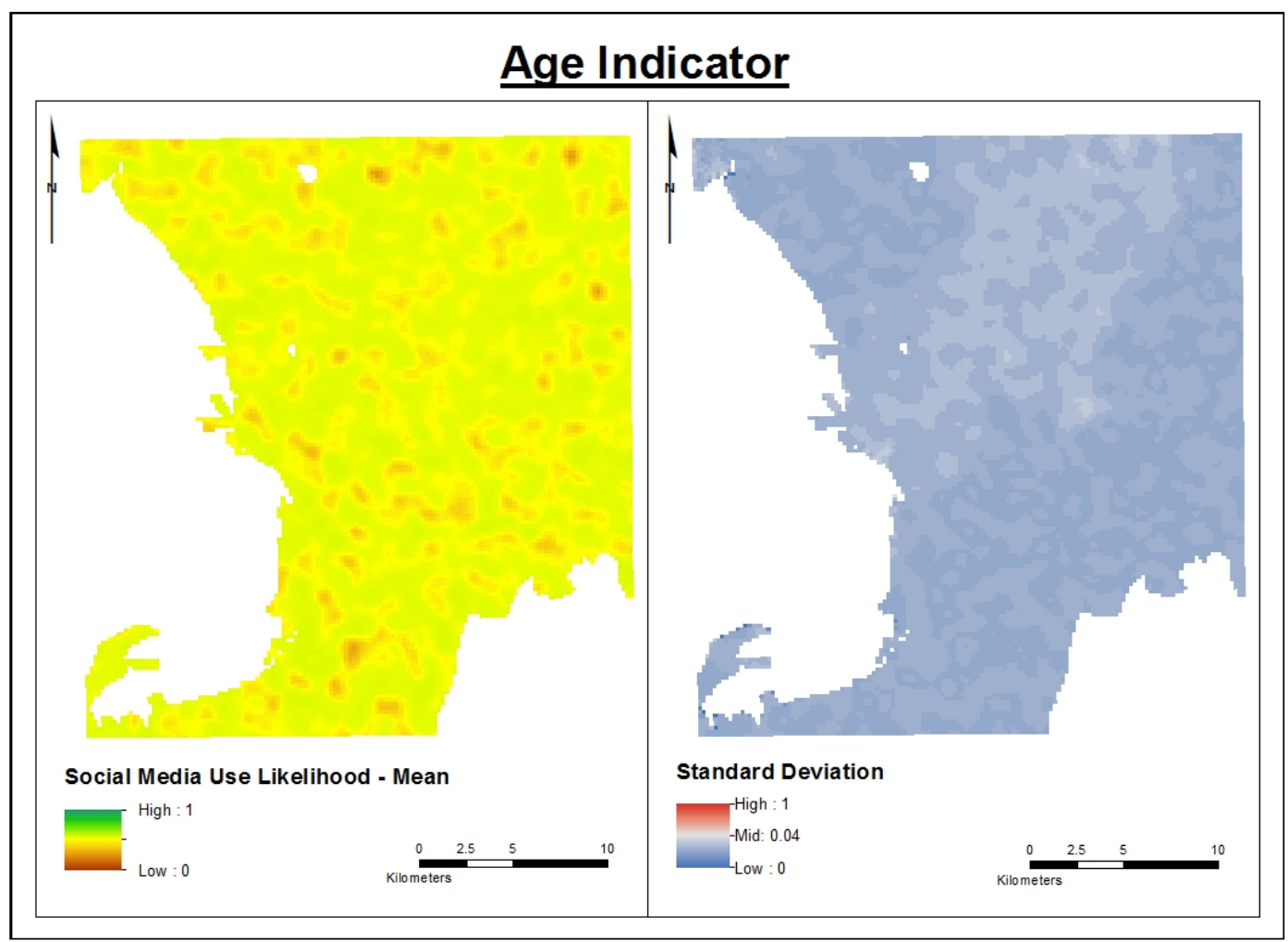

\section{A.1.15 Metric: IPUMSAge}

$\begin{array}{ll}\text { Indicator: } & \text { Age } \\ \text { Factor: } & \text { Individual Characteristics } \\ \text { Condition: } & \text { Population } \\ \text { Framework: } & \text { Social Media User }\end{array}$


Metric Assigned Values: 0to12 (min: 0.001, max: 0.0625)

13 to17 (min: 0.25, max: 0.6)

18to24 (min: 0.5, max: 0.9)

25to34 (min: 0.5, max: 0.8)

35to44 (min: 0.25, max: 0.6)

45to54 (min: 0.125, max: 0.25)

55to64 (min: 0.01, max: 0.125)

65+ (min: 0.001, max: 0.0625)

Survey: IPUMS

Survey Date: 2000

Other Data Sources: Third Team Media, 2017

Logic:

This metric consists of respondent and household member age from the IPUMS survey. Answers of 0 to 12 years and 65+ years are given a minimum value of 0.001 (extreme risk) and a maximum value of 0.0625 (extreme risk, $1 / 2^{4}$ ). Answers of 13 to 17 and 35 to 44 are given a minimum value of 0.25 (medium risk, $1 / 2^{2}$ ) and a maximum value of 0.6 (minimal risk). Answers of 18 to 24 are given a minimum value of 0.5 (slight risk, $1 / 2^{1}$ ) and a maximum value of 0.9 (minimal risk). Answers of 25 to 34 are given a minimum value of 0.5 and a maximum value of 0.8 (minimal risk). Answers of 45 to 54 are given a minimum value of 0.125 (high risk, $1 / 2^{3}$ ) and a maximum value of 0.25 . Answers of 55 to 64 are given a minimum value of 0.01 (extreme risk) and a maximum value of 0.125 .

Third Team Media ranked Facebook users by age in the Philippines in 2017. These age rankings were used to evaluate the IPUMS survey answers.

Maps will range from extreme risk to minimal risk.

Example Realization Metric Map 


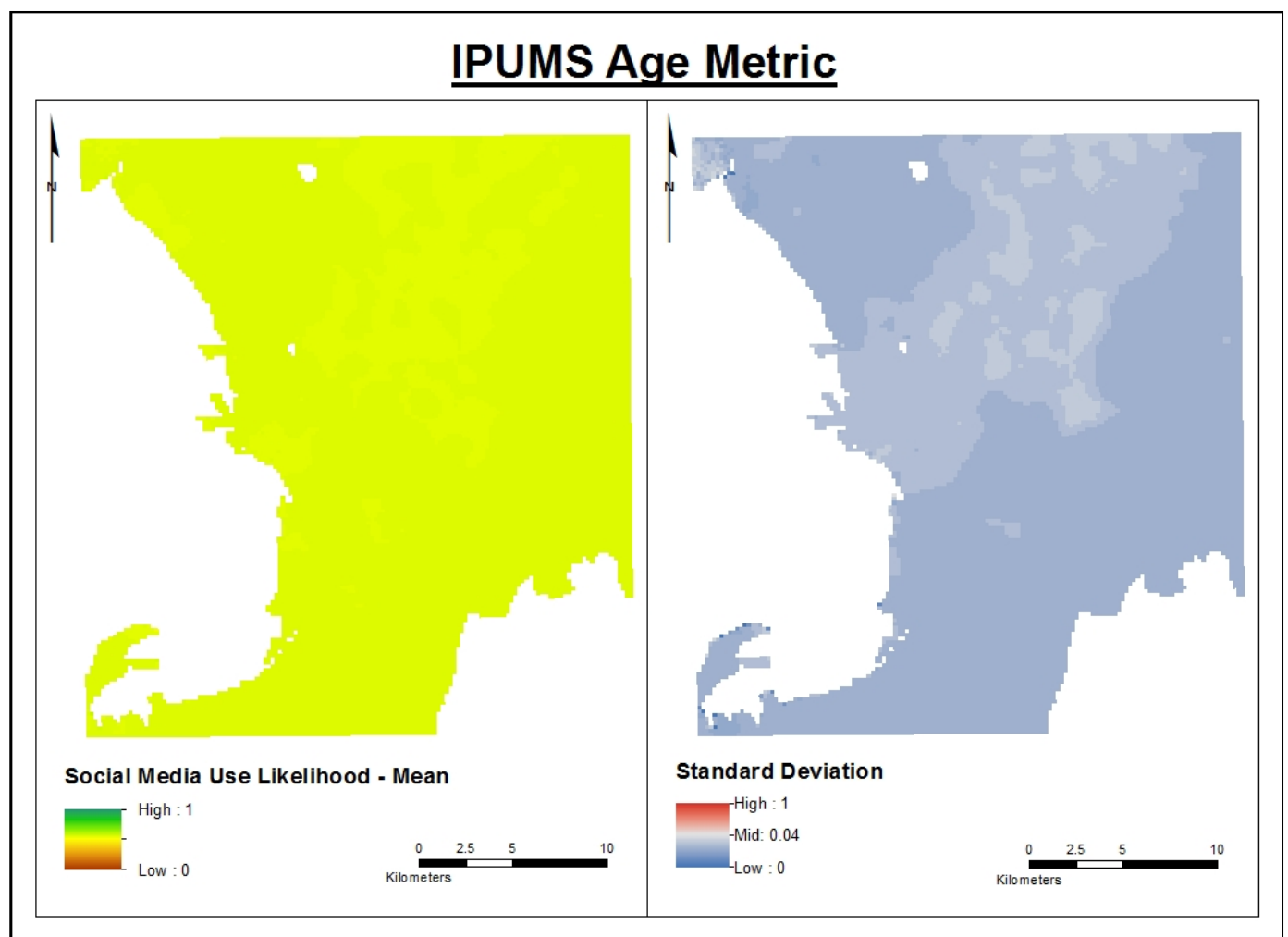

\section{A.1.16 References}

Third Team Media. The State of Social Media and Digital in the Philippines for 2017. Jan 24, 2017. https://www.slideshare.net/likke13/the-state-of-social-media-and-digital-inthe-philippines-for-2017.

\section{A.1.17 Indicator: disability}

The Disability indicator accounts for whether or not individuals in the sample population have a disability that could potentially impact their use of social media.

\section{A.1.18 Metric weighting logic}

As the DisabilityStatus metric is more general, it is weighted lower than the VisionImpaired metric.

\begin{tabular}{|l|c|c|}
\hline Metric & Weight Minimum Value & Weight Maximum Value \\
\hline Visionlmpaired & 0.6 & 0.8 \\
\hline DisabilityStatus & 0.5 & 0.6 \\
\hline
\end{tabular}




\section{A.1.19 Uncertainty values}

This indicator is given an uncertainty minimum value of 0.1 and an uncertainty maximum value of 0.3 because of the perceived ability of the existing metrics to fully address the themes of the indicator.

\begin{tabular}{|l|l|}
\hline Indicator Uncertainty Min: & 0.1 \\
\hline Indicator Uncertainty Max: & 0.3 \\
\hline
\end{tabular}

\section{A.1.20 Example outcome}

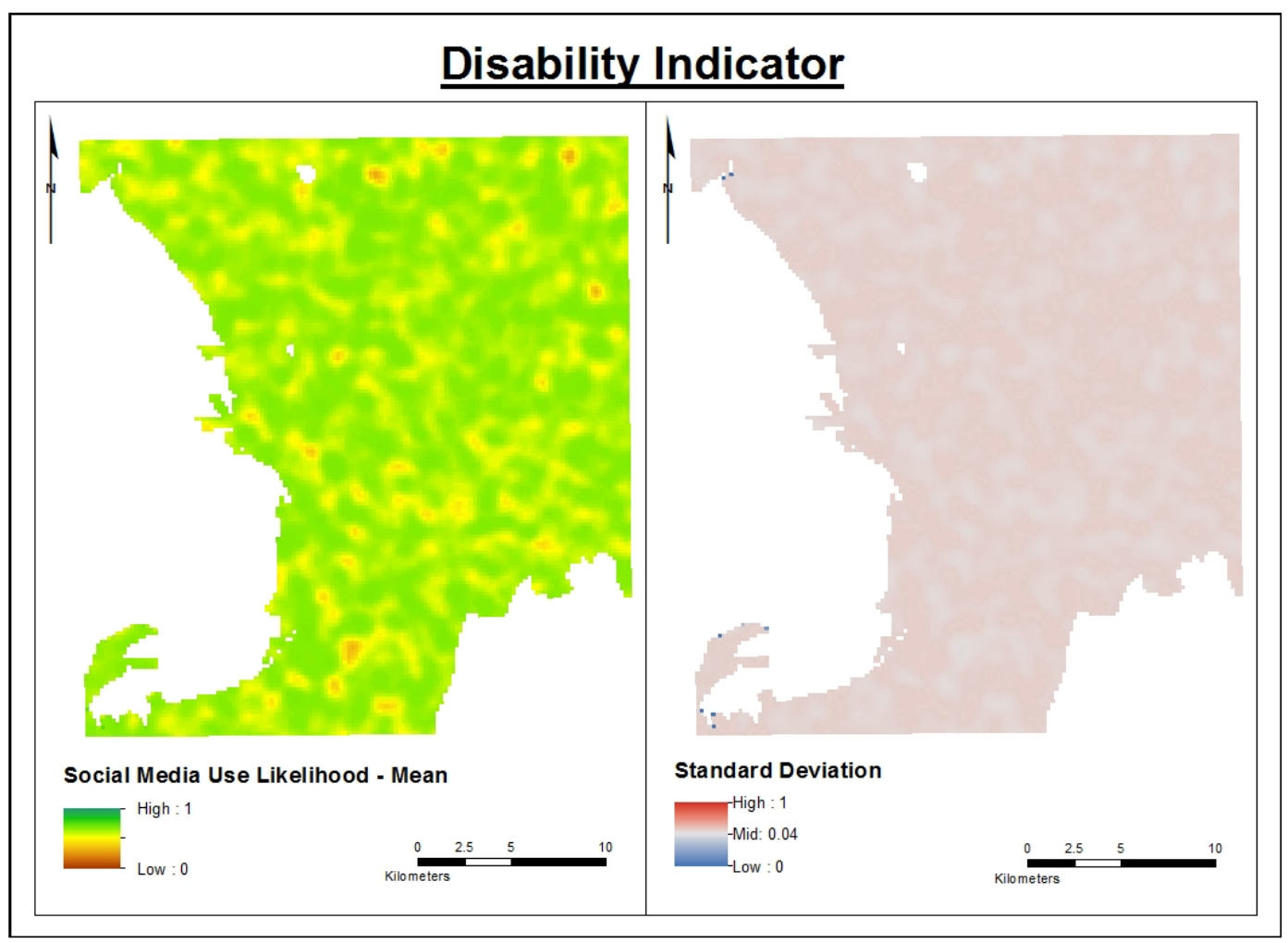

\section{A.1.21 Metric: VisionImpaired}

$\begin{array}{ll}\text { Indicator: } & \text { Disability } \\ \text { Factor: } & \text { Individual Characteristics } \\ \text { Condition: } & \text { Population } \\ \text { Framework: } & \text { Social Media User } \\ \text { Metric Assigned } & \text { Yes (min: 0.125, max: 0.25) } \\ \text { Values: } & \text { No (min: 0.25, max: 0.9) } \\ & \text { Unknown (min: 0.125, max: 0.9) } \\ \text { Survey: } & \text { IPUMS } \\ \text { Survey Date: } & 2000\end{array}$


Other Data Sources: N/A

Logic:

This metric consists of answers to the question on whether or not the respondent has any kind of vision impairment and/or are blind from the IPUMS survey. Answers of "Yes" are given a minimum value of 0.125 (high risk, $1 / 2^{3}$ ) and a maximum value of 0.25 (medium risk, $1 / 2^{2}$ ). Answers of "No" are given a minimum value of 0.25 and a maximum value of 0.9 (minimal risk). Answers of "Unknown" are given a minimum value of 0.125 and a maximum value of 0.9. Maps will range from high risk to minimal risk.

Example Realization Metric Map

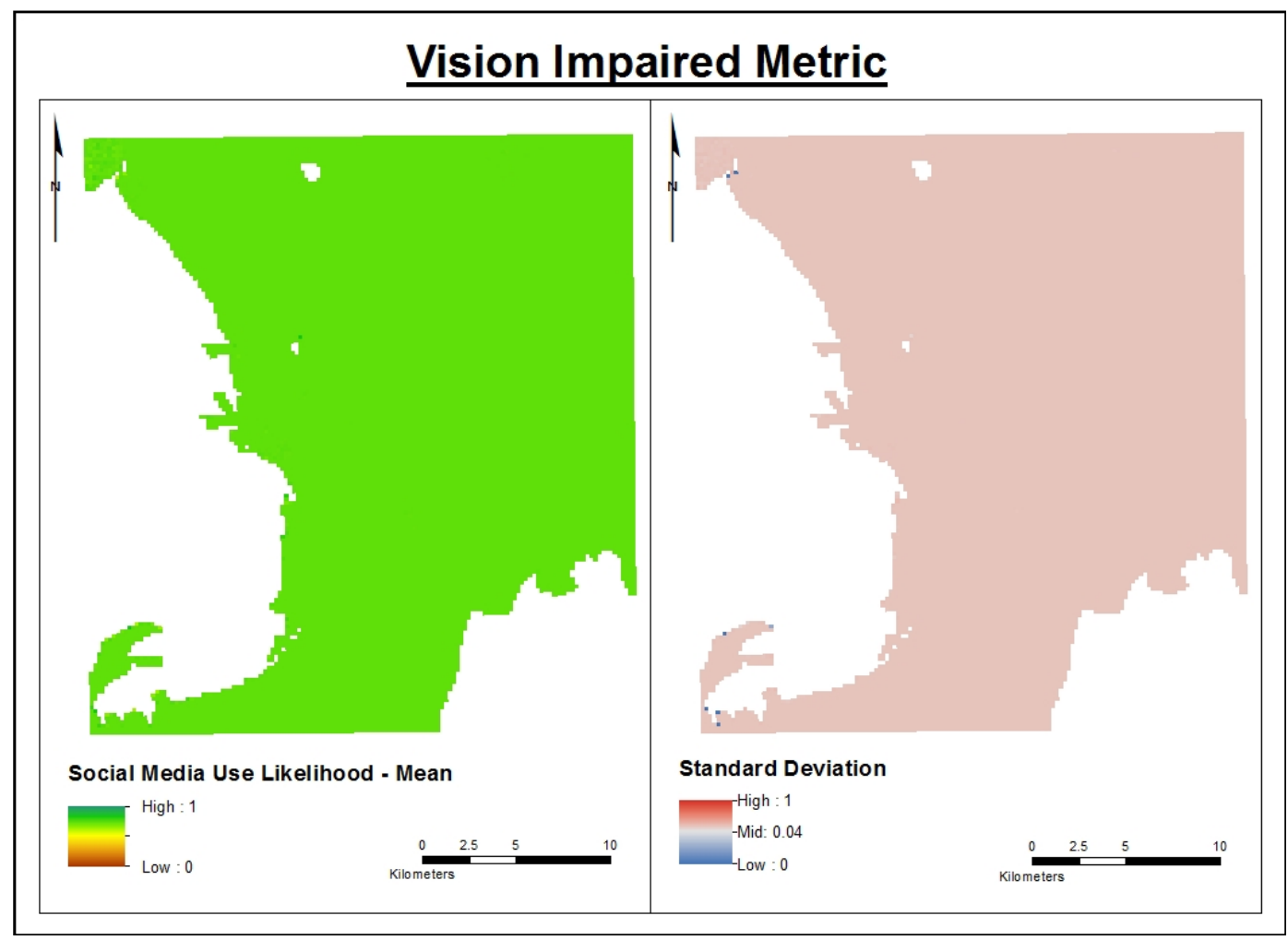

\section{A.1.22 Metric: DisabilityStatus}

Indicator:

Disability

Factor:

Individual Characteristics

Condition:

Population

Framework:

Social Media User

Metric Assigned

Yes (disabled) (min: 0.125, max: 0.5)

Values:

No (not disabled) (min: 0.25, max: 0.9)

Unknown (min: 0.125, max: 0.9)

Survey: IPUMS

Survey Date: 2000

Other Data Sources: N/A 
Logic:

This metric consists of answers to the question of whether the respondent has any kind of disability from the IPUMS survey. Answers of "Yes" are given a minimum value of 0.125 (high risk, $1 / 2^{3}$ ) and a maximum value of 0.25 (medium risk, $1 / 2^{2}$ ). Answers of "No" are given a minimum value of 0.25 and a maximum value of 0.9 (minimal risk). Answers of "Unknown" are given a minimum value of 0.125 and a maximum value of 0.9 . Maps will range from high risk to minimal risk.

Example Realization Metric Map

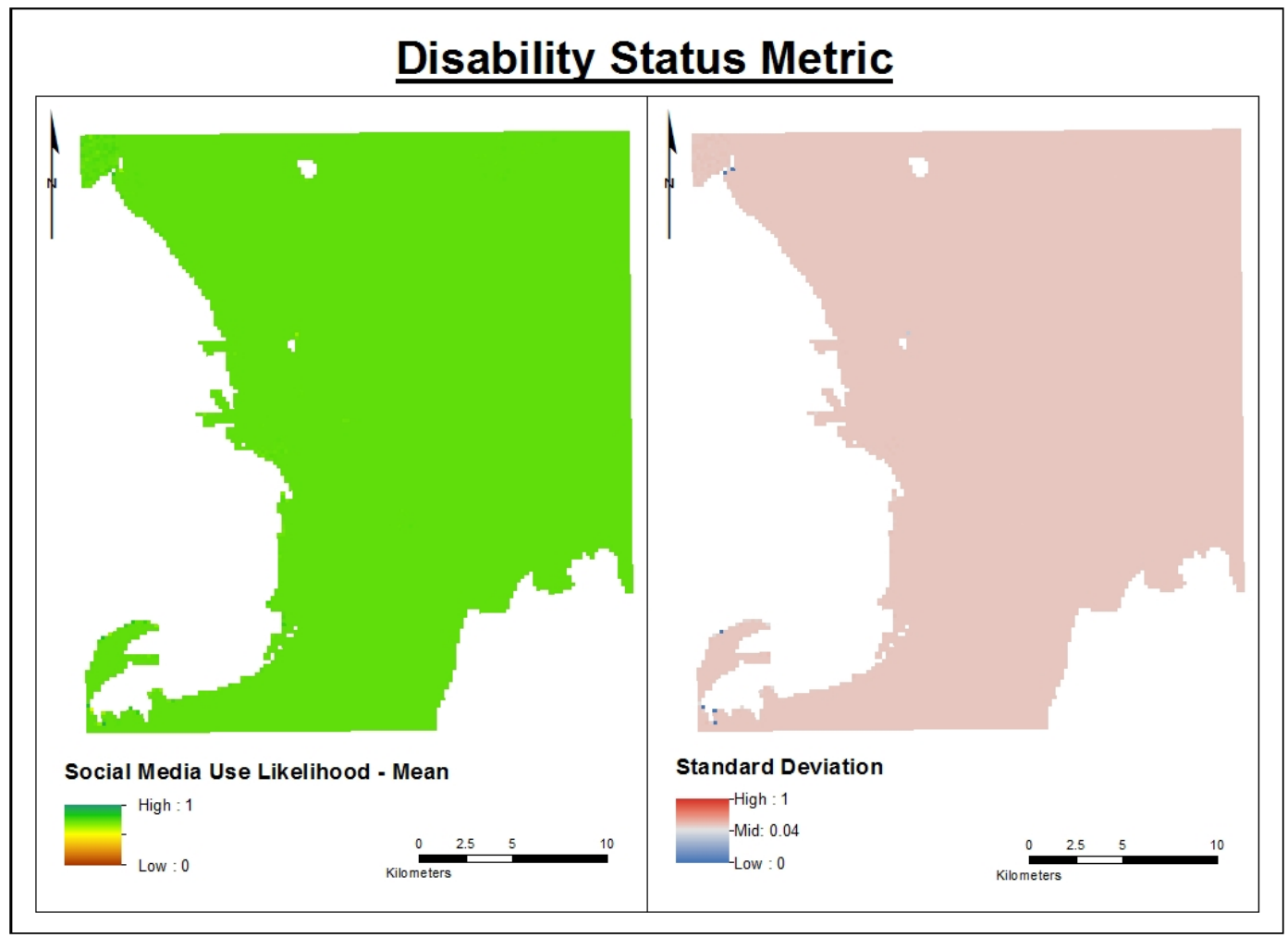

\section{A.2 Education factor overview}

This factor concerns the educational backgrounds of the sample population. Indicators within this factor include Language Abilities, Literacy, and Education Attainment. All indicators currently have available metric data.

\section{A.2.1 Indicator weighting logic}

As literacy and higher educational attainment has been shown to be indicators of higher developments in ICT (information and communication technologies), which include social media platforms, these metrics are weighted higher than Language Abilities (Albert et al. 2016). 


\begin{tabular}{|l|c|c|}
\hline Indicator & Weight Minimum Value & Weight Maximum Value \\
\hline Language Abilities & 0.3 & 0.5 \\
\hline Literacy & 0.5 & 0.7 \\
\hline Education Attainment & 0.5 & 0.7 \\
\hline
\end{tabular}

\section{A.2.1.1 Uncertainty values}

This factor is given a range of uncertainty values from 0.1 to 0.2 because of the perceived ability of the existing indicators to fully address the themes of the factor. The addition of more educational-focused indicators could further reduce the uncertainty.

Factor Uncertainty Min: $\quad 0.1$

Factor Uncertainty Max: $\quad 0.2$

\section{A.2.1.2 Example outcome}

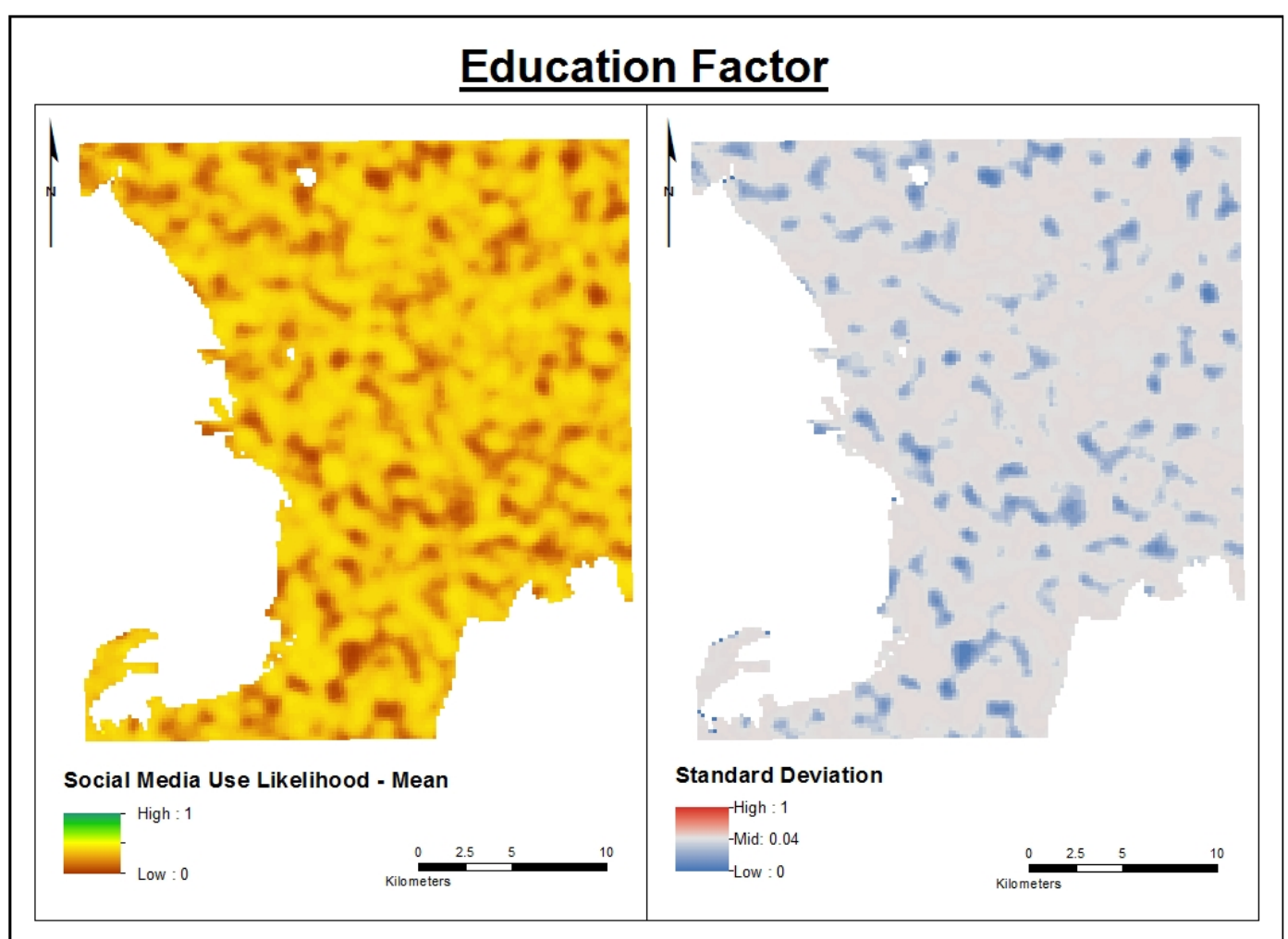




\section{A.2.1.3 References}

Albert, Jose Ramon G., Ramonette B. Serafica and Beverly T. Lumbera. Examining Trends in ICT Statistics: How Does the Philippines Fare in ICT? Philippines Institute for Development Studies. May 2016.

https://dirp3.pids.gov.ph/websitecms/CDN/PUBLICATIONS/pidsdps1616.pdf.

\section{A.2.2 Indicator: Language abilities}

The Language Abilities indicator accounts for different languages spoken by members of the sample population and represented in social media posts/tweets. As the English language is often overrepresented in study area tweets in terms of actual language skills and dialects of the population as a whole, it is currently the only metric in this indicator.

\section{A.2.2.1 Metric weighting logic}

As there is only one metric in this indicator, it is given both a minimum and maximum value of 1.0.

\begin{tabular}{|l|c|c|}
\hline Metric & Weight Minimum Value & Weight Maximum Value \\
\hline SpeaksEnglish & 1.0 & 1.0 \\
\hline
\end{tabular}

\section{A.2.2.2 Uncertainty values}

This indicator is given an uncertainty minimum value of 0.1 and an uncertainty maximum value of 0.2 because of the perceived ability of the existing metrics to fully address the themes of the indicator. Additional metrics encompassing other language skills, such as local dialects, as well as metrics that analyze what language is most represented in local tweets, could further reduce the uncertainty.

\begin{tabular}{|l|l|}
\hline Indicator Uncertainty Min: & 0.1 \\
\hline Indicator Uncertainty Max: & 0.2 \\
\hline
\end{tabular}




\section{A.2.2.3 Example outcome}

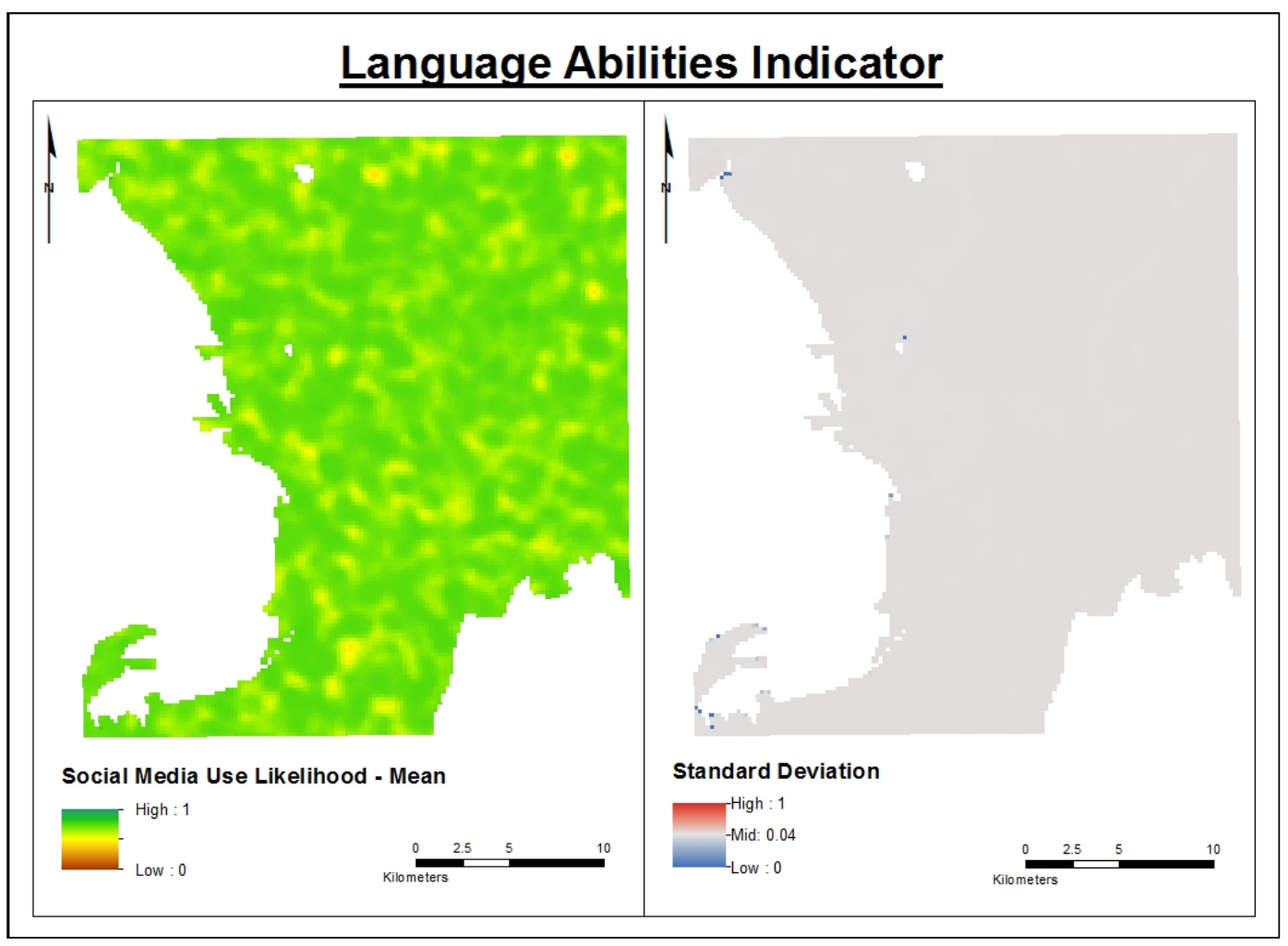

\section{A.2.2.4 Metric: SpeaksEnglish}

Indicator: Language Abilities

Factor: Education

Condition: Population

Framework: Social Media User

Metric Assigned $\quad$ Yes (min: 0.75, max: 1.0)

Values:

No (min: 0.25, max: 0.5)

Unknown (min: 0.25, max: 1.0)

Survey: $\quad$ IPUMS

Survey Date: $\quad 2000$

Other Data $\quad$ Andrei et al, 2016; Kroulek, 2017

Sources: 
Logic:

This metric concerns whether or not the survey respondent speaks English from the IPUMS survey. Answers of "Yes" are given a minimum of 0.75 (minimal risk) and a maximum value of 1.0 (minimal risk with no impact on risk, $\left.1 / 2^{0}\right)$. Answers of "No" are given a minimum value of 0.25 (medium risk, $1 / 2^{2}$ ) and a maximum value of 0.5 (slight risk, $1 / 2^{1}$ ). Answers of "Unknown" are given a minimum value of 0.25 and a maximum value of 1.0.

According to Andrei et al. (2016), 80\% of tweets from the Philippines are in English and 20\% are in Filipino. English is one of two official languages in the Philippines (the other is Filipino), and over 92\% of the population can speak it as a second language (Kroulek 2017). Maps will range from medium risk to no risk.

Example Realization Metric Map

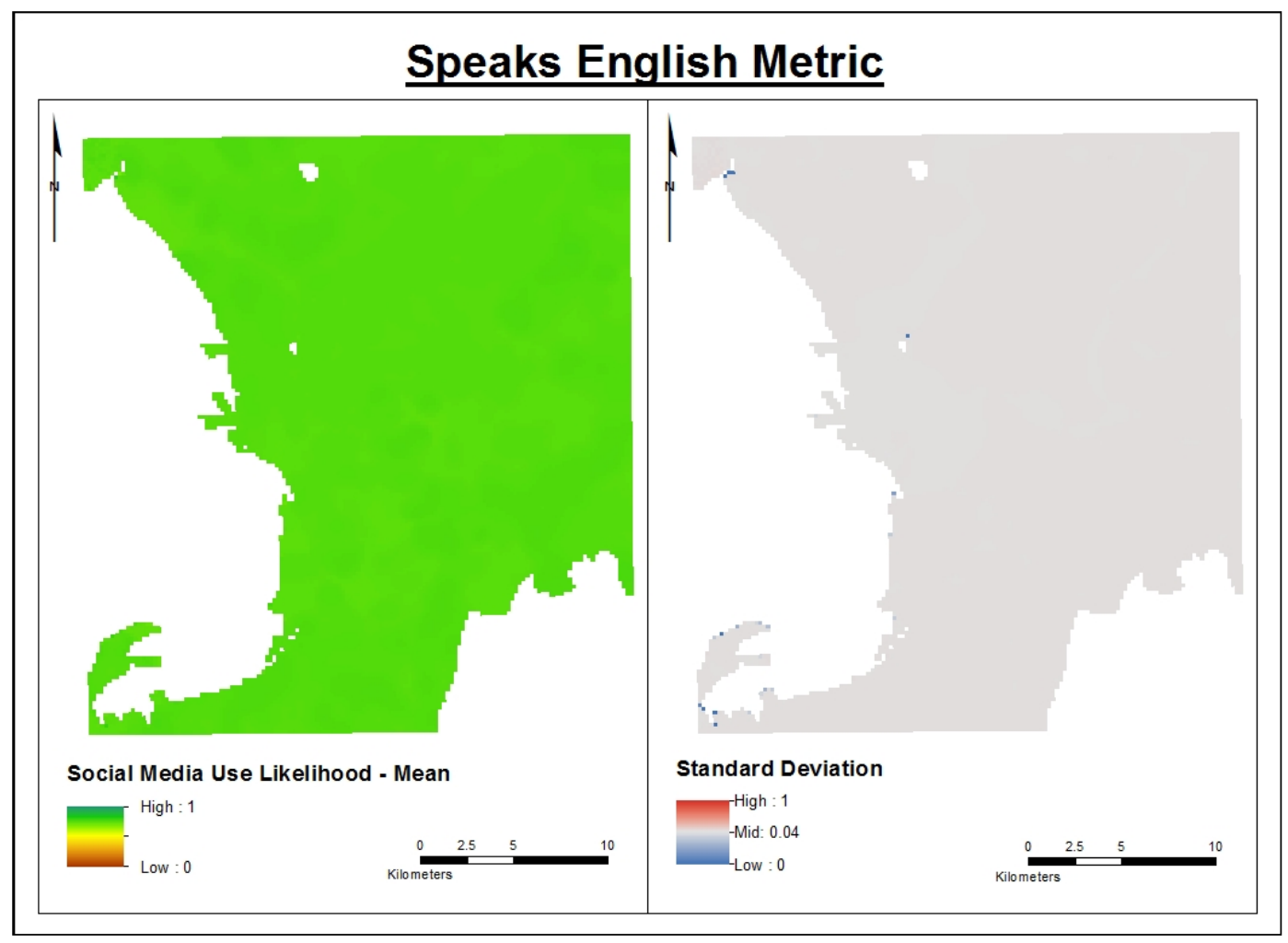

\section{A.2.2.5 References}

Andrei, Amanda, Sara Beth Elson, Guido Zarrella. Language and Emotion in Philippine Twitter Use during Typhoon Haiyan. 2016. DOI 10.13140/RG.2.1.4671.5923.

Kroulek, Alison. Which Counties Have the Most English Speakers? K International. 27 Feb 2017. http://www.k-international.com/blog/countries-with-the-most-english-speakers/.

\section{A.2.3 Indicator: Literacy}

The Literacy indicator accounts for literacy levels of individuals in the sample population. The regular use of social media, by nature require the 
skills to read and write. Literacy is identified in the ICT Development Index as an indicator on ICT capability or skills (Albert et al. 2016). There is currently only one metric within this indicator.

\section{A.2.3.1 Metric weighting logic}

As there is only one metric in this indicator, it is given both a minimum and maximum value of 1.0.

\begin{tabular}{|l|c|c|}
\hline Metric & Weight Minimum Value & Weight Maximum Value \\
\hline Literacy & 1.0 & 1.0 \\
\hline
\end{tabular}

\section{A.2.3.2 Uncertainty values}

This indicator is given an uncertainty minimum value of 0.1 and an uncertainty maximum value of 0.2 because of the perceived ability of the existing metrics to fully address the themes of the indicator. Additional metrics that represent a more detailed understanding of literacy levels (i.e., $4^{\text {th }}$ grade level, college level, etc.) could further reduce uncertainty values.

Indicator Uncertainty Min:

Indicator Uncertainty Max:
0.1

0.2 


\title{
A.2.3.3 Example outcome
}

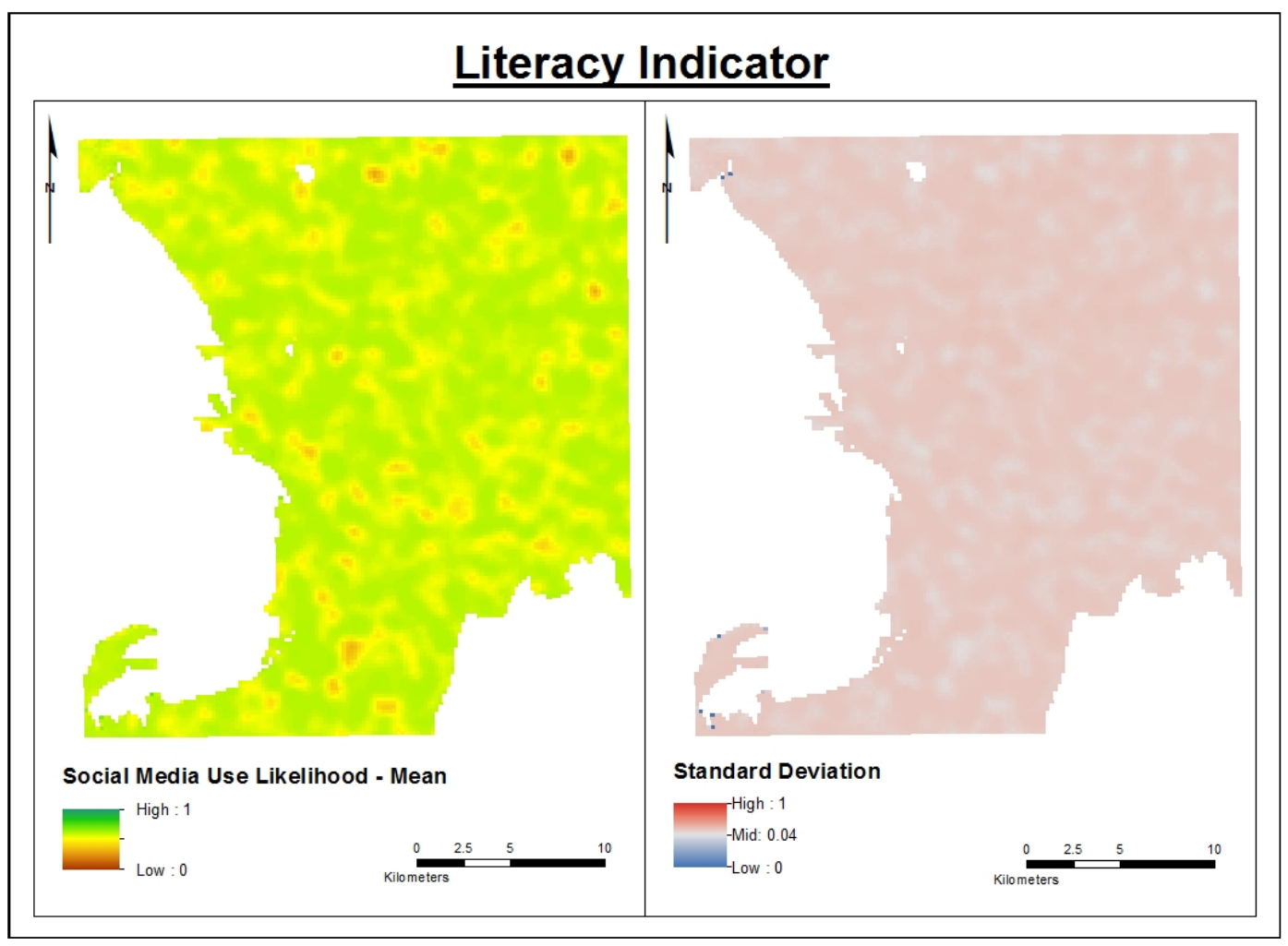

\section{A.2.3.4 References}

Albert, Jose Ramon G., Ramonette B. Serafica and Beverly T. Lumbera. Examining Trends in ICT Statistics: How Does the Philippines Fare in ICT? Philippines Institute for Development Studies. May 2016. https://dirp3.pids.gov.ph/websitecms/CDN/PUBLICATIONS/pidsdps1616.pdf.

\author{
A.2.3.5 Metric: Literacy \\ Indicator: Literacy \\ Factor: Education \\ Condition: Population \\ Framework: $\quad$ Social Media User \\ Metric Assigned $\quad$ Yes (min: 0.125, max: 1.0) \\ Values: $\quad$ No (min: 0.001, $\max : 0.0625)$ \\ Survey: IPUMS \\ Survey Date: $\quad 2000$ \\ Other Data N/A \\ Sources:
}

Logic:

This metric consists of answers to the question on whether or not the respondent is literate from the IPUMS survey. Answers of "Yes" are 
given a minimum value of 0.125 (high risk, $1 / 2^{3}$ ) and a maximum value of 1.0 (minimal risk with no impact on risk, $1 / 2^{0}$ ). Answers of "No" are given a minimum value of 0.001 (extreme risk) and a maximum value of 0.0625 (extreme risk, $1 / 2^{4}$ ). Maps will range from extreme risk to no risk.

Example Realization Metric Map

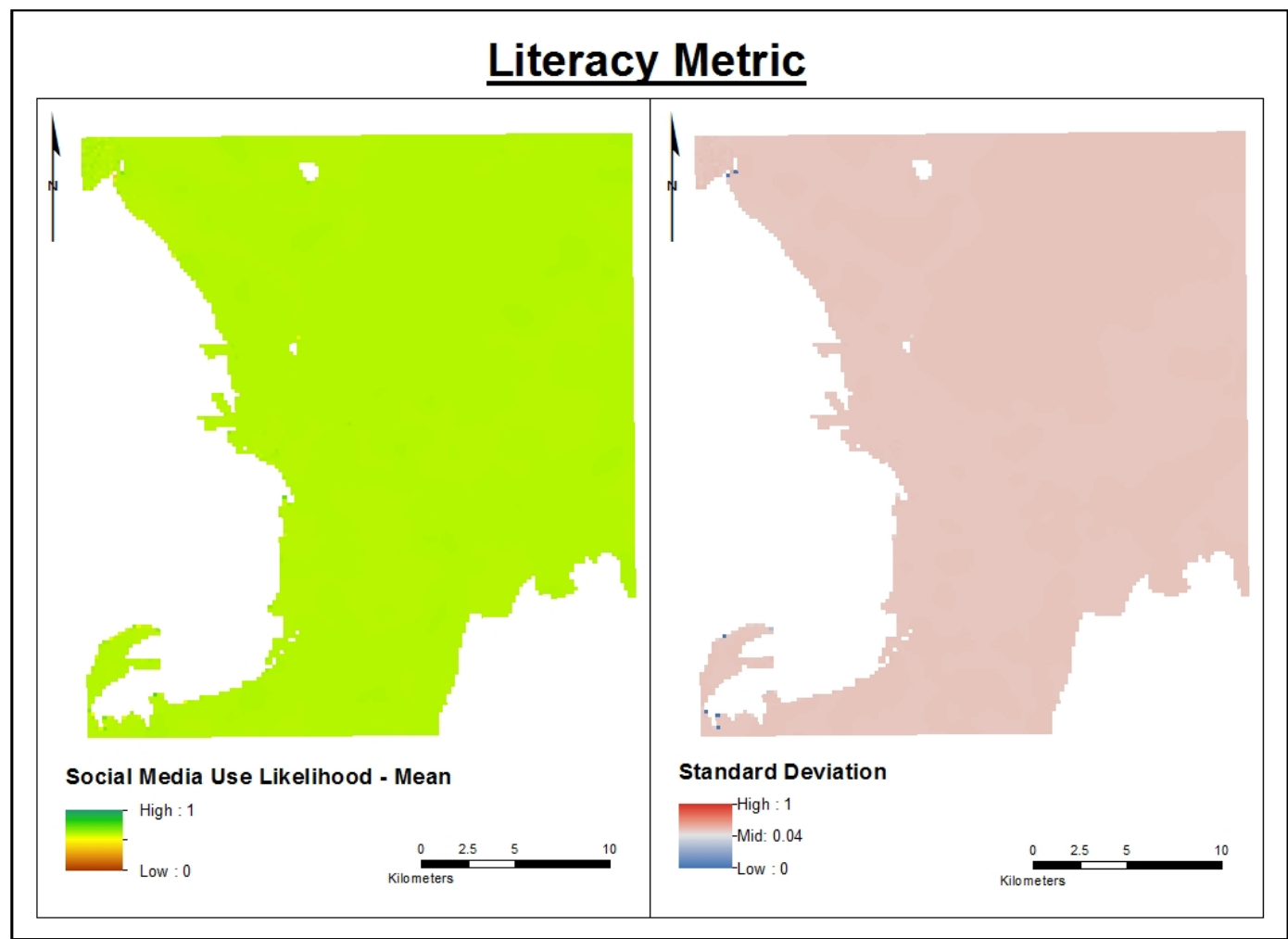

\section{A.2.4 Indicator: Educational attainment}

The Educational Attainment indicator accounts for varying educational levels of individuals in the sample population. Secondary and tertiary school enrollment is identified in the ICT Development Index as an indicator on ICT capability or skills (Albert et al. 2016).

\section{A.2.4.1 Metric weighting logic}

As the EducationAttainment and HighestGradeCompleted have more provide more specific measurements than School Attendance (which just asks if the respondent has attended school ever), they are weighted higher with both a minimum value of 0.4 and a maximum value of 0.6 .

\begin{tabular}{|l|c|c|}
\hline Metric & Weight Minimum Value & Weight Maximum Value \\
\hline EducationAttainment & 0.4 & 0.6 \\
\hline SchoolAttendance & 0.2 & 0.4 \\
\hline
\end{tabular}




\begin{tabular}{|l|c|c|}
\hline Metric & Weight Minimum Value & Weight Maximum Value \\
\hline HighestGradeCompleted & 0.4 & 0.6 \\
\hline
\end{tabular}

\section{A.2.4.2 Uncertainty values}

This indicator is given an uncertainty minimum value of 0.1 and an uncertainty maximum value of 0.2 because of the perceived ability of the existing metrics to fully address the themes of the indicator. Additional metrics measuring different educational achievement standards could further reduce the uncertainty values.

Indicator Uncertainty Min:

Indicator Uncertainty Max:
0.1

0.2

\section{A.2.4.3 Example outcome}

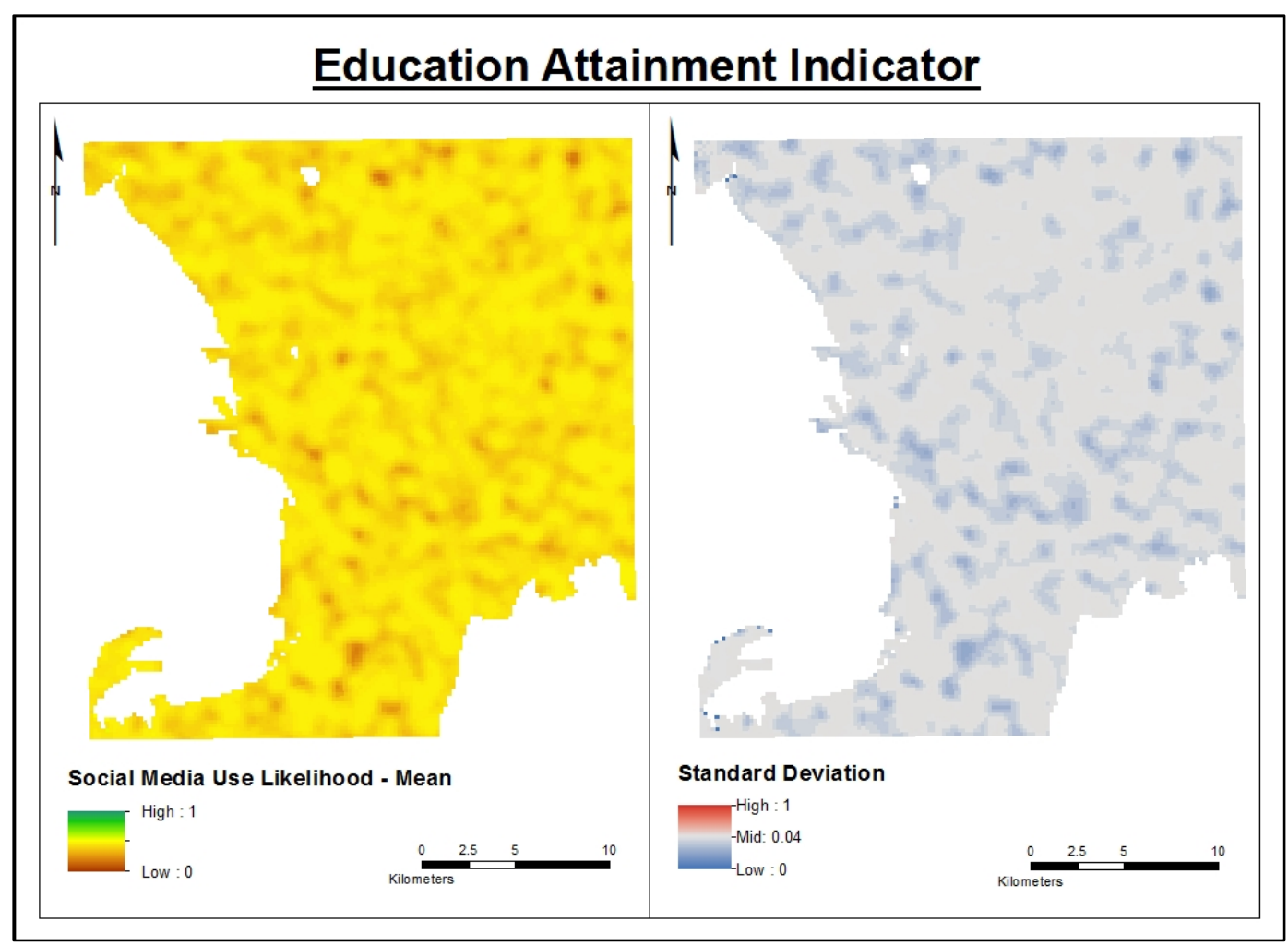

\section{A.2.4.4 References}

Albert, Jose Ramon G., Ramonette B. Serafica and Beverly T. Lumbera. Examining Trends in ICT Statistics: How Does the Philippines Fare in ICT? Philippines Institute for Development Studies. May 2016. https://dirp3.pids.gov.ph/websitecms/CDN/PUBLICATIONS/pidsdps1616.pdf. 


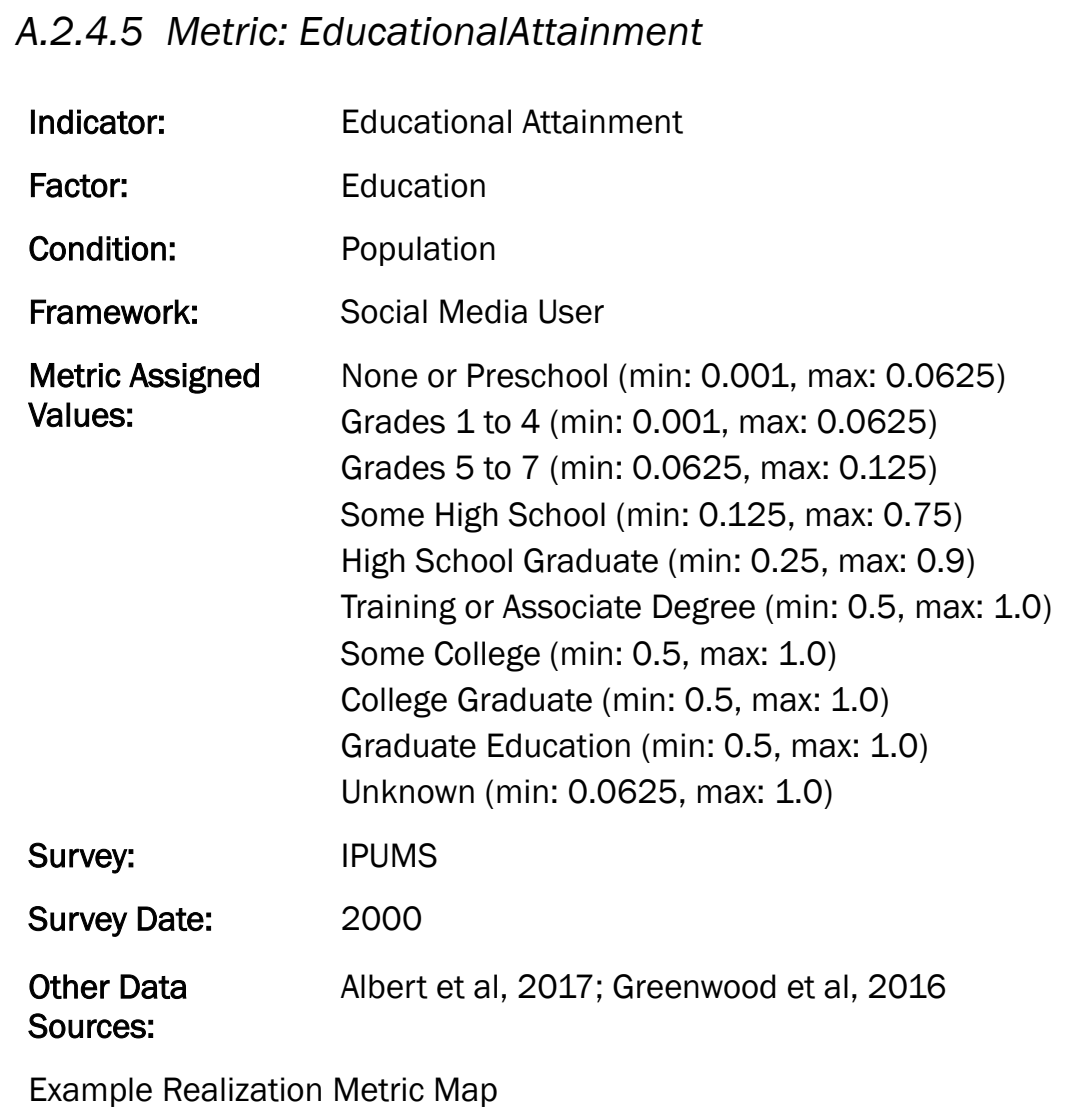

\section{Educational Attainment Metric}

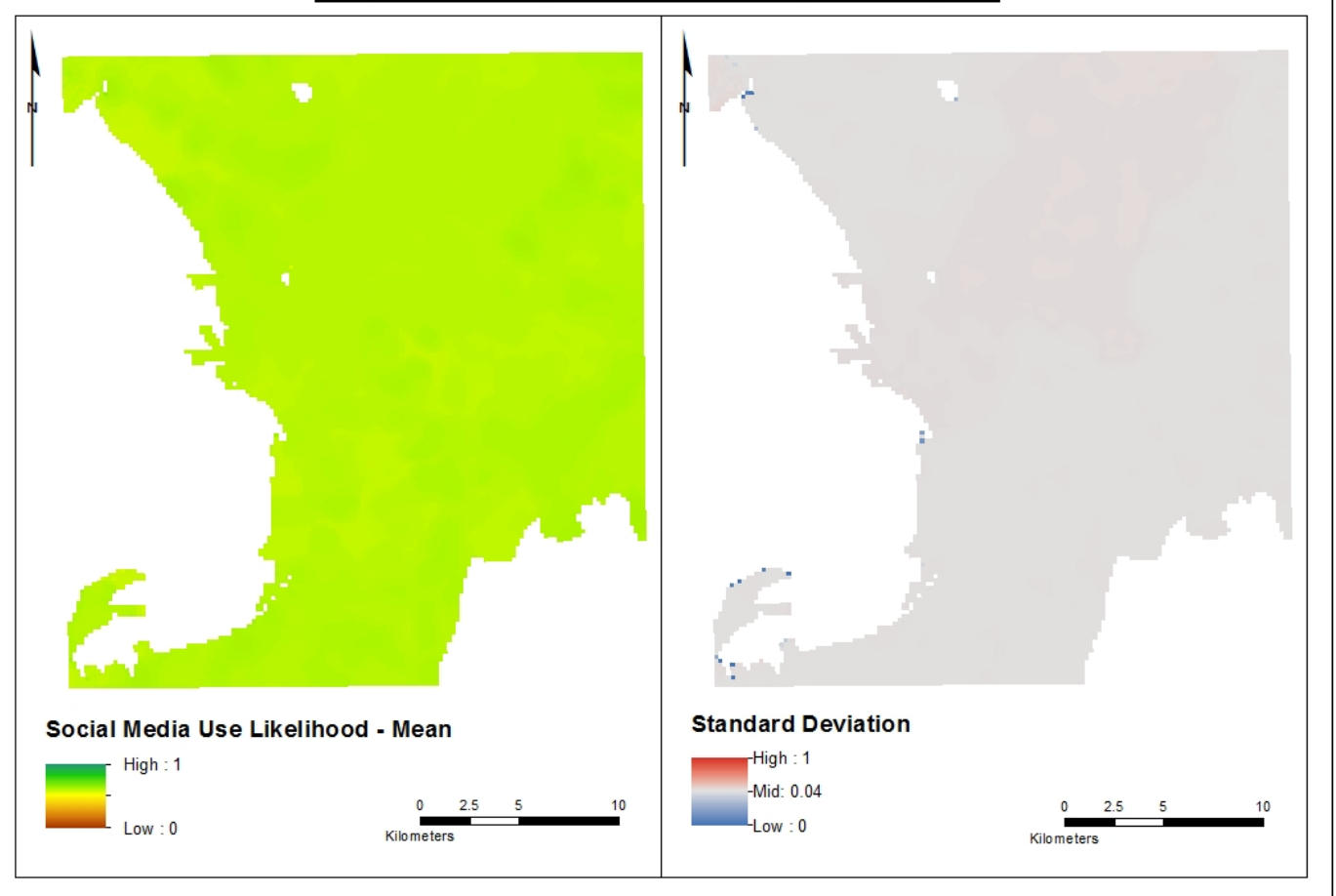




\section{A.2.4.6 References}

Albert, Jose Ramon G., Ramonette B. Serafica and Beverly T. Lumbera. Examining Trends in ICT Statistics: How Does the Philippines Fare in ICT? Philippines Institute for Development Studies. May 2016. https://dirp3.pids.gov.ph/websitecms/CDN/PUBLICATIONS/pidsdps1616.pdf.

Greenwood, Shannon, Andrew Perrin and Maeve Duggan. Social Media Update 2016. Pew Research Center. Nov 11, 2016. http://www.pewinternet.org/2016/11/11/socialmedia-update-2016/.

\section{A.2.4.7 Metric: SchoolAttendance}

\begin{tabular}{|c|c|}
\hline Indicator: & Educational Attainment \\
\hline Factor: & Education \\
\hline Condition: & Population \\
\hline Framework: & Social Media User \\
\hline $\begin{array}{l}\text { Metric Assigned } \\
\text { Values: }\end{array}$ & $\begin{array}{l}\text { Yes (min: 0.25, max: 0.9) } \\
\text { No (min: } 0.001, \max : 0.0625)\end{array}$ \\
\hline Survey: & IPUMS \\
\hline Survey Date: & 2013 \\
\hline $\begin{array}{l}\text { Other Data } \\
\text { Sources: }\end{array}$ & $\mathrm{N} / \mathrm{A}$ \\
\hline Logic: & $\begin{array}{l}\text { This metric consists of responses to the question "Ever attended } \\
\text { school?" from the DHS survey. Answers of "Yes" are given a minimum } \\
\text { value of } 0.25 \text { (medium risk, } 1 / 2^{2} \text { ) and a maximum value of } 0.9 \text { (minimal } \\
\text { risk). Answers of "No" are given a minimum value of } 0.001 \text { (extreme } \\
\text { risk) and a maximum value of } 0.0625 \text { (extreme risk, } 1 / 2^{4} \text { ). Maps will } \\
\text { range from extreme risk to minimal risk. }\end{array}$ \\
\hline
\end{tabular}

Example Realization Metric Map 


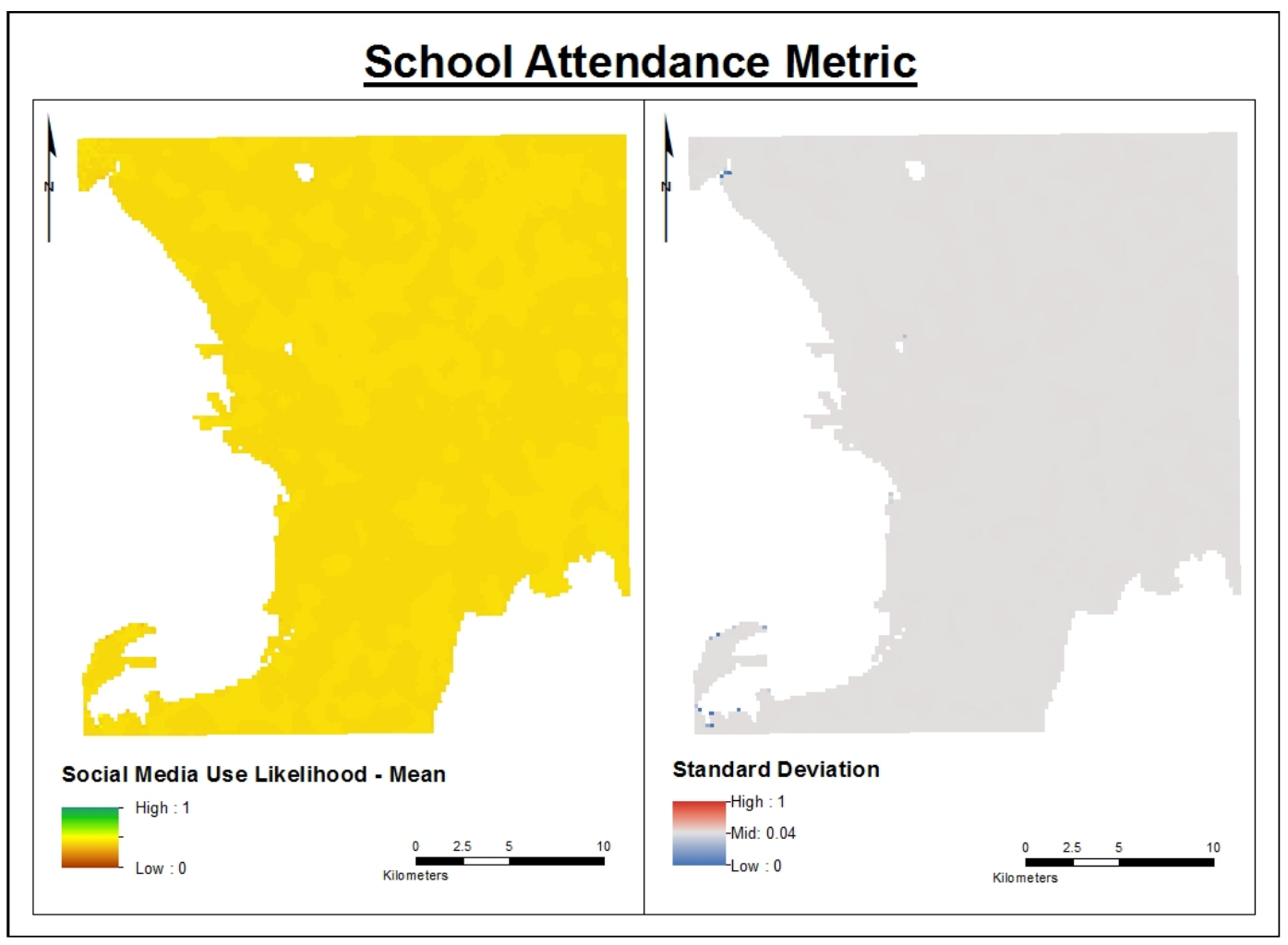

\section{A.2.4.8 Metric: HighestGradeCompleted}

Indicator: Educational Attainment

Factor:

Education

Condition:

Population

Framework:

Social Media User

Metric Assigned

NoEducation(min: 0.001, max: 0.0625)

Values:

SomeElementary (min: 0.001, max: 0.0625)

CompletedElementary (min: 0.0625, max: 0.125)

Some High School (min: 0.125, max: 0.7)

CompletedHighSchool (min: 0.5, max: 0.9)

CollegeOrHigher (min: 0.5, max: 1.0)

Survey: DHS

Survey Date: $\quad 2013$

Other Data N/A

Sources:

Logic:

This metric consists of answers to the question of highest grade completed from the women's portion of the DHS survey. Higher education levels correlate with a higher likelihood of social media use (Albert et al. 2017, Greenwood et al. 2016). Answers of "No Education" and "Some Elementary" are given a minimum value of 0.001 (extreme risk) and a maximum value of 0.0625 (extreme risk, $\left.1 / 2^{4}\right)$. Answers of "Completed Elementary" are given a minimum value of 
0.0625 and a maximum value of 0.125 (high risk, $1 / 2^{3}$ ). Answers of "Some High School" are given a minimum value of 0.125 and a maximum value of 0.7 (minimal risk). Answers of "Completed High School" are given a minimum value of 0.5 (slight risk, $1 / 2^{1}$ ) and a maximum value of 0.9 (minimal risk). Answers of "College or Higher" are given a minimum value of 0.5 and a maximum value of 1.0 (minimal risk with no impact on risk, $1 / 2^{0}$ ). Maps will range from extreme risk to no risk.

Example Realization Metric Map: Not Available

\section{A.2.4.9 References}

Greenwood, Shannon, Andrew Perrin and Maeve Duggan. Social Media Update 2016. Pew Research Center. Nov 11, 2016. http://www.pewinternet.org/2016/11/11/socialmedia-update-2016/.

\section{A.3 Technology condition overview}

This condition account for the access to/ownership of technology that enables regular social media use.

All factors currently have available metric and indicator data.

Factors within this condition include Device Access and Network Access.

\section{Factor Weighting Logic}

As one needs both a device (i.e., computer, phone, etc.) and a network connection (Ethernet, Wi-Fi, etc.) in order to use social media, both factors are weighted equally with a minimum value of 0.6 and a maximum value of 0.8 .

\begin{tabular}{|l|c|c|}
\hline Factor & Weight Minimum Value & Weight Maximum Value \\
\hline Device Access & 0.6 & 0.8 \\
\hline Network Access & 0.6 & 0.8 \\
\hline
\end{tabular}

\section{A.3.1 Uncertainty values}

This condition is given a range of uncertainty values from 0.2 to 0.4 because of the perceived ability of the existing factors to fully address the themes of the condition. The addition of factors accounting for new technological innovations, as well as the addition of qualitative data, could further reduce the uncertainty values.

Condition Uncertainty Min:

Condition Uncertainty Max:
0.2

0.4 


\section{A.3.2 Example outcome}

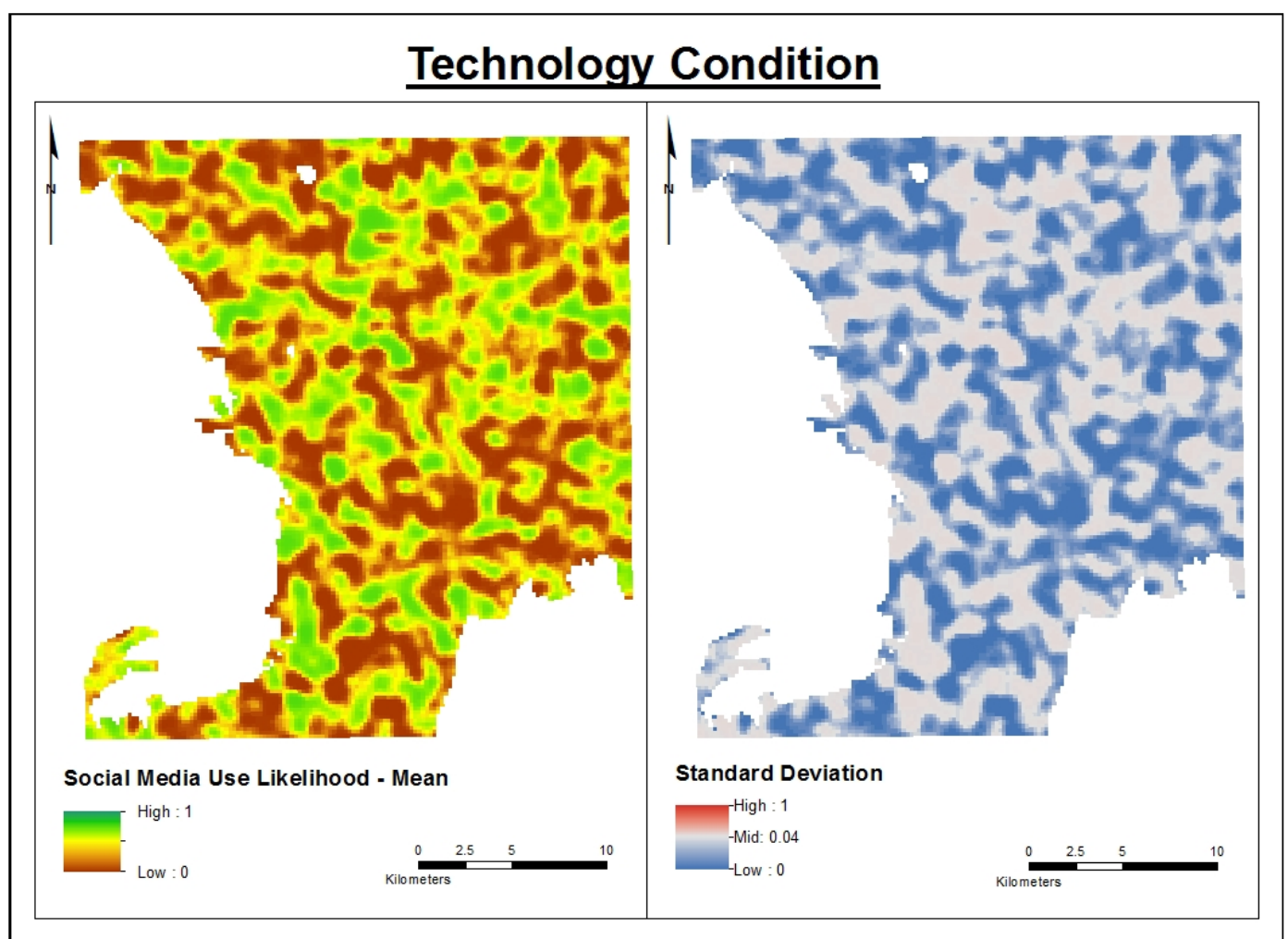

\section{A.4 Device access factor overview}

This factor accounts for whether or not members of the sample population have access to devices which enable social media use. Indicators within this factor include Phone Access and Computer Access. All indicators currently have available metric data.

\section{A.4.1 Indicator weighting logic}

As phones allow for more regular access of social media (being existentially more mobile than computers), the Phone Access indicator is given a slightly higher maximum weight value than the Computer Access indicator.

\begin{tabular}{|l|c|c|}
\hline Indicator & Weight Minimum Value & Weight Maximum Value \\
\hline Phone Access & 0.5 & 0.8 \\
\hline Computer Access & 0.5 & 0.7 \\
\hline
\end{tabular}




\section{A.4.1.1 Uncertainty values}

This factor is given a range of uncertainty values from 0.1 to 0.3 because of the perceived ability of the existing indicators to fully address the themes of the factor. The addition of indicators accounting for other devices with which one could access social media, as well as more qualitative data on the condition of the devices, could further reduce the uncertainty values.

Factor Uncertainty Min:

Factor Uncertainty Max:

\section{3}

\section{A.4.1.2 Example outcome}

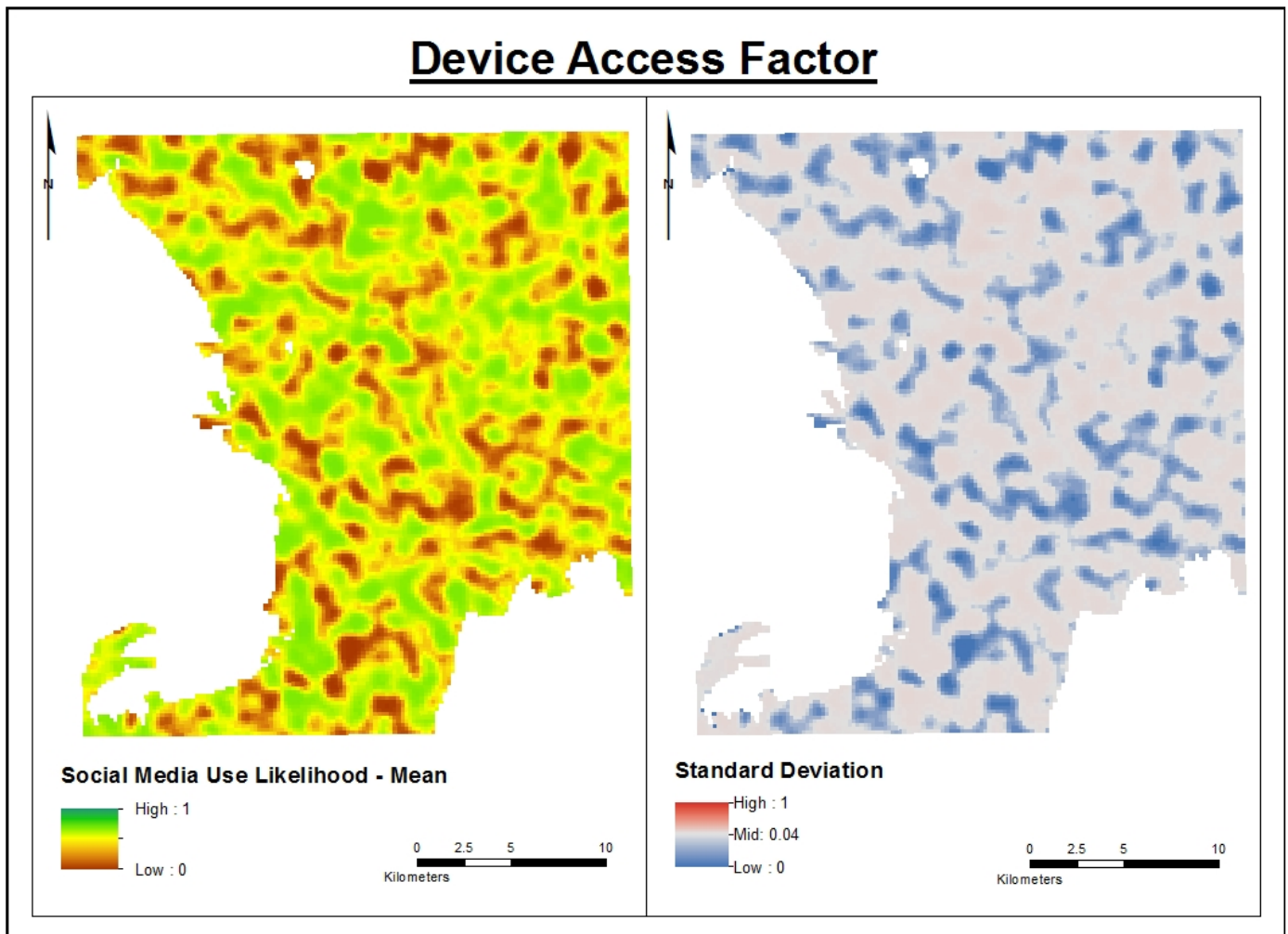

\section{A.4.2 Indicator: Phone access}

The Phone Access indicator accounts for ownership of and/or access to phones. Within the ICT Development Index, indicators on ICT infrastructure and access include: fixed telephone subscriptions, mobile cellular telephone subscriptions, international internet bandwidth per internet user, percentage of households with a computer, and percentage of households with internet access (Albert et al. 2016). 


\section{A.4.2.1 Metric weighting logic}

As cell phones are, by their nature, much more likely to have the ability to access social media (i.e., via a smartphone), the CellPhoneOwnership metric is weighted the highest. PhoneOwnership and TelephoneAvailability are weighted the same, as they essentially provide the same information but from different survey resources.

\begin{tabular}{|l|c|c|}
\hline Metric & Weight Minimum Value & Weight Maximum Value \\
\hline PhoneOwnership & 0.5 & 0.5 \\
\hline CellPhoneOwnership & 0.7 & 0.9 \\
\hline TelephoneAvailability & 0.5 & 0.5 \\
\hline
\end{tabular}

\section{A.4.2.2 Uncertainty values}

This indicator is given an uncertainty minimum value of 0.1 and an uncertainty maximum value of 0.2 because of the perceived ability of the existing metrics to fully address the themes of the indicator. The addition of more qualitative data, as well as more specific metrics such as time spent on phone, could further decrease the uncertainty values.

Indicator Uncertainty Min:

Indicator Uncertainty Max:
0.1

0.2 


\section{A.4.2.3 Example outcome}

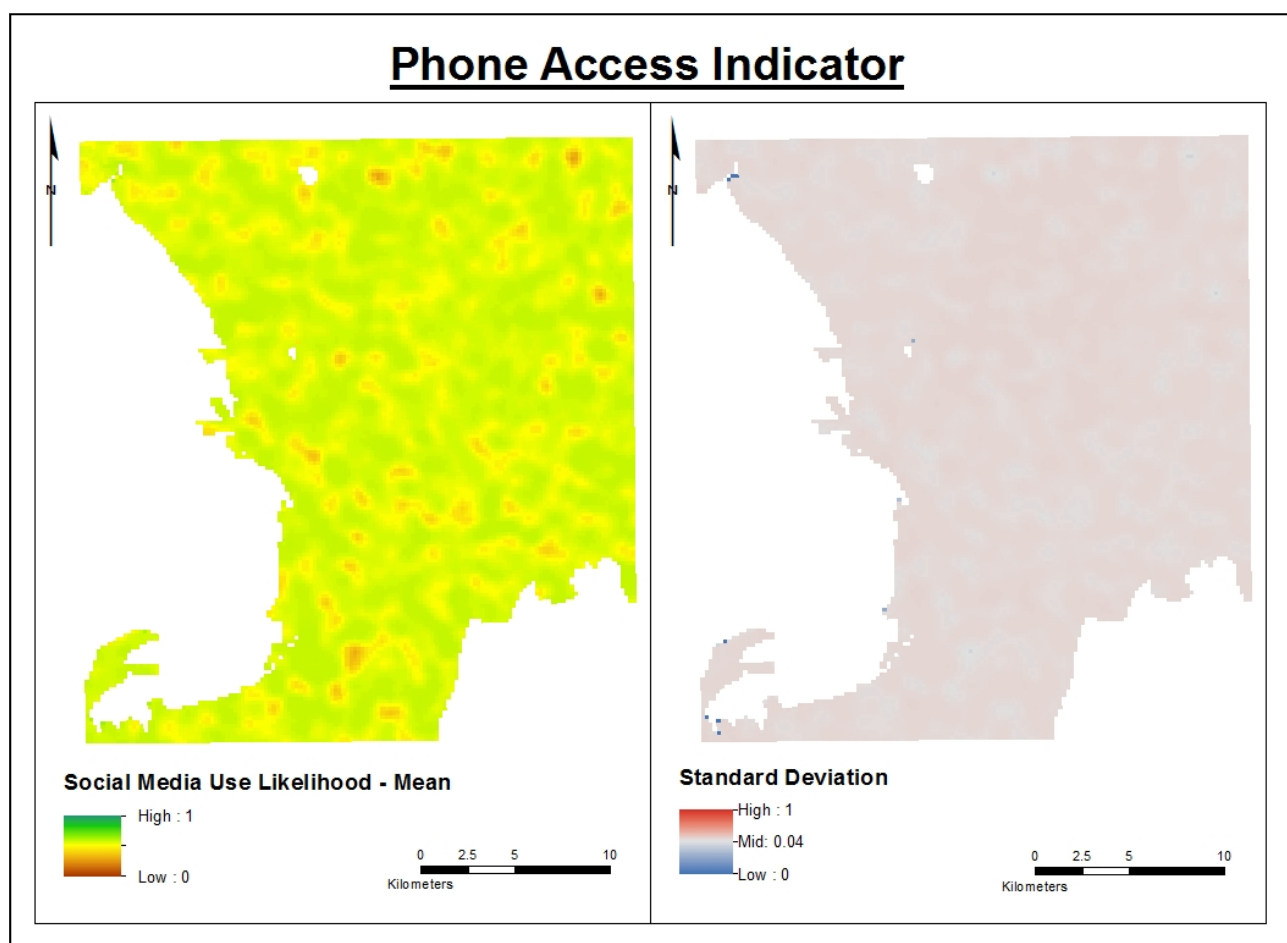

\section{A.4.2.4 References}

Albert, Jose Ramon G., Ramonette B. Serafica and Beverly T. Lumbera. Examining Trends in ICT Statistics: How Does the Philippines Fare in ICT? Philippines Institute for Development Studies. May 2016. https://dirp3.pids.gov.ph/websitecms/CDN/PUBLICATIONS/pidsdps1616.pdf.

\section{A.4.2.5 Metric: PhoneOwnership}

Indicator: Phone Access

Factor: Device Access

Condition: Technology

Framework: $\quad$ Social Media User

Metric Assigned $\quad$ Yes (min: 0.75, max: 1.0)

Values: $\quad$ No (min: 0.0625 , max: 0.5$)$

Survey: DHS

Survey Date: $\quad 2013$

Other Data N/A

Sources:

Logic:

This metric consists of answers to the question whether the household owns a landline/wireless telephone from the DHS survey. Answers of "Yes" are given a minimum value of 0.75 (minimal risk) and a maximum value of 1.0 (minimal risk with no impact on risk, $1 / 2^{0}$ ). 
Answers of "No" are given a minimum value of 0.0625 (extreme risk, $1 / 2^{4}$ ) and a maximum value of 0.5 (slight risk, $1 / 2^{1}$ ). Maps will range from extreme risk to no risk.

Example Realization Metric Map Not Available

\begin{abstract}
A.4.2.6 Metric: CellPhoneOwnership
Indicator: Phone Access

Factor: Device Access

Condition: Technology

Framework: $\quad$ Social Media User

Metric Assigned Yes (min: 0.75, max: 1.0)

Values: $\quad$ No (min: 0.0625 , max: 0.5)

Survey: $\quad$ DHS

Survey Date: $\quad 2013$

Other Data N/A

Sources:

Logic: $\quad$ This metric consists of answers to the question whether the household owns a mobile telephone from the DHS survey. Answers of "Yes" are given a minimum value of 0.75 (minimal risk) and a maximum value of 1.0 (minimal risk with no impact on risk, $1 / 2^{0}$ ). Answers of "No" are given a minimum value of 0.0625 (extreme risk, $1 / 2^{4}$ ) and a maximum value of 0.5 (slight risk, $1 / 2^{1}$ ). Maps will range from extreme risk to no risk.
\end{abstract}

Example Realization Metric Map: Not Available

$\begin{array}{ll}\text { A.4.2.7 Metric: } & \text { TelephoneAvailability } \\ \text { Indicator: } & \text { Phone Access } \\ \text { Factor: } & \text { Device Access } \\ \text { Condition: } & \text { Technology } \\ \text { Framework: } & \text { Social Media User } \\ \text { Metric Assigned } & \text { Yes (min: 0.75, max: 1.0) } \\ \text { Values: } & \text { No (min: 0.0625, max: 0.5) } \\ \text { Survey: } & \text { IPUMS } \\ \text { Survey Date: } & \text { 2000 } \\ \text { Other Data } & \text { N/A } \\ \text { Sources: } & \end{array}$

Logic:

This metric consists of answers to the question whether the household has access to a telephone from the IPUMS survey. Answers of "Yes" are given a minimum value of 0.75 (minimal risk) and a maximum value of 1.0 (minimal risk with no impact on risk, $1 / 2^{0}$ ). Answers of "No" are given a minimum value of 0.0625 (extreme risk, $1 / 2^{4}$ ) and a maximum value of 0.5 (slight risk, $1 / 2^{1}$ ). Maps will range from extreme risk to no risk. 
Example Realization Metric Map

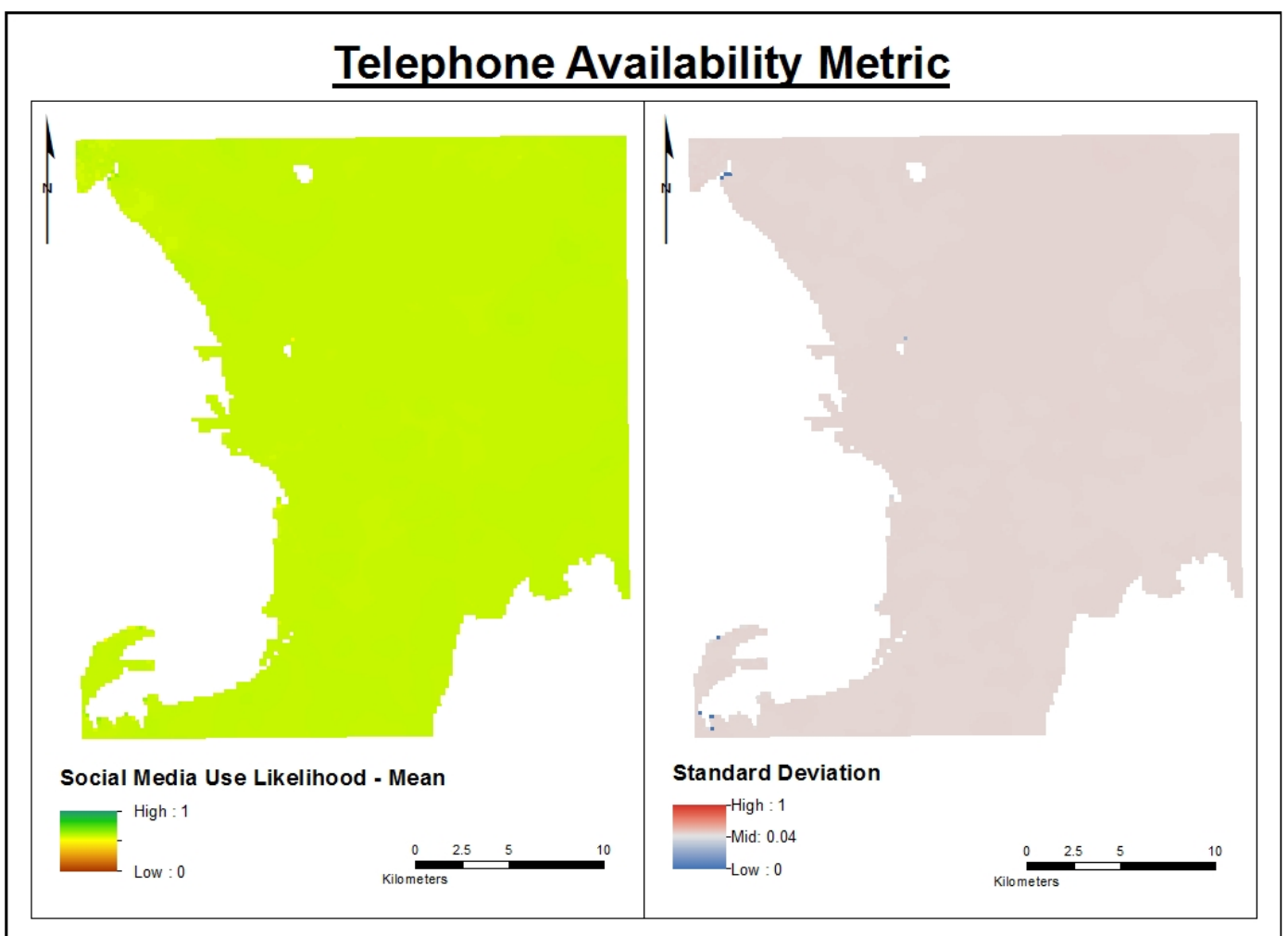

\section{A.4.3 Indicator: Computer access}

The Computer Access indicator accounts for ownership of and/or access to computers. Within the ICT Development Index, indicators on ICT infrastructure and access include: fixed telephone subscriptions, mobile cellular telephone subscriptions, international internet bandwidth per internet user, percentage of households with a computer, and percentage of households with internet access (Albert et al. 2016).

\section{A.4.3.1 Metric weighting logic}

As there is only one metric in this indicator, it is given both a minimum and maximum value of 1.0 .

\begin{tabular}{|l|c|c|}
\hline Metric & Weight Minimum Value & Weight Maximum Value \\
\hline PersonalComputerOwnership & 1.0 & 1.0 \\
\hline
\end{tabular}




\section{A.4.3.2 Uncertainty values}

This indicator is given an uncertainty minimum value of 0.1 and an uncertainty maximum value of 0.2 because of the perceived ability of the existing metrics to fully address the themes of the indicator. The addition of more qualitative data, as well as more specific metrics such as time spent on computers/tablets, could further decrease the uncertainty values.

Indicator Uncertainty Min:

Indicator Uncertainty Max:

\section{A.4.3.3 Example outcome}

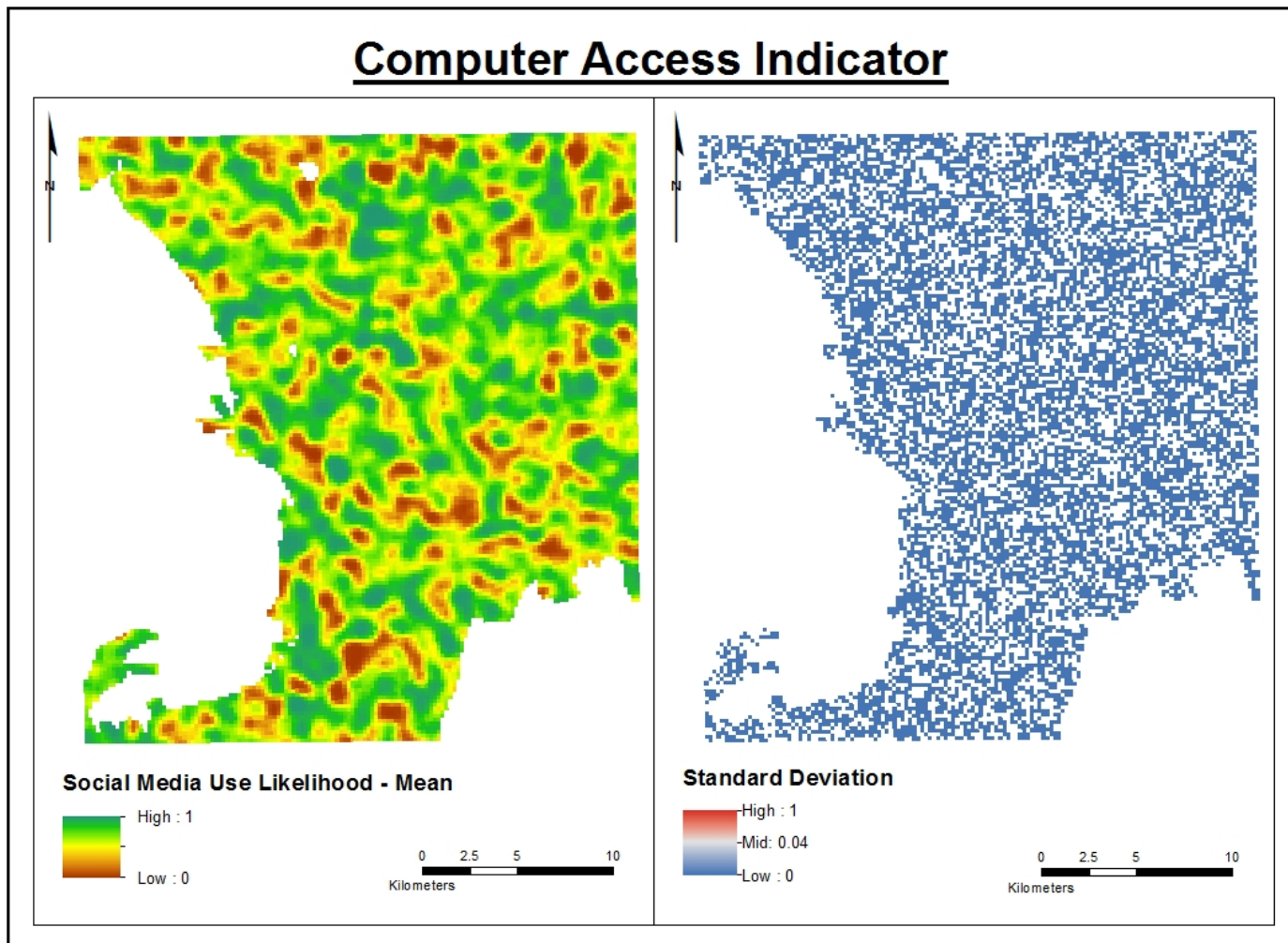

\section{Reference}

Albert, Jose Ramon G., Ramonette B. Serafica and Beverly T. Lumbera. Examining Trends in ICT Statistics: How Does the Philippines Fare in ICT? Philippines Institute for Development Studies. May 2016.

https://dirp3.pids.gov.ph/websitecms/CDN/PUBLICATIONS/pidsdps1616.pdf.

\section{A.4.3.4 Metric: PersonalComputerOwnership}

Indicator: Computer Access

Factor: Device Access 


\begin{tabular}{|c|c|}
\hline Condition: & Technology \\
\hline Framework: & Social Media User \\
\hline Metric Assigned & Yes (min: 0.75, max: 1.0) \\
\hline Values: & No (min: 0.0625, max: 0.5) \\
\hline Survey: & DHS \\
\hline Survey Date: & 2013 \\
\hline $\begin{array}{l}\text { Other Data } \\
\text { Sources: }\end{array}$ & $\mathrm{N} / \mathrm{A}$ \\
\hline Logic: & $\begin{array}{l}\text { This metric consists of answers to the question whether the household } \\
\text { owns a personal computer or laptop from the DHS survey. Answers of } \\
\text { "Yes" are given a minimum value of } 0.75 \text { (minimal risk) and a } \\
\text { maximum value of } 1.0 \text { (minimal risk with no impact on risk, } 1 / 2^{0} \text { ). } \\
\text { Answers of "No" are given a minimum value of } 0.0625 \text { (extreme risk, } \\
1 / 2^{4} \text { ) and a maximum value of } 0.5 \text { (slight risk, } 1 / 2^{1} \text { ). Maps will range from } \\
\text { extreme risk to no risk. }\end{array}$ \\
\hline
\end{tabular}

Example Realization Metric Map: Not Available

\section{A.5 Network access factor overview}

This factor accounts for whether or not members of the sample population have access to the internet, which enable social media use. Indicators within this factor include Internet Access. All indicators currently have available metric data.

\section{A.5.1 Indicator weighting logic}

As there is only one indicator in this factor, it is given both a minimum and maximum value of 1.0.

\begin{tabular}{|l|c|c|}
\hline Indicator & Weight Minimum Value & Weight Maximum Value \\
\hline Internet Access & 1.0 & 1.0 \\
\hline
\end{tabular}

\section{A.5.1.1 Uncertainty values}

This factor is given a range of uncertainty values from 0.4 to 0.6 because of the perceived ability of the existing indicators to partially address the themes of the factor. The addition of indicators measuring cell phone network coverage, cell phone tower locations, available Wi-Fi locations, etc., could further decrease the uncertainty values.

Factor Uncertainty Min:

Factor Uncertainty Max:
0.4

0.6 


\section{A.5.1.2 Example outcome}

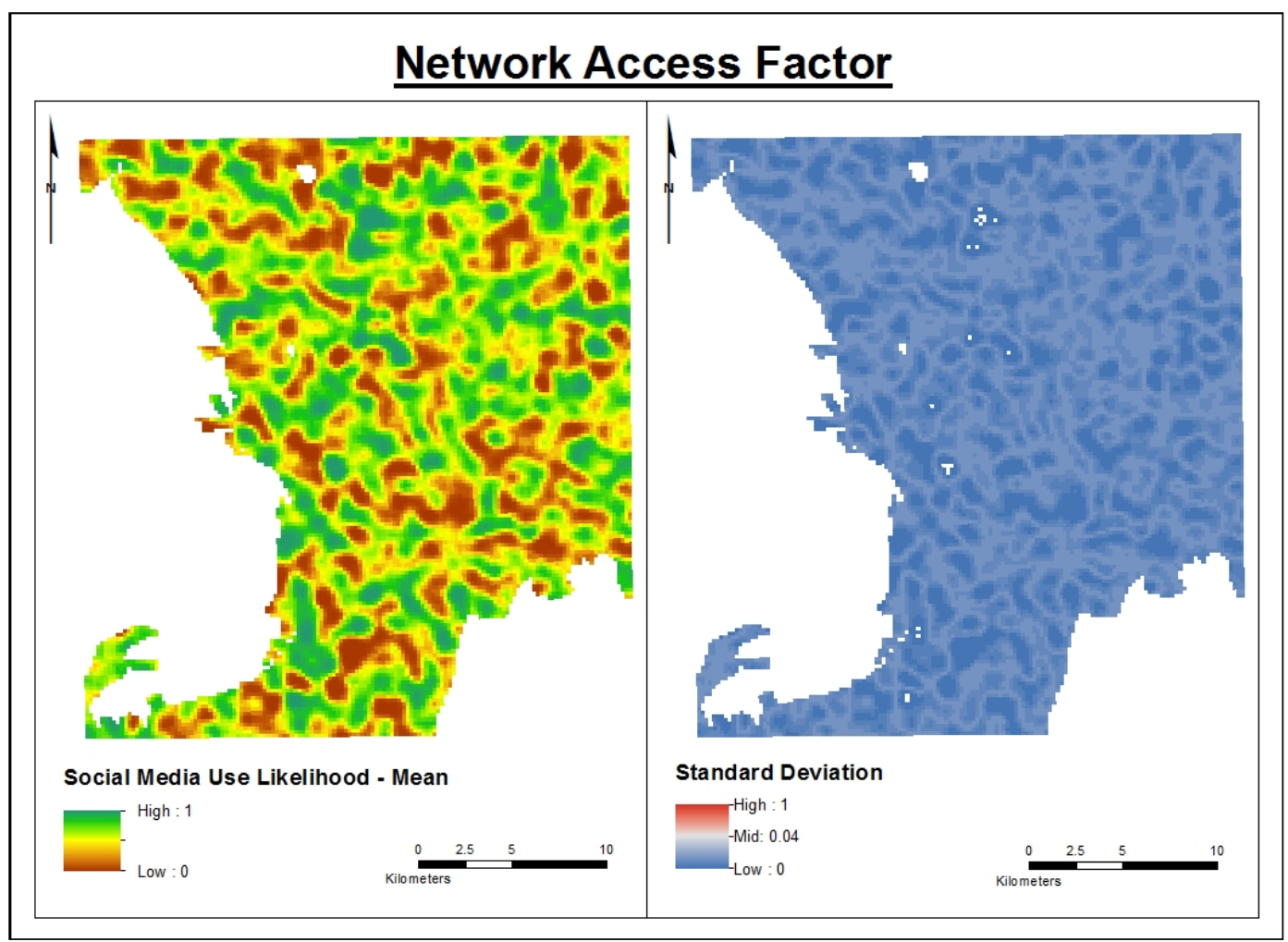

\section{A.5.2 Indicator: internet access}

The Internet Access indicator accounts for household access to the internet. Within the ICT Development Index, indicators on ICT infrastructure and access include: fixed telephone subscriptions, mobile cellular telephone subscriptions, international internet bandwidth per internet user, percentage of households with a computer, and percentage of households with internet access (Albert et al. 2016). Also, within the ICT Development Index, indicators on ICT intensity and usage include: individuals using the internet, fixed broadband subscriptions, and wireless broadband subscriptions (Albert et al. 2016).

\section{A.5.2.1 Metric weighting logic}

As there is only one metric in this indicator, it is given both a minimum and maximum value of 1.0.

\begin{tabular}{|l|c|c|}
\hline Metric & Weight Minimum Value & Weight Maximum Value \\
\hline EmaillnternetCheck & 1.0 & 1.0 \\
\hline
\end{tabular}




\section{A.5.2.2 Uncertainty values}

This indicator is given an uncertainty minimum value of 0.1 and an uncertainty maximum value of 0.3 The addition of more qualitative data, as well as more specific metrics such as time spent online (and especially time spent on Twitter/other social media websites), could further decrease the uncertainty values.

Indicator Uncertainty Min:

Indicator Uncertainty Max:
0.1

0.3

\section{A.5.2.3 Example outcome}

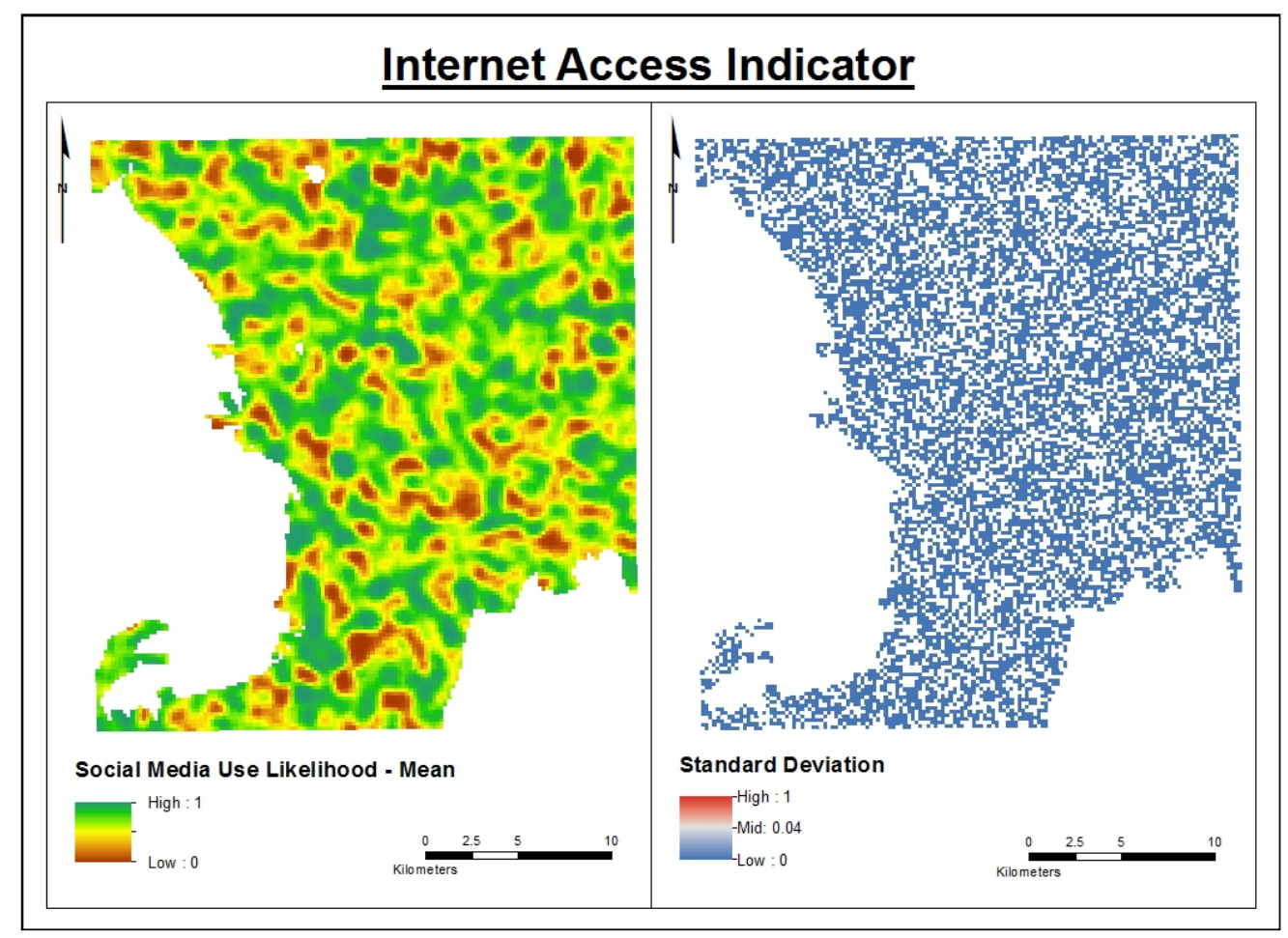

\section{A.5.2.4 References}

Albert, Jose Ramon G., Ramonette B. Serafica and Beverly T. Lumbera. Examining Trends in ICT Statistics: How Does the Philippines Fare in ICT? Philippines Institute for Development Studies. May 2016.

https://dirp3.pids.gov.ph/websitecms/CDN/PUBLICATIONS/pidsdps1616.pdf.

\section{A.5.2.5 Metric: EmaillnternetCheck}

$\begin{array}{ll}\text { Indicator: } & \text { Internet Access } \\ \text { Factor: } & \text { Network Access } \\ \text { Condition: } & \text { Technology }\end{array}$




\begin{tabular}{|c|c|}
\hline Framework: & Social Media User \\
\hline \multirow{3}{*}{$\begin{array}{l}\text { Metric Assigned } \\
\text { Values: }\end{array}$} & At least once a week (min: $0.75, \max : 1.0)$ \\
\hline & Less than once a week (min: $0.5, \max : 0.75)$ \\
\hline & Not at all (min: 0.0625, max: 0.25) \\
\hline Survey: & $\mathrm{DHS}$ \\
\hline Survey Date: & 2013 \\
\hline $\begin{array}{l}\text { Other Data } \\
\text { Sources: }\end{array}$ & $\mathrm{N} / \mathrm{A}$ \\
\hline Logic: & $\begin{array}{l}\text { This metric consists of answers to the question of how often the } \\
\text { respondent checks email or surfs the internet from the DHS survey. } \\
\text { Answers of "At least once a week" are given a minimum value of } 0.75 \\
\text { (minimal risk) and a maximum value of } 1.0 \text { (minimal risk with no } \\
\text { impact on risk, } 1 / 2^{0} \text { ). Answers of "Less than once a week" are given a } \\
\text { minimum value of } 0.5 \text { (slight risk, } 1 / 2^{1} \text { ) and a maximum value of } 0.75 \text {. } \\
\text { Answers of "Not at all" are given a minimum value of } 0.0625 \text { (extreme } \\
\text { risk, } 1 / 2^{4} \text { ) and a maximum value of } 0.25 \text { (medium risk, } 1 / 2^{2} \text { ). Maps will } \\
\text { range from extreme risk to no risk. }\end{array}$ \\
\hline
\end{tabular}

Example Realization Metric Map

Not Available

\section{A.4 Place condition overview}

This condition accounts for the characteristics of the home (or work) environment of individuals in the sample population that could contribute to regular social media use. Legara (2015) found that tweets in the Philippines correlated with population density, Gross Regional Domestic Product (GRDP), electricity, and road networks.

All factors currently have available metric and indicator data.

Factors within this condition include Household Characteristics and Urban Characteristics.

\section{A.5.3 Factor weighting logic}

Geophysical Effects \& Consequences and Climatological Effects \& Consequences are both given a minimum weight of 0.4 and maximum weight of o.6, as they are equally important in predicting the condition opposed to Ecosystem Disruptions which has less impact on predicting cholera risk was given a minimum weight of 0.2 and maximum weight of 0.4 .

\begin{tabular}{|l|c|c|}
\hline Factor & Weight Minimum Value & Weight Maximum Value \\
\hline Household Characteristics & 0.6 & 0.8 \\
\hline Urban Characteristics & 0.4 & 0.6 \\
\hline
\end{tabular}




\section{A.5.4 Uncertainty values}

This condition is given a range of uncertainty values from 0.2 to 0.4 because of the perceived ability of the existing factors to fully address the themes of the condition. The addition of factors accounting for qualitative data, could further reduce the uncertainty values.

Condition Uncertainty Min:

0.2

Condition Uncertainty Max:

0.4

\section{A.5.5 Example outcome}

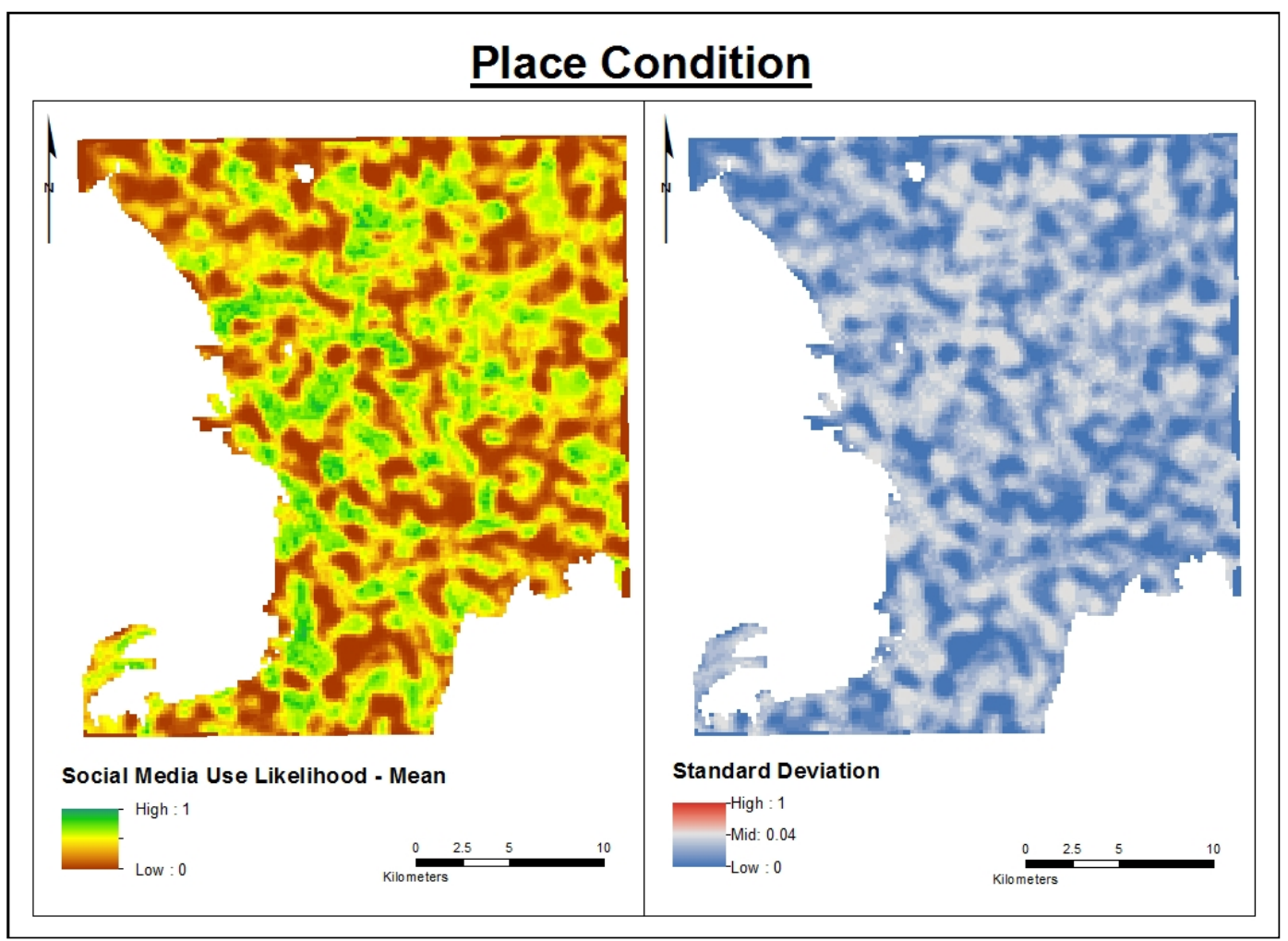

\section{A.5.6 References}

Legara E. Urbanism in the Philippines. A Byte of my 22-lb Brain. 2015. https://erikafille.ph/2015/09/10/urbanism-in-the-philippines/.

\section{A.6 Household characteristics factor overview}

This factor covers household-level characteristics that could contribute to regular social media use. Indicators within this factor include Electricity and Wealth Index. All indicators currently have available metric data. 


\section{A.6.1 Indicator weighting logic}

As the Wealth Index indicator correlates more with regular social media use than electricity, it is given both a higher minimum and maximum weight value then the Electricity indicator.

\begin{tabular}{|l|c|c|}
\hline Indicator & Weight Minimum Value & Weight Maximum Value \\
\hline Electricity & 0.4 & 0.6 \\
\hline Wealth Index & 0.7 & 0.9 \\
\hline
\end{tabular}

\section{A.6.1.1 Uncertainty values}

This factor is given a range of uncertainty values from 0.2 to 0.4 because of the perceived ability of the existing indicators to fully address the themes of the factor. The addition of indicators accounting for other household indicators, such as number of people per room, zoning, etc., could further reduce the uncertainty values.

Factor Uncertainty Min:

Factor Uncertainty Max:
0.2

0.4

\section{A.6.1.2 Example outcome}

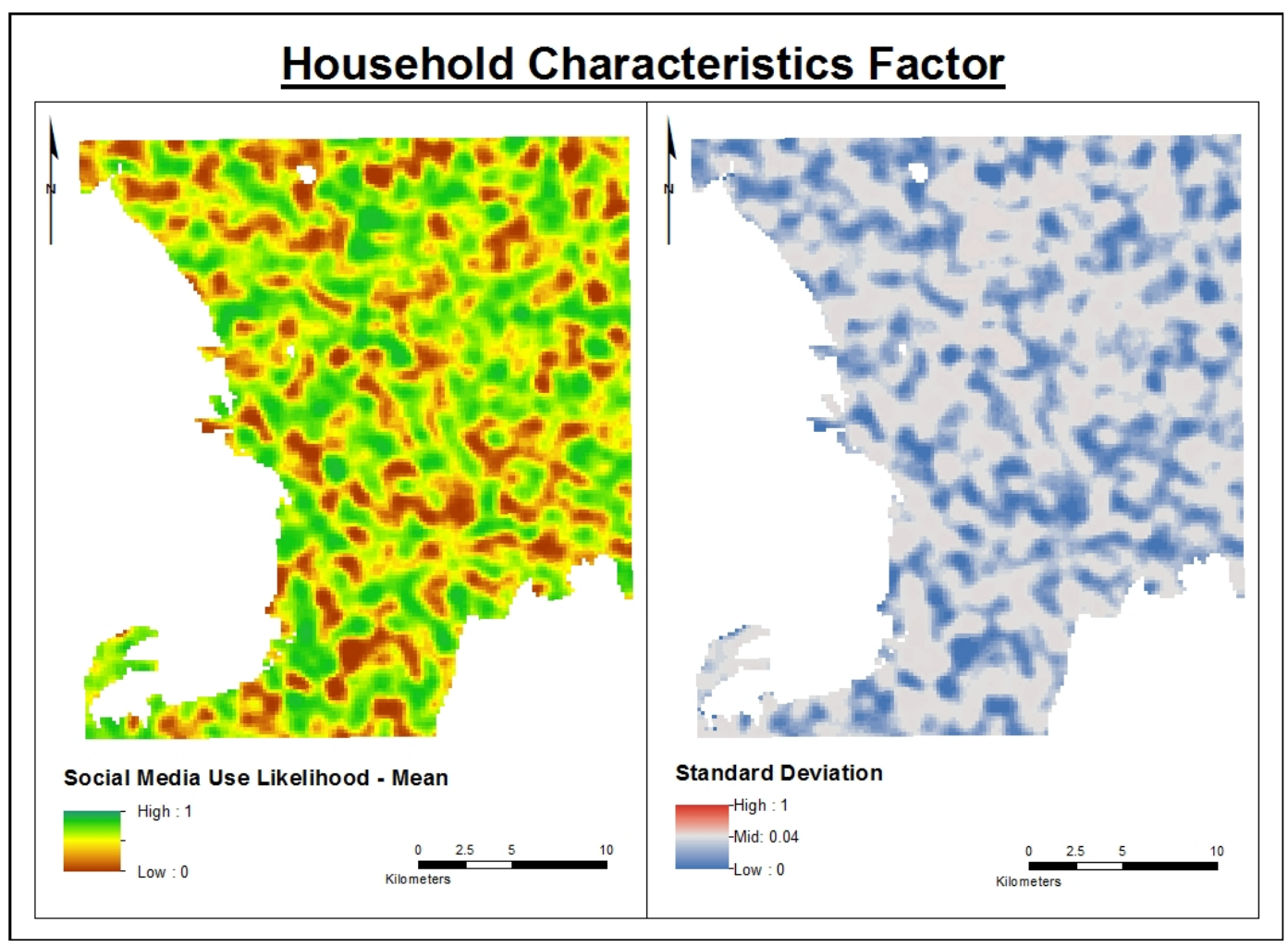




\section{A.6.2 Indicator: electricity}

The Electricity indicator accounts for whether or not a household has electricity. Legara found that tweets in the Philippines correlated with population density, GRDP, electricity, and road networks (Legara 2015).

\section{A.6.2.1 Metric weighting logic}

As both metrics within this indicator represent the same kind of data, they are weighted the same and given both a minimum and maximum value of 0.5.

\begin{tabular}{|l|c|c|}
\hline Metric & Weight Minimum Value & Weight Maximum Value \\
\hline ElectricityDHS & 0.5 & 0.5 \\
\hline ElectricityIPUMS & 0.5 & 0.5 \\
\hline
\end{tabular}

\section{A.6.2.2 Uncertainty values}

This indicator is given an uncertainty minimum value of 0.1 and an uncertainty maximum value of 0.2 because of the perceived ability of the existing metrics to fully address the themes of the indicator. The addition of qualitative data accounting for quality of electrical services could further reduce the uncertainty values.

Indicator Uncertainty Min:

Indicator Uncertainty Max:
0.1

0.2 


\section{A.6.2.3 Example outcome}

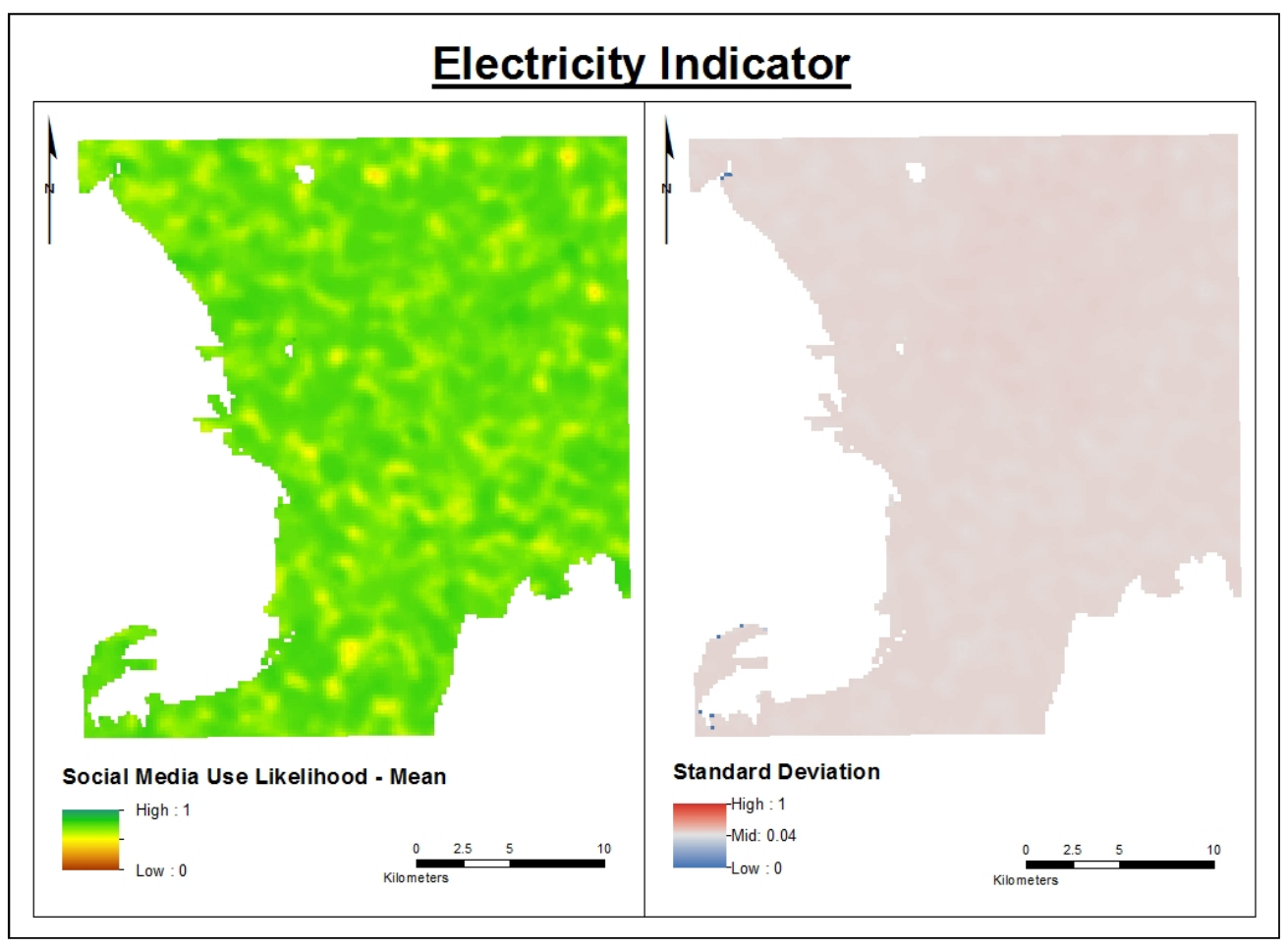

\section{A.6.2.4 References}

Legara E. Urbanism in the Philippines. A Byte of my 22-lb Brain. 2015. https://erikafille.ph/2015/09/10/urbanism-in-the-philippines/.

\section{A.6.2.5 Metric: ElectricityDHS}

$\begin{array}{ll}\text { Indicator: } & \text { Electricity } \\ \text { Factor: } & \text { Household Characteristics } \\ \text { Condition: } & \text { Place } \\ \text { Framework: } & \text { Social Media User } \\ \text { Metric Assigned } & \text { Yes (min: 0.5, max: 1.0) } \\ \text { Values: } & \text { No (min: 0.0625, max: 0.5) } \\ \text { Survey: } & \text { DHS } \\ \text { Survey Date: } & 2013 \\ \text { Other Data } & \text { N/A } \\ \text { Sources: } & \end{array}$

Logic:

This metric consists of answers to the question "Has electricity?" from the household portion of the DHS survey. Answers of "Yes" are given a minimum value of 0.5 (slight risk, $1 / 2^{1}$ ) and a maximum value of 1.0 (minimal risk with no impact on risk, $1 / 20$ ). Answers of "No" are given a minimum value of 0.0625 (extreme risk, $1 / 2^{4}$ ) and a maximum value of 0.5. Maps will range from extreme risk to no risk. 


\section{A.6.2.6 Metric: ElectricityIPUMS}

Indicator: Electricity

Factor:

Household Characteristics

Condition:

Place

Framework:

Social Media User

Metric Assigned

Yes (min: 0.5, max: 1.0)

Values:

No (min: 0.0625, max: 0.5)

Survey:

IPUMS

Survey Date:

2000

Other Data

$\mathrm{N} / \mathrm{A}$

Sources:

Logic:

This metric consists of answers to the question "Has electricity?" from the IPUMS survey. This metric consists of answers to the question "Has electricity?" from the household portion of the DHS survey. Answers of "Yes" are given a minimum value of 0.5 (slight risk, $1 / 2^{1}$ ) and a maximum value of 1.0 (minimal risk with no impact on risk, $1 / 2^{0}$ ). Answers of "No" are given a minimum value of 0.0625 (extreme risk, $1 / 2^{4}$ ) and a maximum value of 0.5 . Maps will range from extreme risk to no risk.

Example Realization Metric Map

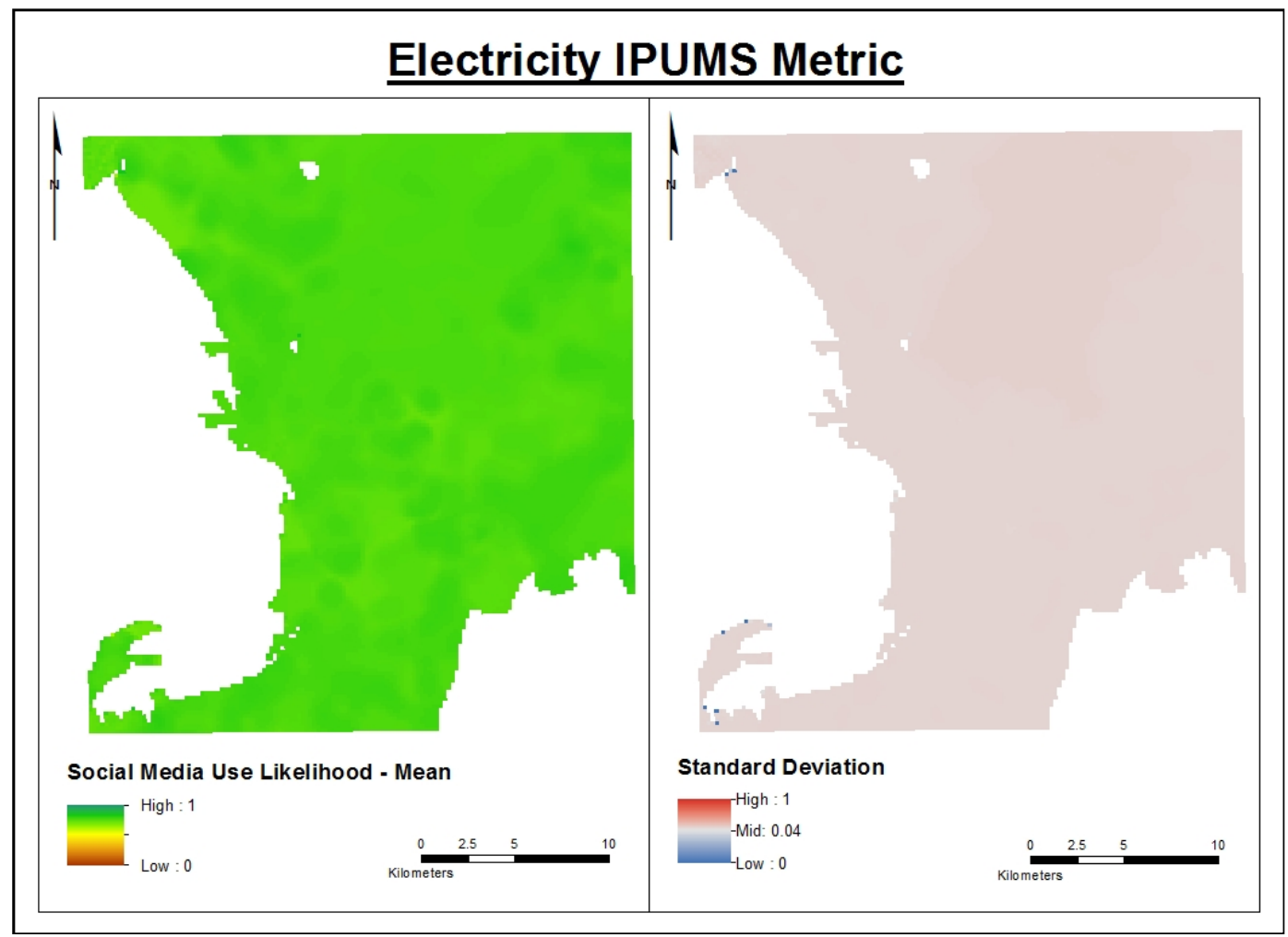




\section{A.6.3 Indicator: Wealth index}

The Wealth Index indicator accounts for socioeconomic status of the sample population. Legara found that tweets in the Philippines correlated with population density, GRDP, electricity, and road networks (Legara 2015). Wealth Index here is acting as a surrogate for GRDP.

\section{A.6.3.1 Metric weighting logic}

As there is only one metric in this indicator, it is given both a minimum and maximum value of 1.0.

\begin{tabular}{|l|c|c|}
\hline Metric & Weight Minimum Value & Weight Maximum Value \\
\hline WealthQuintiles & 1.0 & 1.0 \\
\hline
\end{tabular}

\section{A.6.3.2 Uncertainty values}

This indicator is given an uncertainty minimum value of 0.1 and an uncertainty maximum value of 0.2 because of the perceived ability of the existing metrics to fully address the themes of the indicator. The addition of data that better represents GRDP, as well as other economic indicators, could further reduce the uncertainty values.

Indicator Uncertainty Min:

Indicator Uncertainty Max:
0.1

0.2 


\section{A.6.3.3 Example outcome}

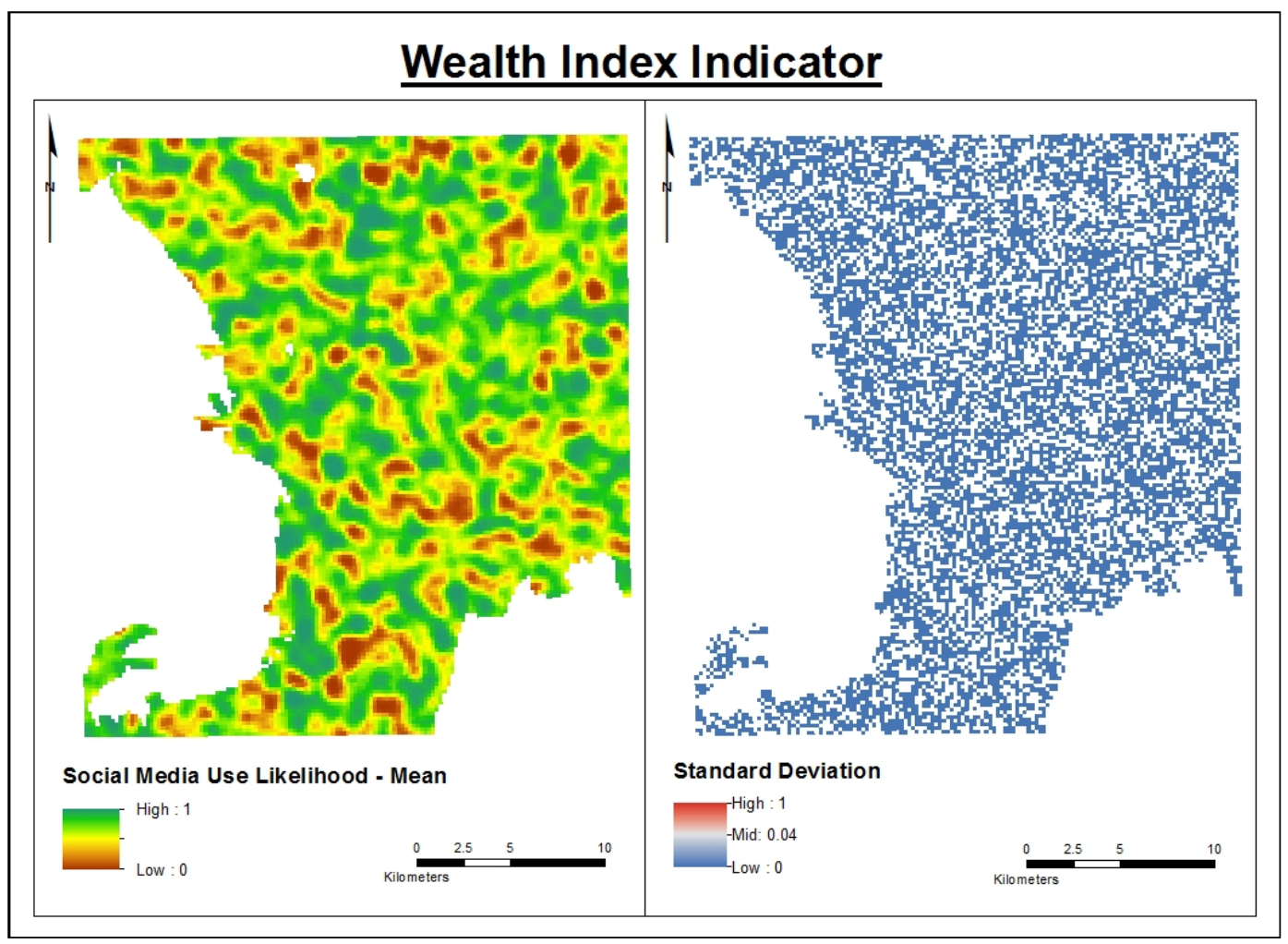

\section{A.6.3.4 References}

Legara E. Urbanism in the Philippines. A Byte of my 22-lb Brain. 2015. https://erikafille.ph/2015/09/10/urbanism-in-the-philippines/.

\section{A.6.3.5 Metric: WealthQuintiles}

$\begin{array}{ll}\text { Indicator: } & \text { Wealth Index } \\ \text { Factor: } & \text { Household Characteristics } \\ \text { Condition: } & \text { Place } \\ \text { Framework: } & \text { Social Media User } \\ \text { Metric Assigned } & \text { Lowest (min: 0.0625, max: 0.125) } \\ \text { Values: } & \text { Second (min: 0.125, max: 0.5) } \\ & \text { Middle (min: 0.25, max: 0.75) } \\ & \text { Fourth (min: 0.5, max: 1.0) } \\ & \text { Highest (min: 0.75, max: 1.0) } \\ \text { Survey: } & \text { DHS } \\ \text { Survey Date: } & 2013 \\ \text { Other Data } & \text { N/A } \\ \text { Sources: } & \end{array}$


Logic:

This metric consists of categorizations of survey respondents based on household income from the DHS survey. Answers of "Lowest" are given a minimum value of 0.0625 (extreme risk, $1 / 2^{4}$ ) and a maximum value of 0.125 (high risk, $1 / 2^{3}$ ). Answers of "Second" are given a minimum value of 0.125 and a maximum value of 0.5 (slight risk, $1 / 2^{1}$ ). Answers of "Middle" are given a minimum value of 0.25 (medium risk, $1 / 2^{2}$ ) and a maximum value of 0.75 (minimal risk). Answers of "Fourth" are given a minimum value of 0.5 and a maximum value of 1.0 (minimal risk with no impact on risk, $1 / 2^{0}$ ). Answers of "Highest" are given a minimum value of 0.75 and a maximum value of 1.0. Maps will range from extreme risk to no risk.

Example Realization Metric Map: Not Available

\section{A.7 Urban characteristics factor overview}

This factor covers characteristics above the household level (neighborhood, city, region) that could contribute to regular social media use. Indicators within this factor include Road Network and Urban Proximity. All indicators currently have available metric data.

\section{A.7.1 Indicator weighting logic}

As both indicators contribute equally to the factor, they are both given a minimum weight value of 0.5 and a maximum weight value of 0.7 .

\begin{tabular}{|l|c|c|}
\hline Indicator & Weight Minimum Value & Weight Maximum Value \\
\hline Road Network & 0.5 & 0.7 \\
\hline Urban Proximity & 0.5 & 0.7 \\
\hline
\end{tabular}

\section{A.7.1.1 Uncertainty values}

This factor is given a range of uncertainty values from 0.2 to 0.4 because of the perceived ability of the existing indicators to fully address the themes of the factor. The addition of indicators accounting for urban infrastructure, as well as qualitative data, could further reduce the uncertainty values.

Factor Uncertainty Min:

Factor Uncertainty Max:
0.2

0.4 


\section{A.7.1.2 Example outcome}

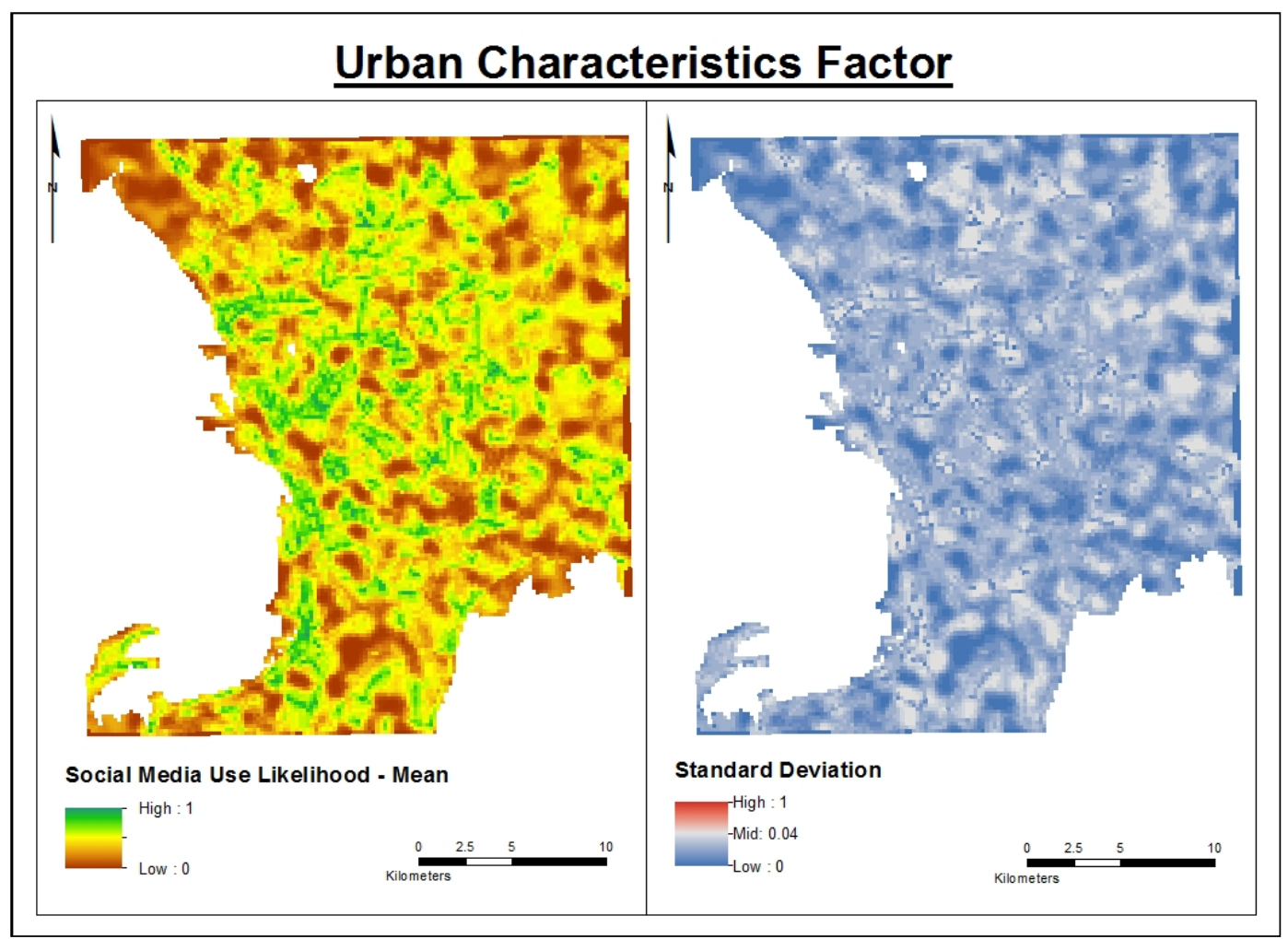

\section{A.7.2 Indicator: Road network}

The Road Network indicator accounts for the proximity of members of the sample population to the road network. Legara found that tweets in the Philippines correlated with population density, GRDP, electricity, and road networks (Legara 2015).

\section{A.7.2.1 Metric weighting logic}

As proximity to major roads is a better indicator than proximity to roads in general, the DistanceToMajorRoad metric is given a higher minimum and maximum value than DistanceToRoad.

Metric

DistanceToRoad

DistanceToMajorRoad
Weight Minimum Value

0.5

0.6
Weight Maximum Value

0.7

0.8 


\section{A.7.2.2 Uncertainty values}

This indicator is given an uncertainty minimum value of 0.1 and an uncertainty maximum value of 0.3 because of the perceived ability of the existing metrics to fully address the themes of the indicator. The addition of transportation data could further reduce the uncertainty values.
Indicator Uncertainty Min:
0.1
Indicator Uncertainty Max:
0.3

\section{A.7.2.3 Example outcome}

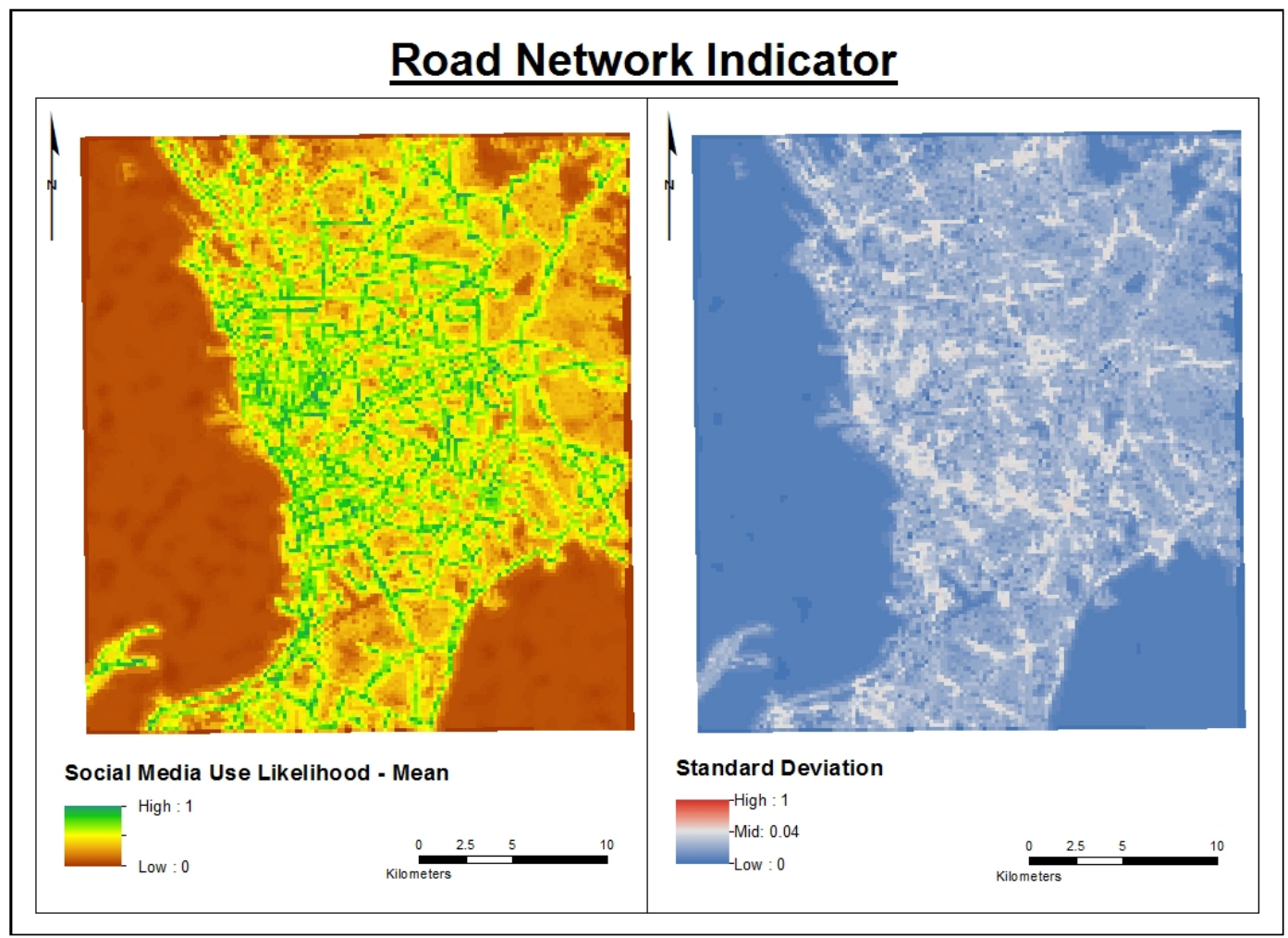

\section{A.7.2.4 Reference}

Legara E. Urbanism in the Philippines. A Byte of my 22-lb Brain. 2015. https://erikafille.ph/2015/09/10/urbanism-in-the-philippines/.

\section{A.7.2.5 Metric: DistanceToRoad}

$\begin{array}{ll}\text { Indicator: } & \text { Road Network } \\ \text { Factor: } & \text { Urban Characteristics } \\ \text { Condition: } & \text { Place } \\ \text { Framework: } & \text { Social Media User }\end{array}$


Metric Assigned Values:
$10 m$ (min: 1.0, max: 1.0)

$20 \mathrm{~m}$ (min: 0.9, max: 0.9)

50m (min: 0.75, max: 0.75)

100m (min: 0.5, max: 0.5)

200m (min: 0.25, max: 0.25)

500m (min: 0.125, max: 0.125)

$>500$ m (min: 0.0625, max: 0.0625)

$\mathrm{N} / \mathrm{A}$

N/A

Other Data Sources: Urban Tactical Planner, Army Geospatial Center

Logic:

This metric consists of distance to all roads in the metro Manila area. The metric map was created in ArcGIS using Multiple Ring Buffer, Polygon to Raster, and Project Raster tools.

Distances of 10 meters or less are given both a minimum and maximum value of 1.0 (minimal risk with no impact on risk, $1 / 2^{0}$ ). Distances between 10 and 20 meters are given both a minimum and maximum value of 0.9 (minimal risk). Distances between 20 and 50 meters are given both a minimum and maximum value of 0.75 (minimal risk). Distances between 50 and 100 meters are given both a minimum and maximum value of 0.5 (slight risk, $1 / 2^{1}$ ). Distances between 100 and 200 meters are given both a minimum and maximum value of 0.25 (medium risk, $1 / 2^{2}$ ). Distances between 200 and 500 meters are given both a minimum and maximum value of 0.125 (high risk, $1 / 2^{3}$ ). Distances greater than 500 meters are given both a minimum and maximum value of 0.0625 (extreme risk, $1 / 2^{4}$ ). Maps will range from extreme risk to no risk. 


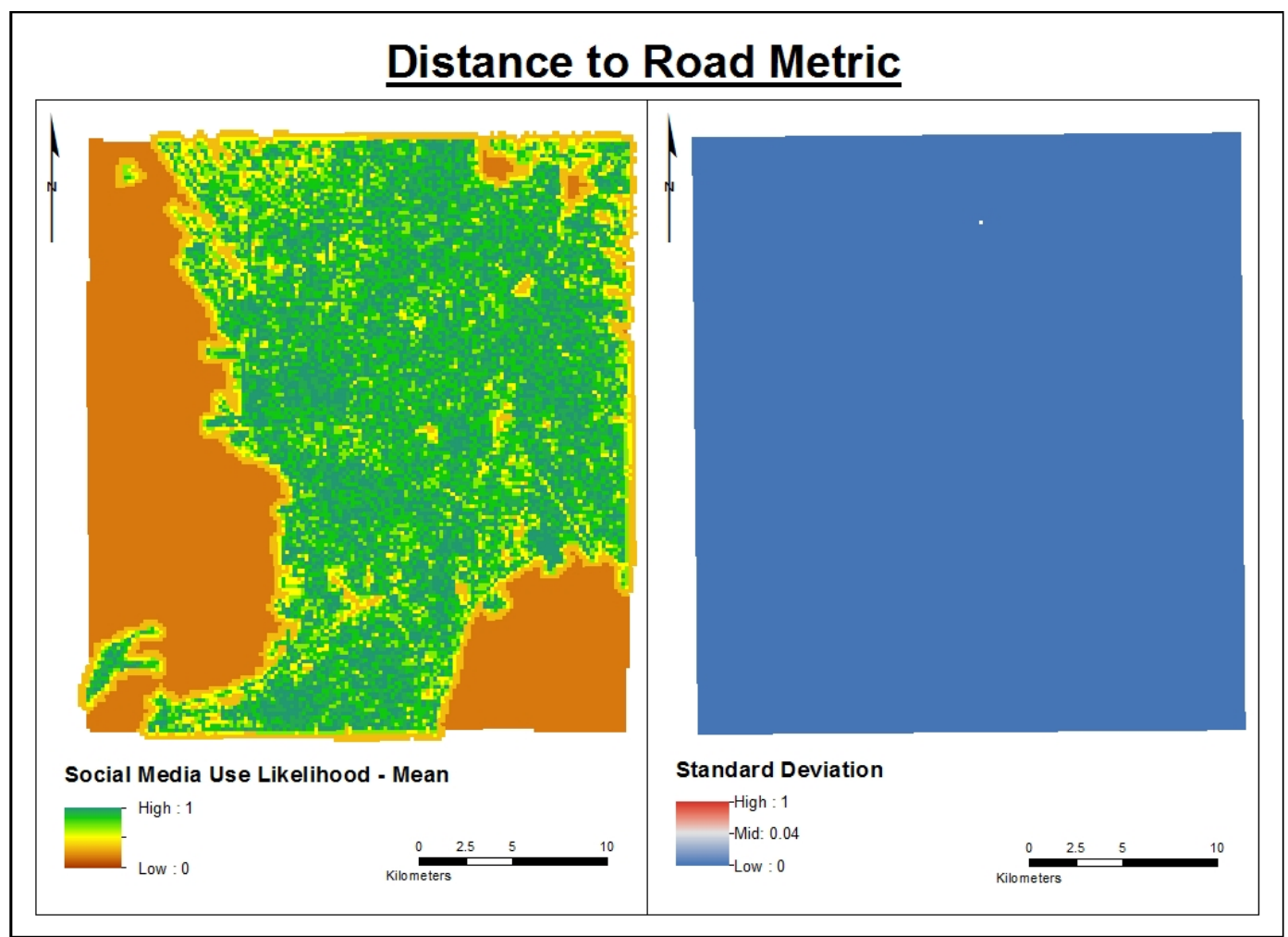

A.7.2.6 Metric: DistanceToMajorRoad

Indicator: $\quad$ Road Network

Factor: Urban Characteristics

Condition: Place

Framework: $\quad$ Social Media User

Metric Assigned $\quad 10 \mathrm{~m}$ (min: 1.0, max: 1.0)

Values:

20m (min: 0.9, max: 0.9)

50m (min: 0.75, max: 0.75)

$100 \mathrm{~m}$ (min: 0.5, max: 0.5)

200m (min: 0.25, max: 0.25)

500m (min: 0.125, max: 0.125)

$>500$ m (min: 0.0625, max: 0.0625)

Survey: $\quad$ N/A

Survey Date: $\quad N / A$

Other Data Urban Tactical Planner, Army Geospatial Center

Sources:

Logic:

This metric consists of distance to major roads, such as freeways and other major thoroughfares. The metric map was created in ArcGIS using Multiple Ring Buffer, Polygon to Raster, and Project Raster tools. Distances of 10 meters or less are given both a minimum and maximum value of 1.0 (minimal risk with no impact on risk, $1 / 2^{0}$ ). Distances between 10 and 20 meters are given both a minimum and 
maximum value of 0.9 (minimal risk). Distances between 20 and 50 meters are given both a minimum and maximum value of 0.75 (minimal risk). Distances between 50 and 100 meters are given both a minimum and maximum value of 0.5 (slight risk, $1 / 2^{1}$ ). Distances between 100 and 200 meters are given both a minimum and maximum value of 0.25 (medium risk, $1 / 2^{2}$ ). Distances between 200 and 500 meters are given both a minimum and maximum value of 0.125 (high risk, $1 / 2^{3}$ ). Distances greater than 500 meters are given both a minimum and maximum value of 0.0625 (extreme risk, $1 / 2^{4}$ ). Maps will range from extreme risk to no risk.

Example Realization Metric Map

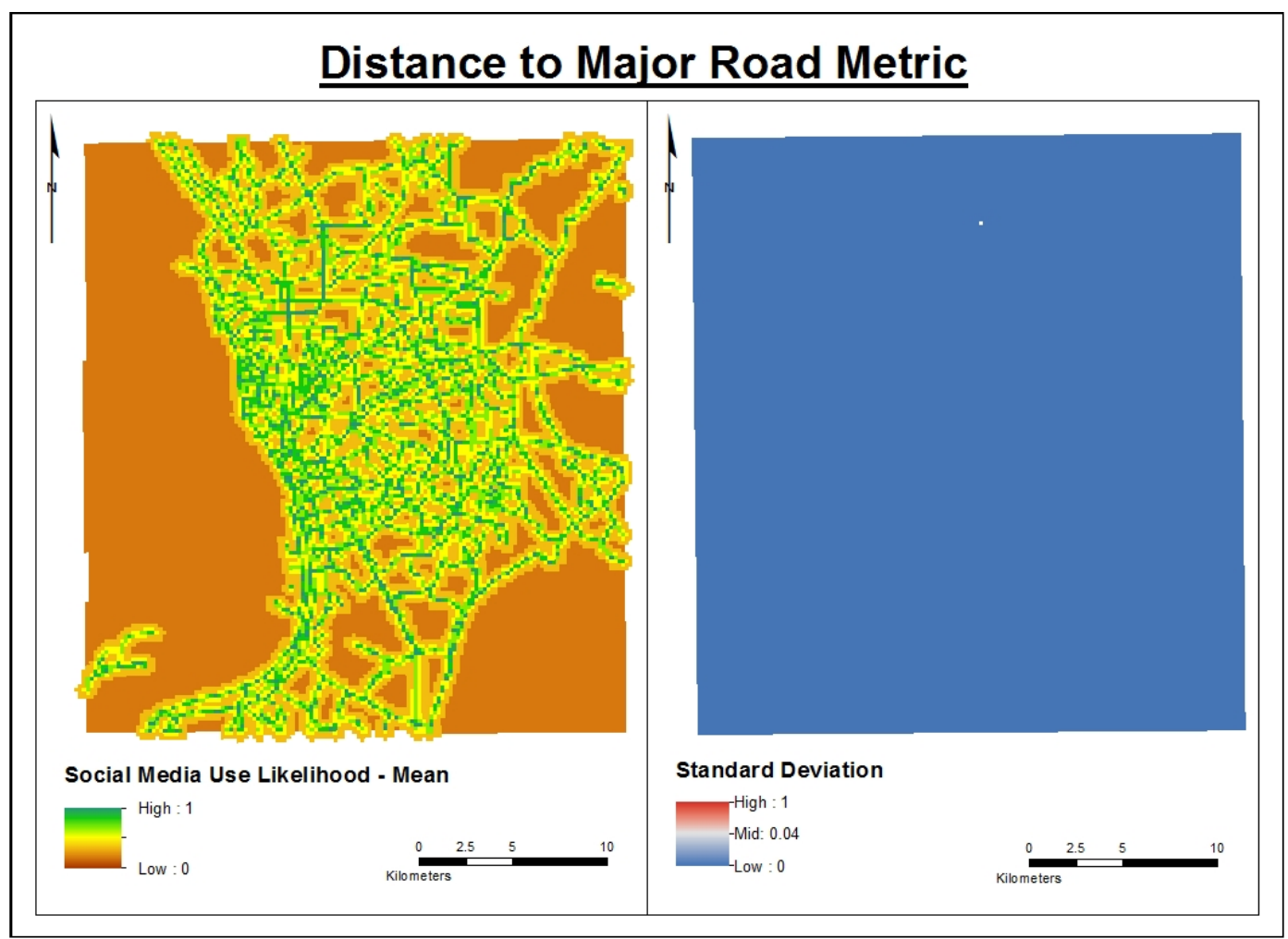

\section{A.7.3 Indicator: Urban proximity}

The Urban Proximity indicator accounts for distance to dense urban areas, as well as proximity to other indicators of urban development.

\section{A.7.3.1 Metric weighting logic}

As there is only one metric in this indicator, it is given both a minimum and maximum value of 1.0.

\section{Metric}

PlaceOfResidence

\section{Weight Minimum Value}

1.0

\section{Weight Maximum Value}

1.0 


\section{A.7.3.2 Uncertainty values}

This indicator is given an uncertainty minimum value of 0.1 and an uncertainty maximum value of 0.3 because of the perceived ability of the existing metrics to fully address the themes of the indicator. The addition of qualitative and land use data could further reduce the uncertainty values.

Indicator Uncertainty Min:

Indicator Uncertainty Max:
0.1

0.3

\section{A.7.3.3 Example outcome}

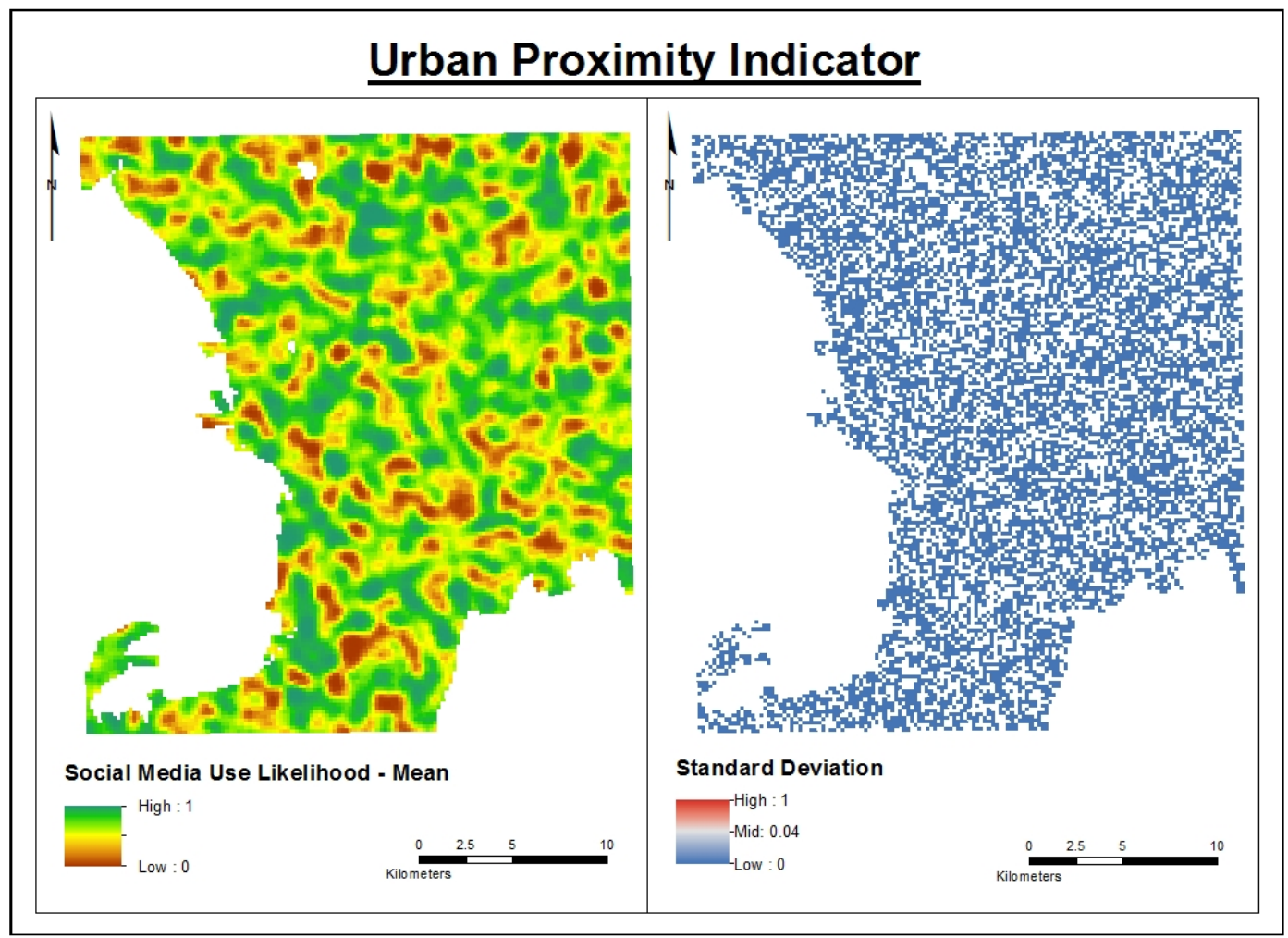

A.7.3.4 Metric: PlaceOfResidence

Indicator: Urban Proximity

Factor: Urban Characteristics

Condition: Place

Framework: Social Media User

Metric Assigned $\quad$ City (min: 0.5, max: 0.9)

Values:

Town Proper/Poblacion (min: 0.25, max: 0.7)

Barrio/Rural Area (min: 0.0625, max: 0.125)

Abroad (min: 0.0, max: 0.0) 
Don't Know (min: 0.0, max: 0.0)

Survey: $\quad$ DHS

Survey Date: $\quad 2013$

Other Data Sources: N/A

Logic: $\quad$ This metric consists of place of residence classifications from the DHS survey. Answers of "City" are given a minimum value of 0.5 (slight risk, $1 / 2^{1}$ ) and a maximum value of 0.9 (minimal risk). Answers of "Town Proper/Poblacion" are given a minimum value of 0.25 (medium risk, $1 / 2^{2}$ ) and a maximum value of 0.7 (minimal risk). Answers of "Barrio/Rural Area" are given a minimum value of 0.0625 (extreme risk, $1 / 2^{4}$ ) and a maximum value of 0.125 (high risk, $1 / 2^{3}$ ). Answers of "Abroad" and "Don't Know" are given both a minimum and maximum value of 0.0 . Maps will range from extreme risk to minimal risk.

Example Realization Metric Map: Not Available

\section{A.7.3.5 References}

Albert, Jose Ramon G., Ramonette B. Serafica and Beverly T. Lumbera. Examining Trends in ICT Statistics: How Does the Philippines Fare in ICT? Philippines Institute for Development Studies. May 2016.

https://dirp3.pids.gov.ph/websitecms/CDN/PUBLICATIONS/pidsdps1616.pdf on 16 Nov 2017.

Andrei, Amanda, Sara Beth Elson, Guido Zarrella. Language and Emotion in Philippine Twitter Use during Typhoon Haiyan. 2016. DOI 10.13140/RG.2.1.4671.5923.

Greenwood, Shannon, Andrew Perrin and Maeve Duggan. Social Media Update 2016. Pew Research Center. Nov 11, 2016. http://www.pewinternet.org/2016/11/11/socialmedia-update-2016/.

Kroulek, Alison. Which Counties Have the Most English Speakers? K International. 27 Feb 2017. http://www.k-international.com/blog/countries-with-the-most-english-speakers/.

Legara E. Urbanism in the Philippines. A Byte of my 22-lb Brain. 2015. https://erikafille.ph/2015/09/10/urbanism-in-the-philippines/.

Third Team Media. The State of Social Media and Digital in the Philippines for 2017. Jan 24, 2017. https://www.slideshare.net/likke13/the-state-of-social-media-and-digital-inthe-philippines-for-2017. 


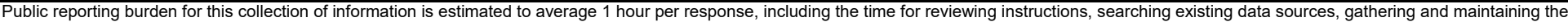

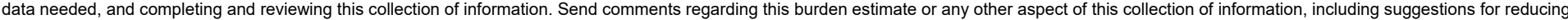

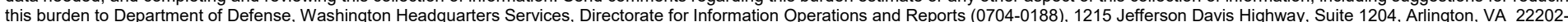

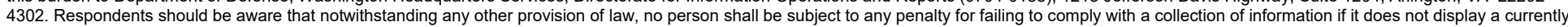
valid OMB control number. PLEASE DO NOT RETURN YOUR FORM TO THE ABOVE ADDRESS.
1. REPORT DATE (DD-MM-YYYY) $09 / 01 / 2019$
Final Technical Report (TR)

\section{TITLE AND SUBTITLE}

Quantifying Uncertainty in Population Weighting of Twitter Analyses for Urban Risk Assessment

Elizabeth G. Bastian, Natalie R. Myers, Charles R. Ehlschlaeger, and Jeffrey A. Burkhalter
3. DATES COVERED (From - To)

5a. CONTRACT NUMBER

5b. GRANT NUMBER

5c. PROGRAM ELEMENT

622784AT41

5d. PROJECT NUMBER

P2 458304

5e. TASK NUMBER

5f. WORK UNIT NUMBER

8. PERFORMING ORGANIZATION REPORT NUMBER

ERDC/CERL TR-19-14

U.S. Army Engineer Research and Development Center (ERDC)

Construction Engineering Research Laboratory (CERL)

PO Box 9005,

Champaign, IL 61826-9005

10. SPONSOR/MONITOR'S ACRONYM(S) ASA(ALT)

Office of the Assistant Secretary of the Army for Acquisition, Logistics, and Technology (ASA[ALT]) 3010 Defense Pentagon

Washington, DC 20301-3010

11. SPONSOR/MONITOR'S REPORT NUMBER(S)

\section{DISTRIBUTION / AVAILABILITY STATEMENT}

Approved for public release; distribution is unlimited.

\section{SUPPLEMENTARY NOTES}

\section{ABSTRACT}

Twitter has increasingly been used to study various research topics such as election predictions, disease spread, etc. However, social media platforms do not saturate the entire population in a study area, especially in emerging nations, only representing more affluent subpopulations. The U.S. Army Engineer Research and Development Center, Construction Engineering Research Laboratory (ERDCCERL), as part of a project entitled Framework for the Integration of Complex Urban Systems (FICUS), is quantifying the utility of demographic information to inform neighborhood-scale social media models. Using the example topic of infrastructure, an opensource model was constructed to collect Twitter data from the metropolitan Philippines area of Manila, geotag tweets to neighborhood grid cells based on language analysis, and produce a sentiment topic map. ERDC's social media analysis tools incorporate quantifiable uncertainties with specific on-the-ground reporting techniques. By using the Humanitarian Crisis (HC) framework developed by PACOM (another FICUS product) as a model, a framework quantifying the likelihood of being a regular social media user was created to implement a data-driven, bottom-up framework construction nested within a knowledge-based established framework. This framework, and any other produced by the FICUS team serve as case studies for augmenting the military operational environment with quantifiable reduced uncertainties.

\section{SUBJECT TERMS}

Social media, Social media-Analysis, Twitter, Population-Analysis, Military operations

\section{SECURITY CLASSIFICATION OF:}

\section{a. REPORT}

Unclassified

\section{b. ABSTRACT \\ Unclassified}

17. LIMITATION OF ABSTRACT

\section{c. THIS PAGE}

Unclassified
SAR

\begin{tabular}{|c|c|}
$\begin{array}{l}\text { 18. NUMBER } \\
\text { OF PAGES }\end{array}$ & 19 \\
\cline { 2 - 2 } & \\
\cline { 2 - 2 } &
\end{tabular}

19a. NAME OF RESPONSIBLE PERSON

19b. TELEPHONE NUMBER

(include area code) 